\title{
THE METRIC ANOMALY OF ANALYTIC TORSION ON MANIFOLDS WITH CONICAL SINGULARITIES
}

\author{
WERNER MÜLLER AND BORIS VERTMAN
}

\begin{abstract}
In this paper we study the analytic torsion of an odd-dimensional manifold with isolated conical singularities. First we show that the analytic torsion is invariant under deformations of the metric which are of higher order near the singularities. Then we identify the metric anomaly of analytic torsion for a bounded generalized cone at its regular boundary in terms of spectral information of the crosssection. In view of previous computations of analytic torsion on cones, this leads to a detailed geometric identification of the topological and spectral contributions to analytic torsion, arising from the conical singularity. The contribution exhibits a torsion-like spectral invariant of the cross-section of the cone, which we study under scaling of the metric on the cross-section.
\end{abstract}

\section{Contents}

1. Introduction 2 Acknowledgments 3

2. Preliminaries and statement of main results 3

2.1. Definition and variation of Analytic Torsion 3

2.2. Analytic Torsion of a bounded Cone 7

3. Variation of Analytic Torsion under Higher Order Deformations 9

3.1. The Heat Kernel on Manifolds with Conical Singularities 10

3.2. Analytic Torsion under Metric Variation at the Cone Tip 12

4. Scaling Invariance of the Metric Anomaly for Analytic Torsion 17

5. Analytic Torsion of the Truncated Cone 18

5.1. Decomposition of the de Rham Complex of the Truncated Cone 18

5.2. The Relative Ideal Boundary Conditions 21

5.3. The Difference of Analytic Torsion for the Truncated and the Full Cone 23

6. Metric Anomaly at the Regular Boundary of the Cone 40

7. Asymptotics of the New Torsion-Like Spectral Invariant 43

References $\quad 46$

$\begin{array}{ll}\text { List of Figures } & 47\end{array}$

Date: September 24, 2018.

2000 Mathematics Subject Classification. 58J52; 34B24.

Both authors were partially supported by the Hausdorff Center for Mathematics. 


\section{INTRODUCTION}

In this paper we study the Ray-Singer analytic torsion of a compact odd-dimensional Riemannian manifold with an isolated conical singularity. This means the following. We consider an open Riemannian manifold $\left(M, g^{M}\right)$ of odd dimension which admits a decomposition

$$
M=\mathscr{U} \cup_{N} X,
$$

into a compact Riemannian manifold $X$ with boundary $N$ and an open truncated generalized cone $\mathscr{U}$ over $N$. Here $\mathscr{U}=(0,1] \times N$, and the metric on $\mathscr{U}$ takes the special form

$$
\left.g^{M}\right|_{\mathscr{U}}:=d x^{2} \oplus x^{2} g^{N}(x), x \in(0,1),
$$

where $g^{N}(x)$ is a family of metrics on $N$ which is smooth up to 0 . Our goal is to study the analytic torsion of such manifolds. We mention that the case of an even-dimensional cone has been studied by the second named author [VER11] and Hartmann-Spreafico [HASp10]. This case is simpler than the odd-dimensional case.

The analytic torsion of a compact Riemannian manifold, equipped with a flat Hermitian vector bundle, was introduced by Ray and Singer [RASr71] as an analytic counterpart of the Reidemeister-Franz torsion. The latter is defined combinatorial, whereas the analytic torsion is defined as a weighted product of regularized determinants of the Laplacian on $p$-forms with values in the flat bundle. The conjecture of Ray and Singer that the two invariants are equal was proved independently by Cheeger [CHE79A] and Müller [MüL78]. Lück [LÜC93] and Vishik [VIS95] extended this result to compact Riemannian manifolds with boundary under the assumption that the metric is a product near the boundary. Equality still holds up to a term which is given by the Euler characteristic of the boundary. If the metric is not a product near the boundary, additional terms, called anomaly, arise in the difference between the analytic and the combinatorial torsion (see [DAFA00] and [BRMA06]).

A natural problem is to determine whether the equality of analytic torsion and Reidemeister torsion for compact Riemannian manifolds has any analogue for manifolds with singularities. To begin with one needs to define both invariants for such spaces. On the combinatorial site, Dar [DAR87] has defined the intersection R-torsion for pseudomanifolds. Furthermore he has shown that the analytic torsion is well-defined for compact manifolds with conical singularities. Therefore it makes sense to study the relation between analytic torsion and intersection torsion for compact manifolds with conical singularities. The present paper is a contribution to this goal. As already mentioned, we study the analytic torsion of a manifold with conical singularities.

First we derive a variational formula which shows that we may assume that the metric in a neighborhood of the cone tip is an exact cone metric. Using a splitting formula (which we don't prove in this paper) together with [BRMa06], it follows that one can replace $M$ by an exact cone $C(N)$, i.e., $C(N)=(0,1] \times N$, equipped with the metric $d x^{2}+x^{2} g^{N}(0)$, and study the problem for this case.

In [Ver09A] the second named author has derived a formula which expresses the analytic torsion of $C(N)$ in terms of spectral invariants of $N$. In the present paper we will identify some of these terms. More precisely we will show that the analytic 
torsion of $C(N)$ is the sum of three terms. The first one is a linear combination of Betti numbers. The second term equals the metric anomaly of Brüning and Ma [BRMA06]. The remaining term is a torsion-like spectral invariant of the cross section $N$.

The paper is organized as follows. In Section 2 we will summarize the basic facts about analytic torsion of manifolds with conical singularities and state the main results. In Section 3 we will establish invariance of the analytic torsion under higher order deformations of the conical metric, assuming essential self-adjointness of the GaußBonnet operator. The result is a special case of a more general investigation of analytic torsion on manifolds with incomplete edge singularities by Mazzeo and the second author in [MAVE10]. Essential self-adjointness of the Gauß-Bonnet operator is posed only within the limits of Section 3 and can always be achieved by appropriate rescaling of $g^{N}(0)$.

In Section 4 we discuss the metric anomaly formula for Ray-Singer analytic torsion on manifolds with boundary, established by Brüning-Ma in [BRMA06]. Especially we prove that the metric anomaly is scaling invariant.

We proceed in Section 5 with computing analytic torsion of a truncated cone. This leads in Section 6 to an explicit identification of the metric anomaly at the regular boundary of the cone in terms of the spectral information of the cross-section $N$. This shows that the main contribution of the conical singularity to the analytic torsion is given by a torsion-like spectral invariant of the cross section.

In Section 7 we study the behavior of this invariant under scaling of the metric on the base of the cone and compute its value for some examples.

\section{ACKNOWLEDGMENTS}

It is a pleasure for the second author to acknowledge the helpful discussions with Rafe Mazzeo and Jeff Cheeger. Furthermore both authors would like to thank Jochen Brüning and Xiaonan Ma for explaining important aspects of the metric anomaly for analytic torsion on manifolds with boundary, as well as Matthias Lesch. The second author would like to express his gratitude to Stanford University where the research has partially been conducted for hospitality, and Hausdorff Center for Mathematics in Bonn.

\section{Preliminaries and Statement of main Results}

In this section we summarize some facts about the analytic torsion for manifolds with conical singularities and we state the main results.

2.1. Definition and variation of Analytic Torsion. Let $\left(M^{m}, g^{M}\right)$ be an odddimensional oriented Riemannian manifold with an isolated conical singularity. Thus $M=\mathscr{U} \cup_{N} X$, where $\left(X,\left.g\right|_{X}\right)$ is a compact Riemannian manifold with boundary $\partial X=N$ and $\mathscr{U}$ is a truncated generalized cone, which means that $\mathscr{U}=(0,1) \times N$ and the metric on $\mathscr{U}$ is given by

$$
\left.g^{M}\right|_{\mathscr{U}}:=d x^{2} \oplus x^{2} g^{N}(x),
$$


where $g^{N} \in C^{\infty}\left([0,1], \operatorname{Sym}^{2}\left(T^{*} N\right)\right)$. Actually the assumptions on the the behavior of $g^{N}(x)$ at $x=0$ can be weakened (see [BRLE93, Section 2]). For simplicity we will work with the stronger assumption that $g^{N}(x)$ is smooth up to 0 .

Fix a base point $x_{0} \in M$ and let

$$
\rho: \pi_{1}\left(M, x_{0}\right) \rightarrow U(n, \mathbb{C})
$$

be a unitary representation of the fundamental group $\pi_{1}\left(M, x_{0}\right)$. Let $\left(E, \nabla, h^{E}\right)$ be the flat Hermitian vector bundle over $M$, associated to the representation $\rho$. Here $h^{E}$ is the flat Hermitian metric on $E$, induced by the standard Hermitian inner product on $\mathbb{C}^{n}$. We may assume that $x_{0}=\left(t, y_{0}\right) \in \mathscr{U}$. Then $\left.E\right|_{\mathscr{U}}$ is the flat bundle associated to the composition $\rho_{N}$ of $\pi_{1}\left(\mathscr{U}, x_{0}\right) \rightarrow \pi_{1}\left(M, x_{0}\right)$ and $\rho$. Note that $\pi_{1}\left(N, y_{0}\right) \cong \pi_{1}\left(\mathscr{U}, x_{0}\right)$. So we may regard $\rho_{N}$ as a representation of $\pi_{1}\left(N, y_{0}\right)$. Let $\left(E_{N}, \nabla_{N}, h_{N}\right)$ be the associated flat Hermitian bundle. Then we have

$$
\left.E\right|_{\mathscr{U}} \cong p^{*}\left(E_{N}\right) \cong(0,1] \times E_{N},
$$

where $p:(0,1] \times N \rightarrow N$ is the canonical projection. Moreover the isomorphism (2.3) is compatible with the Hermitian metrics and the flat connections. The flat covariant derivatives $\nabla$ and $\nabla_{N}$ are related as follows. For $s \in C^{\infty}\left([0,1], C^{\infty}\left(E_{N}\right)\right) \cong \Gamma\left(\left.E\right|_{\mathscr{U}}\right)$ we have

$$
\nabla s=\frac{\partial s}{\partial x} \otimes d x+\nabla_{N} s
$$

Let $\left(\Omega^{*}(M, E), d_{*}\right)$ be the associated twisted de Rham complex, where $\Omega^{*}(M, E)$ denotes the space of $E$-valued differential forms. Denote by $\Omega_{c}^{*}(M, E)$ the subspace of differential forms with compact support. Let $\Delta_{k}$ be the corresponding Laplace operator on $k$-forms. We regard $d_{k}$ and $\Delta_{k}$ as unbounded operators in $L^{2} \Omega^{k}(M, E)$ with domain $\Omega_{c}^{k}(M, E)$.

Consider the maximal extension $d_{k, \max }$ of $d_{k}$ in $L^{2} \Omega^{k}(M, E)$ with domain $\mathscr{D}\left(d_{k, \max }\right)$. The minimal extension $d_{k \text {,min }}$ of $d_{k}$ with domain $\mathscr{D}\left(d_{k, \text { min }}\right) \subset \mathscr{D}\left(d_{k, \text { max }}\right)$ is the graph closure of $d_{k}$. Ideal boundary conditions for the de Rham complex $\left(\Omega^{*}(M, E), d_{*}\right)$ is a choice of closed extensions $D_{k}$ of $d_{k}$ for each $k=0, \ldots, \operatorname{dim} M$ with

$$
\mathscr{D}\left(d_{k, \min }\right) \subseteq \mathscr{D}\left(D_{k}\right) \subseteq \mathscr{D}\left(d_{k, \text { max }}\right),
$$

which combine into a Hilbert complex in the sense of [BRLE93]. Ideal boundary conditions for the de Rham complex induce a self-adjoint extension for each $\Delta_{k}$. The two special cases are the relative and absolute boundary conditions

$$
\begin{aligned}
\Delta_{k, \text { rel }} & =d_{k, \text { min }}^{*} d_{k, \text { min }}+d_{k-1, \text { min }} d_{k-1, \text { min }}^{*} \\
\Delta_{k, \text { abs }} & =d_{k, \text { max }}^{*} d_{k, \text { max }}+d_{k-1, \text { max }} d_{k-1, \text { max }}^{*} .
\end{aligned}
$$

We recall from [CHE83] and [BRLE93, Theorem 3.8] the following facts. Ideal boundary conditions are unique in degrees $k \neq(m-1) / 2, m=\operatorname{dim} M$. If we assume the Wittcondition $H^{p}\left(N, E_{N}\right)=0, p=(m-1) / 2$, then ideal boundary conditions are unique also in degree $p$.

However, the Witt condition which implies uniqueness of the ideal boundary conditions, does not imply essential self-adjointness of $\Delta_{k}$ and the Laplacian corresponding to the (unique) ideal boundary conditions need not be given by the Frederich's extension in degree $k=(m \pm 1) / 2$. In fact, by [BRLE93, Corollary 3.5] the extensions 
$\Delta_{k \text {,rel }}$ and $\Delta_{k \text {,abs }}$ are given by the Friedrich extension in any degree if and only if the Gauß-Bonnet operator is essentially self-adjoit. This can always be achieved by scaling $g^{N}(0)$ to $c^{2} g^{N}(0)$ with $c>0$ sufficiently small.

Choose either the relative or the absolute self-adjoint extension of $\Delta_{k}$, which we denote again by the same letter for the moment. It is known that $\Delta_{k}$ has pure point spectrum [Les97] and by [Les97, Corollary 2.4.3] it follows that for every $t>0$, $\exp \left(-t \Delta_{k}\right)$ is a trace class operator. Moreover we have the following theorem.

Theorem 2.1. Let $(M, g)$ be a compact odd-dimensional Riemannian manifold of dimension $m$ with a conical singularity. Let $\left(E, \nabla, h^{E}\right)$ be a flat Hermitian vector bundle over $M$ and $\left(E_{N}, \nabla_{N}, h_{N}\right)$ the associated bundle over $N$. If $g^{N}(x)$ is not constant in $x \in[0,1]$, we assume essential self-adjointness of the Gauß-Bonnet operator. Then for the absolute or relative self-adjoint extension $\Delta_{k}$ of the Laplacian on $\Omega_{c}^{k}(M, E)$, $k=0, \ldots, m$, we have

$$
\operatorname{Tr}\left(e^{-t \Delta_{k}}\right) \sim_{t \rightarrow 0+} \sum_{j=0}^{\infty} A_{k} t^{j-\frac{m}{2}}+\sum_{j=0}^{\infty} C_{j} t^{\frac{j}{2}}+\sum_{j=1}^{\infty} G_{j} t^{\frac{j}{2}} \log t .
$$

For $g^{N} \equiv g^{N}(0)$ the result is well-known (see [CHE83]). Otherwise, asymptotic expansions with $G_{0}=0$ are known only for the Frederich's extension $\Delta^{\mathscr{F}}$ (see [Moo99]) and essential self-adjointness of the Gauß-Bonnet operator is imposed above to guarantee $\Delta=\Delta^{\mathscr{F}}$.

Remark 2.2. In fact it suffices to impose the Witt condition $H^{p}\left(N, E_{N}\right)=0, p=$ $(m-1) / 2$ instead of essential self-adjointness of the Gauß-Bonnet operator for Theorem 2.1 to hold in case $g^{N}(x)$ is not constant in $x \in[0,1]$. Under the Witt condition the heat kernel of $\Delta_{k}$ is still polyhomogeneous on a certain parabolic blowup of $\mathbb{R}^{+} \times M^{2}$ (see [Moo99]) and the heat trace expansion follows by the Push-forward theorem. We confine ourselves to the case of essentially self-adjoint Gauß-Bonnet operator for simplicity.

Let $\lambda_{0} \leq \lambda_{1} \leq \cdots$ denote the eigenvalues of $\Delta_{k}$. It follows from (2.6) that Weyl's law holds for the counting function of the eigenvalues. This implies that the zeta function

$$
\zeta\left(s ; \Delta_{k}\right):=\sum_{\lambda_{j}>0} \lambda_{j}^{-s}
$$

converges in the half-plane $\operatorname{Re}(s)>m / 2$ and it can be expressed in terms of the trace of the heat operator by

$$
\zeta\left(s, \Delta_{k}\right)=\frac{1}{\Gamma(s)} \int_{0}^{\infty} t^{s-1} \operatorname{Tr}\left(e^{-t \Delta_{k}}-P_{k}\right) d t, \quad \operatorname{Re}(s)>m / 2,
$$

where $P_{k}$ denotes the orthogonal projection of $L^{2} \Omega^{k}(M, E)$ onto the subspace $\mathscr{H}_{(2)}^{k}(M, E)$ of harmonic $k$-forms. Then the asymptotic expansion (2.6) yields the meromorphic extension of the right hand side and hence, of the zeta function, to the whole complex plane. Furthermore it also follows from $(2.6)$ that $\zeta\left(s, \Delta_{k}\right)$ is regular at $s=0$. We can now define the analytic torsion of $\left(M, g^{M}\right)$ and $\left(E, \nabla, h^{E}\right)$ as in the compact case. Let $\Delta_{k \text {,abs }}$ (resp. $\Delta_{k \text {,rel }}$ ) denote the self-adjoint extension of $\Delta_{k}$ with respect 
to the choice of absolute (resp. relative) boundary conditions. Recall that boundary conditions need to be imposed only if $k=(m \pm 1) / 2$. Then we define

$$
\log T_{\mathrm{abs}}\left(M, E ; g^{M}\right):=\left.\frac{1}{2} \sum_{k=0}^{m}(-1)^{k} \cdot k \cdot \frac{d}{d s} \zeta\left(s, \Delta_{k, \mathrm{abs}}\right)\right|_{s=0},
$$

and define $T_{\text {rel }}\left(M, E ; g^{M}\right)$ similarly. Since $h^{E}$ is the canonical metric on $E$, induced by the unitary representation, we suppress it from the notation. Observe that $* \Delta_{k, \text { abs }}=\Delta_{m-k \text {,rel }} *$. Denote the Laplacians on closed or coclosed differential forms by an additional subscript $c l$ or $c c l$, respectively.

Since the dimension of $M$ is odd, it follows by Poincaré duality that

$$
\begin{aligned}
T_{\mathrm{abs}}\left(M, E ; g^{M}\right) & =\left.\sum_{k=0}^{m-1}(-1)^{k+1} \frac{d}{d s} \zeta\left(s, \Delta_{k, c c l, \mathrm{abs}}\right)\right|_{s=0} \\
& =\left.\sum_{k=0}^{m-1}(-1)^{k+1} \frac{d}{d s} \zeta\left(s, \Delta_{m-k, c l, \mathrm{rel}}\right)\right|_{s=0} \\
& =\left.\sum_{k=0}^{m-1}(-1)^{k+1} \frac{d}{d s} \zeta\left(s, \Delta_{k, c c l, \text { rel }}\right)\right|_{s=0}=T_{\text {rel }}\left(M, E ; g^{M}\right) .
\end{aligned}
$$

For the second equality we have used that $* \Delta_{k, c c l, \text { abs }}=\Delta_{m-k, c l, \text { rel }} *$. The third equality follows from the fact that for an eigenvalue $\lambda \neq 0, d$ is an isomorphism between the $\lambda$ eigenspace of the Laplacian on co-closed forms, satisfying relative boundary conditions, and the $\lambda$-eigenspace of the Laplacian on closed forms, satisfying relative boundary conditions. So we will denote the torsion simply by $T\left(M, E ; g^{M}\right)$.

Remark 2.3. Dar [DAR87] also discusses the analytic torsion of a manifold with conical singularities, where the cross section $N$ is allowed to have a non-empty boundary. In this case the zeta-functions may have a pole at $s=0$. However, due to residue cancellations, the analytic torsion is still well-defined. If $\partial N=\emptyset$, the case treated in the present paper, the zeta-functions are regular at zero individually.

For a finite-dimensional vector space $F$, set $\operatorname{det} F:=\Lambda^{\max } F$ and $\operatorname{denote}$ by $(\operatorname{det} F)^{-1}$ the dual line $\operatorname{det} F^{*}$. Let $\mathscr{H}_{(2)}^{*}(M, E)$ be the space of square integrable harmonic forms $\mathscr{H}_{(2)}^{*}(M, E)$ which satisfy absolute boundary conditions. The determinant line of $(M, E)$ is defined as

$$
\operatorname{det} \mathscr{H}_{(2)}^{*}(M, E):=\bigotimes_{k=0}^{m}\left[\operatorname{det} \mathscr{H}_{(2)}^{k}(M, E)\right]^{(-1)^{k+1}} .
$$

Definition 2.4. The Ray-Singer metric $\|\cdot\|_{\left(M, E ; g^{M}\right)}^{R S}$ on $\operatorname{det} \mathscr{H}_{(2)}^{k}(M, E)$ is defined by

$$
\|\cdot\|_{\left(M, E ; g^{M}\right)}^{R S}:=T\left(M, E ; g^{M}\right) \cdot\|\cdot\|_{\operatorname{det} \mathscr{H}_{(2)}^{k}(M, E)},
$$

where $\|\cdot\|_{\operatorname{det} \mathscr{H}_{(2)}^{k}(M, E)}$ is the norm on $\operatorname{det} \mathscr{H}_{(2)}^{k}(M, E)$ induced by the $L^{2}$-norm on the space of harmonic forms $\mathscr{H}_{(2)}^{*}(M, E)$. 
Using methods developed by Melrose [MeL93], we study the variation of the analytic torsion with respect to the variation of the metric $g^{M}$. Our main result is the invariance of analytic torsion of manifolds with isolated conical singularities under higher order deformations of the metric near the conical tip, assuming essential self-adjointness of the Gauß-Bonnet operator. More precisely, we have

Theorem 2.5. Let $\left(M^{m}, g^{M}\right)$ be a compact odd-dimensional Riemannian manifold with an isolated conical singularity, and a neighborhood $\mathscr{U} \cong(0,1) \times N$ of the singularity with

$$
g^{M} \uparrow \mathscr{U}=d x^{2} \oplus x^{2} g^{N}(x), g^{N} \in C^{\infty}\left([0,1], \operatorname{Sym}^{2}\left(T^{*} N\right)\right) .
$$

Let $\left(E, \nabla, h^{E}\right)$ be a flat Hermitian vector bundle and $\left(E_{N}, \nabla_{N}, h_{N}\right)$ its restriction over $N$. Assume essential self-adjointness of the Gauß-Bonnet operator. Then the RaySinger metric $\|\cdot\|_{\left(M, E ; g^{M}\right)}^{R S}$ is invariant under all deformations of $g^{M}$ that fix $g^{N}(0)$.

2.2. Analytic Torsion of a bounded Cone. It follows from Theorem 2.5 that for spaces with isolated conical singularities with appropriately rescaled $g^{N} \equiv g^{N}(0)$, so that the Gauß-Bonnet operator is essentially self-adjoint, we may assume that the neighborhood of the singularity is a bounded exact cone $C(N)$, i.e.,

$$
C(N)=(0,1] \times N, \quad g=d x^{2}+x^{2} g^{N}, g^{N} \in \operatorname{Sym}^{2}\left(T^{*} N\right) .
$$

Now recall that for a compact manifold there is a splitting formula for the analytic torsion [VIS95]. It is very likely that the splitting formula continuous to hold for compact manifolds with conical singularities. Assuming this, the study of the analytic torsion for such manifolds can be reduced to the study of analytic torsion of a bounded cone. In [VER09A], the second author has derived a formula which expresses the analytic torsion of a bounded cone in terms of spectral and topological data of the cross section. The computation uses the double-summation method developed by Spreafico in [SpR05], [SpR06], and a symmetry observation by Lesch [LES94]. We recall the result in odd dimensions.

Theorem 2.6. (Vertman, [VER09A]) Let $(C(N), g)$ be a bounded cone over a closed even-dimensional oriented Riemannian manifold $\left(N^{n}, g^{N}\right)$. Let $\left(E, \nabla, h^{E}\right)$ be a flat Hermitian vector bundle over $C(N)$ and $\left(E_{N}, \nabla_{N}, h_{N}\right)$ the associated flat Hermitian vector bundle over $N$. Let $b_{k}=\operatorname{dim} H^{k}\left(N, E_{N}\right), k=0, \ldots, n$, and $\chi\left(N, E_{N}\right)$ the Euler characteristic of $\left(N, E_{N}\right)$. Denote by $\Delta_{k, c c l, N}$ the Laplacian on coclosed $E_{N}$-valued $k$ forms on $N$ and define

$$
\begin{aligned}
& \alpha_{k}:=\frac{(n-1)}{2}-k, \quad \nu(\eta)=\sqrt{\eta+\alpha_{k}^{2}}, \text { for } \eta \in E_{k}=\operatorname{Spec} \Delta_{k, c c l, N} \backslash\{0\}, \\
& \zeta_{k, N}(s)=\sum_{\eta \in E_{k}} \mathrm{~m}(\eta) \nu(\eta)^{-s}, \quad \zeta_{k, N}\left(s, \pm \alpha_{k}\right):=\sum_{\eta \in E_{k}} \mathrm{~m}(\eta)\left(\nu(\eta) \pm \alpha_{k}\right)^{-s}, \quad \operatorname{Re}(s) \gg 0
\end{aligned}
$$

where $\mathrm{m}(\eta)$ denotes the multiplicity of $\eta \in E_{k}$. Then the logarithm of the analytic torsion of $C(N)$ is given by a sum of three terms:

$$
\log T(C(N), E ; g)=\operatorname{Top}\left(N, E_{N} ; g^{N}\right)+\operatorname{Tors}\left(N, E_{N} ; g^{N}\right)+\operatorname{Res}\left(N, E_{N} ; g^{N}\right)
$$


where the first term is a combination of Betti numbers

$$
\begin{aligned}
\operatorname{Top}\left(N, E_{N} ; g^{N}\right)= & \frac{\log 2}{2} \chi\left(N, E_{N}\right) \\
& -\sum_{k=0}^{\frac{n}{2}-1}(-1)^{k} b_{k}\left(\frac{1}{2} \log (n-2 k+1)+\sum_{l=0}^{\frac{n}{2}-k-1} \log (2 l+1)\right) .
\end{aligned}
$$

The second term, which is a torsion-like term, is given by

$$
\operatorname{Tors}\left(N, E_{N} ; g^{N}\right)=\frac{1}{2} \sum_{k=0}^{n-1}(-1)^{k} \zeta_{k, N}^{\prime}\left(0, \alpha_{k}\right)
$$

The third term, the residual term, is a combination of residues of $\zeta_{k, N}(s)$ :

$$
\operatorname{Res}\left(N, E_{N} ; g^{N}\right)=\sum_{k=0}^{n / 2-1} \frac{(-1)^{k}}{4} \sum_{r=1}^{n / 2} \operatorname{Res}_{s=2 r} \zeta_{k, N}(s) \sum_{b=0}^{2 r} A_{r, b}\left(\alpha_{k}\right) \frac{\Gamma^{\prime}(b+r)}{\Gamma(b+r)},
$$

where the coefficients $A_{r, b}\left(\alpha_{k}\right)$ are determined by certain recursive formulas, associated to combinations of special functions.

Remark 2.7. The computations in [VER09A] are only done in the case of the trivial bundle $E$. However, using (2.3), everything in [VER09A] can be extended to the twisted case without difficulties.

Our next result identifies $\operatorname{Res}\left(N, E_{N} ; g^{N}\right)$ with the metric anomaly of the boundary $\{1\} \times N$ of $(C(N), g)$. The metric anomaly has been studied in [BRMA06]. We recall the basic facts. Let $\left(X, g^{X}\right)$ be an oriented compact Riemannian manifold of odd dimensions, with boundary $\partial X$. Let $\left(E, \nabla^{E}, h^{E}\right)$ be flat Hermitian vector bundle over $X$. Let $\nabla^{T X}$ be the Levi-Civita connection associated to $g^{X}$. Brüning and Ma define a secondary class $B\left(\nabla^{T X}\right) \in \Omega^{*}\left(\partial X,\left.E\right|_{\partial X}\right)$ (see [BRMA06, (1.19)]) which describes the metric anomaly in the following sense. Let $g_{i}^{X}, i=1,2$, be two Riemannian metrics on $X$ and let $\nabla_{i}^{T X}$ denote the corresponding Levi-Civita connections. Denote by $\|\cdot\|_{\left(X, E ; g_{i}^{X}\right)}^{R S}$, $i=1,2$ the Ray-Singer metrics on $\operatorname{det} H^{*}(X, E)$. Then

$$
\log \left(\frac{\|\cdot\|_{\left(X, E ; g_{1}^{X}\right)}^{R S}}{\|\cdot\|_{\left(X, E ; g_{2}^{X}\right)}^{R S}}\right)=\frac{\operatorname{rank}(E)}{2}\left[\int_{\partial X} B\left(\nabla_{2}^{T X}\right)-\int_{\partial X} B\left(\nabla_{1}^{T X}\right)\right] .
$$

Now we can state our next result.

Theorem 2.8. Let $(C(N), g)$ be the cone over a closed oriented Riemannian manifold $\left(N^{n}, g^{N}\right)$ of even dimension. Let $\left(E, \nabla, h^{E}\right)$ be a flat Hermitian vector bundle over $C(N)$ and $\left(E_{N}, \nabla_{N}, h_{N}\right)$ the associated flat vector bundle over $N$. Let $\nabla^{T C(N)}$ be the Levi-Civita connection of $g$. Then the residual term of Theorem 2.6 equals

$$
\operatorname{Res}\left(N, E_{N} ; g^{N}\right)=-\frac{\operatorname{rank}(E)}{2} \int_{N} B\left(\nabla^{T C(N)}\right),
$$

where $N$ is identified with $\{1\} \times N$. 
We note that De Melo, Hartmann and Spreafico [HASP10] and [DMHS09] computed the analytic torsion in the special case of a cone over the sphere $S^{n}$ and verified that in even dimensions the residual term $\operatorname{Res}\left(N, E_{N} ; g^{N}\right)$ equals the anomaly term by direct comparison.

Remark 2.9. Consider the example of a cone over the $n$-dimensional flat torus $T^{n}$ and assume that $E$ is the trivial line bundle. Then from (2.15) one finds that the integral of $B\left(\nabla^{T C\left(T^{n}\right)}\right)$ is given in terms of residues of the shifted zeta function $\zeta_{k, N}(s)$. These are rational multiples of $(\pi)^{-n / 2} \operatorname{Vol}\left(T^{n}\right)$. This agrees with the explicit formula in [BRMA06, (4.43)].

Remark 2.10. In general, the metric anomaly in [BRMA06] is expressed in terms of the curvature tensor, whereas our result provides an expression in terms of residues of the shifted zeta function $\zeta_{k, N}(s)$. The residues can be computed in terms of the coefficients of the asymptotic expansion of the trace of the heat operator of the Laplacian of $N$ on forms. It is well-known that these coefficients are given by the integral of universal polynomials in the covariant derivatives of the curvature tensor.

An immediate corollary is the identification of the Ray-Singer metric for a bounded generalized cone with exact cone metric near the singularity and product metric near the regular boundary.

Corollary 2.11. Let $(C(N), g)$ be a cone over a closed oriented Riemannian manifold $\left(N^{n}, g^{N}\right)$ of even dimension. Let $\left(E, \nabla, h^{E}\right)$ be a flat complex Hermitian vector bundle over $C(N)$ and let $\left(E_{N}, \nabla_{N}, h_{N}\right)$ be the associated flat vector bundle over $N$. Let $g_{0}$ be a metric on $C(N)$ which coincides with $d x^{2}+x^{2} g^{N}$ near the singularity at $x=0$ and with the product metric $d x^{2} \oplus g^{N}$ near the boundary $\{1\} \times N$. Then

$$
\log \left(\frac{\|\cdot\|_{\left(C(N), E, g_{0}\right)}^{R S}}{\|\cdot\|_{\operatorname{det} H^{*}(C(N), E)}}\right)=\operatorname{Top}\left(N, E_{N} ; g^{N}\right)+\operatorname{Tors}\left(N, E_{N} ; g^{N}\right),
$$

where $\operatorname{Top}\left(N, E_{N} ; g^{N}\right)$ and $\operatorname{Tors}\left(N, E_{N} ; g^{N}\right)$ are given by (2.13) and (2.14), respectively.

Corollary 2.11 shows that the main contribution of the conical singularity to the analytic torsion is given by $\operatorname{Tors}\left(N, E_{N} ; g^{N}\right)$. This result will be of significance in regard of the splitting formula.

Next we study its asymptotic behavior under scaling of the metric of $N$.

Proposition 2.12. Let $\left(N, g^{N}\right)$ be a closed oriented Riemannian manifold and let $g_{\mu}^{N}:=$ $\mu^{-2} g^{N}, \mu>0$. Then

$$
\operatorname{Tors}\left(N, E_{N} ; g_{\mu}^{N}\right)=O\left(\frac{\log \mu}{\mu}\right), \quad \mu \rightarrow \infty .
$$

\section{Variation of Analytic Torsion under Higher Order Deformations}

In this section we recall some facts about the asymptotic properties of the heat kernel on a manifold with conical singularities. Then we will show that the Ray-Singer metric is invariant under higher order deformations of the metric near the tip of the cone. 
Let $\left(M, g^{M}\right)$ be an odd-dimensional compact Riemannian manifold with an isolated conical singularity with $M=\mathscr{U} \cup_{N} X$ and metric on $\mathscr{U}$ given by (2.1). Let $\left(E, \nabla, h^{E}\right)$ be a flat Hermitian vector bundle over $M$ and let $\left(E_{N}, \nabla_{N}, h_{N}\right)$ be the flat Hermitian vector bundle over $N$ associated to $E$ via (2.3).

Let $\left(\Omega^{*}(\mathscr{U}, E), d_{*}\right)$ be the twisted de Rham complex over $\mathscr{U}$. In this section we assume essential self-adjointness of the Gauß-Bonnet operator, so that the Laplacian $\Delta_{k}$ defined with respect to relative or absolute boundary conditions coincides with the Frederich's extension $\Delta_{k}^{\mathscr{F}}$ in all degrees $k=0, \ldots, m$. Essential self-adjointness of the Gauß-Bonnet operator can always be achieved by scaling $g^{N}(0)$ to $c^{2} g^{N}(0)$ with $c>0$ sufficiently small, see [BRLE93].

Remark 3.1. The variational result of this section holds in fact for any self-adjoint extension of the Laplacian satisfying Theorem 3.4, which does hold for the Frederich's extension $\Delta^{\mathscr{F}}$. However it suffices to impose the Witt condition $H^{p}\left(N, E_{N}\right)=0$, $p=(m-1) / 2$ instead of essential self-adjointness of the Gauß-Bonnet operator for Theorem 3.4 to hold for the relative and absolute self-adjoint extension. We confine ourselves here to the case of essentially self-adjoint Gauß-Bonnet operator for simplicity.

We use the unitary rescaling transformation over $\mathscr{U}$, introduced in [BRSE88, (5.2)]

$$
\begin{array}{r}
\Psi_{k}: C_{c}^{\infty}\left((0,1), \Omega^{k-1}\left(N, E_{N}\right) \oplus \Omega^{k}\left(N, E_{N}\right)\right) \rightarrow \Omega_{c}^{k}(\mathscr{U}, E) \\
\left(\phi_{k-1}, \phi_{k}\right) \mapsto x^{k-1-n / 2} \phi_{k-1} \wedge d x+x^{k-n / 2} \phi_{k},
\end{array}
$$

where $\phi_{k}, \phi_{k-1}$ are identified with their pullback to $\mathscr{U}$ under the projection $\pi: \mathscr{U} \cong$ $(0,1) \times N \rightarrow N$ onto the second factor, and $x \in(0,1)$ is the radial coordinate.

Proposition 3.2. [BRSE88, (5.4)] The rescaling map $\Phi_{k}$ extends to a unitary transformation

$$
L^{2}\left((0,1), L^{2}\left(\Omega^{k-1}\left(N, E_{N}\right) \oplus \Omega^{k}\left(N, E_{N}\right), g^{N}(x), h_{N}\right)\right) \rightarrow L^{2}\left(\Omega^{k}(\mathscr{U}, E),\left.g^{M}\right|_{\mathscr{U}},\left.h^{E}\right|_{\mathscr{U}}\right) .
$$

Throughout this paper we will use the unitary transformation (3.1) and denote the heat operator under the unitary transformation again by $e^{-t \Delta_{k}}$. By a small abuse of notation we denote the heat operator and the corresponding heat kernel both by $e^{-t \Delta_{k}}$.

3.1. The Heat Kernel on Manifolds with Conical Singularities. In order to describe the asymptotic properties of the heat kernel $e^{-t \Delta_{k}}$ accurately, we consider $M$ as the interior of a compact manifold $\bar{M}$ with boundary.

The heat kernel of $e^{-t \Delta_{k}}$ is a priori a $k$-form valued distribution on a manifold with corners $M_{h}^{2}=\mathbb{R}^{+} \times \bar{M}^{2}$. Its singular structure can be conveniently described by lifting $e^{-t \Delta_{k}}$ to the heat space $\mathscr{M}_{h}^{2}$, which is a resolution of $M_{h}^{2}$ obtained by blowing up certain submanifolds of its boundary, see ([Moo99]). We begin by a blowup of

$$
A=\{t=0\} \times(\partial \bar{M})^{2} \subset M_{h}^{2},
$$

where the $\mathbb{R}^{+}$-direction is scaled parabolically, according to the parabolic homogeneity of the problem. The resulting space $\left[M_{h}^{2}, A\right]$ is defined as the union of $\left(M_{h}^{2} \backslash A\right)$ with the interior spherical normal bundle of $A$ in $M_{h}^{2}$. The blowup $\left[M_{h}^{2}, A\right]$ is endowed with the unique minimal differential structure, including $\sqrt{t}$, smooth functions in the interior of $M_{h}^{2}$ and polar coordinates around $A$. The blowup introduces an additional boundary 
hypersurface, which we refer to as the front face ff. We refer to [MEL93] for a careful definition and general discussion of parabolic blowups.

The actual heat-space $\mathscr{M}_{h}^{2}$ is obtained by blowing up $\left[M_{h}^{2}, A\right]$ at the lift of the diagonal in $\bar{M}^{2}$ at $t=0$, again parabolically in the time $\mathbb{R}^{+}$-direction. This blowup introduces a boundary hypersurface, which we refer to as the temporal diagonal td. The heat space comes with a canonical blowdown map $\beta: \mathscr{M}_{h}^{2} \rightarrow M_{h}^{2}$ and may be visualized as follows.

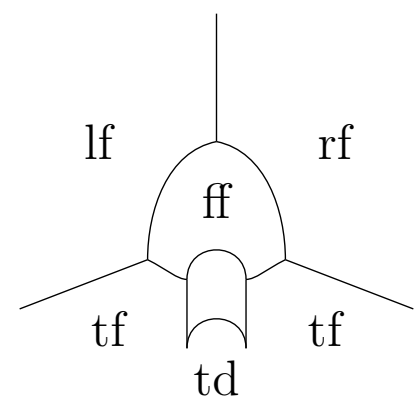

Figure 1. Heat-space Blowup $\mathscr{M}_{h}^{2}$.

We fix coordinate chart $(x, z)$ in the singular neighborhood $\mathscr{U} \subset M$. Two copies of this chart together with the time coordinate yield a coordinate system $(t, x, z, \widetilde{x}, \widetilde{z})$ on $M_{h}^{2}$. The projective coordinates on $\mathscr{M}_{h}^{2}$ are then given as follows. Near the top corner of the front face $\mathrm{ff}$, the projective coordinates are given by

$$
\rho=\sqrt{t}, \xi=x / \rho, \widetilde{\xi}=\widetilde{x} / \rho, z, \widetilde{z}
$$

where in these coordinates $\rho, \xi, \widetilde{\xi}$ are the defining functions of the boundary faces ff, lf and rf respectively. For the right hand side bottom corner of the front face projective coordinates are given by

$$
\tau=t / \widetilde{x}^{2}, s=x / \widetilde{x}, z, \widetilde{x}, \widetilde{z}
$$

where in these coordinates $\tau, s, \widetilde{x}$ are the defining functions of tf, rf and ff respectively. For the left hand side bottom corner of the front face projective coordinates are obtained by interchanging the roles of $x$ and $\widetilde{x}$. Projective coordinates on $\mathscr{M}_{h}^{2}$ near temporal diagonal are given by

$$
\eta=\frac{\sqrt{t}}{\widetilde{x}}, S=\frac{(x-\widetilde{x})}{\sqrt{t}}, Z=\frac{\widetilde{x}(z-\widetilde{z})}{\sqrt{t}}, \widetilde{x}, \widetilde{z} .
$$

In these coordinates tf is the face in the limit $|(S, Z)| \rightarrow \infty$, ff and td are defined by $\widetilde{x}, \eta$, respectively. The blow-down map $\beta: \mathscr{M}_{h}^{2} \rightarrow M_{h}^{2}$ is in local coordinates simply the coordinate change back to $(t,(x, z),(\widetilde{x}, \widetilde{z}))$. The heat kernel lifts to a polyhomogeneous conormal distribution on $\mathscr{M}_{h}^{2}$ with product type expansions at the corners of the heat space, and with coefficients depending smoothly on the tangential variables. More precisely we have the following definition.

Definition 3.3. Let $\mathfrak{W}$ be a manifold with corners, with all boundary faces embedded, and $\left\{\left(H_{i}, \rho_{i}\right)\right\}_{i=1}^{N}$ an enumeration of its boundaries and the corresponding defining 
functions. For any multi-index $b=\left(b_{1}, \ldots, b_{N}\right) \in \mathbb{C}^{N}$ we write $\rho^{b}=\rho_{1}^{b_{1}} \ldots \rho_{N}^{b_{N}}$. Denote by $\mathcal{V}_{b}(\mathfrak{W})$ the space of smooth vector fields on $\mathfrak{W}$ which lie tangent to all boundary faces. A distribution $\omega$ on $\mathfrak{W}$ is said to be conormal, if $w \in \rho^{b} L^{\infty}(\mathfrak{W})$ for some $b \in \mathbb{C}^{N}$ and $V_{1} \ldots V_{\ell} u \in \rho^{b} L^{\infty}(\mathfrak{W})$ for all $V_{j} \in \mathcal{V}_{b}(\mathfrak{W})$ and for every $\ell \geq 0$. An index set $E_{i}=\{(\gamma, p)\} \subset \mathbb{C} \times \mathbb{N}$ satisfies the following hypotheses:

(i) $\operatorname{Re}(\gamma)$ accumulates only at plus infinity,

(ii) For each $\gamma$ there is $P_{\gamma} \in \mathbb{N}_{0}$, such that $(\gamma, p) \in E_{i}$ for $p \leq P_{\gamma}<\infty$,

(iii) If $(\gamma, p) \in E_{i}$, then $\left(\gamma+j, p^{\prime}\right) \in E_{i}$ for all $j \in \mathbb{N}$ and $0 \leq p^{\prime} \leq p$.

An index family $E=\left(E_{1}, \ldots, E_{N}\right)$ is an $N$-tuple of index sets. Finally, we say that a conormal distribution $w$ is polyhomogeneous on $\mathfrak{W}$ with index family $E$ (denoted by $\left.\omega \in \mathscr{A}_{\mathrm{phg}}^{E}(\mathfrak{W})\right)$, if $w$ is conormal and if in addition, near each $H_{i}$,

$$
w \sim \sum_{(\gamma, p) \in E_{i}} a_{\gamma, p} \rho_{i}^{\gamma}\left(\log \rho_{i}\right)^{p}, \text { as } \rho_{i} \rightarrow 0,
$$

with coefficients $a_{\gamma, p}$ conormal on $H_{i}$, polyhomogeneous with index $E_{j}$ at any $H_{i} \cap H_{j}$.

The asymptotic properties of the lift $\beta^{*} e^{-t \Delta_{k}}$ of the heat kernel to $\mathscr{M}_{h}^{2}$ in an open neighborhood of the front face have been described by Mooers in [Moo99].

Theorem 3.4. ([Moo99]) The lift $\beta^{*} e^{-t \Delta_{k}}$ is polyhomogeneous conormal distribution on $\mathscr{M}_{h}^{2}$ of leading order $(-1)$ at the front face ff, leading order $(-\operatorname{dim} M)$ at the temporal diagonal $t d$, and of leading order $1 / 2$ at the left and right boundary faces. $\beta^{*} e^{-t \Delta_{k}}$ vanishes to infinite order at the temporal face tf and does not admit logarithmic terms in its asymptotic expansion at ff and $t d$.

The asymptotic properties of the heat kernel $e^{-t \Delta_{k}}$ has been discussed by various authors, with major contributions by Brüning-Seeley in [BRSE85], Lesch [LES97] and Mooers [Moo99]. The heat calculus on manifolds with edges by Mazzeo-Vertman [MAVE10] contains the setup of isolated conical singularities as a special case.

3.2. Analytic Torsion under Metric Variation at the Cone Tip. We now study analytic torsion of $\left(M, g^{M}\right)$ under higher order deformations of the Riemannian metric $g^{M}$. Let $\mathscr{I} \subset \mathbb{R}$ be an open interval and let $\left(g_{\mu}^{M}, \mu \in \mathscr{I}\right)$ be a smooth family of Riemannian metrics on $M$ with

$$
g_{\mu}^{M} \uparrow \mathscr{U}=d x^{2} \oplus x^{2} g^{N}(x, \mu), g^{N} \in C^{\infty}\left([0,1] \times \mathscr{I}, \operatorname{Sym}^{2}\left(T^{*} N\right)\right) .
$$

The dependence of $\Delta_{k}$ on the parameter is denoted by $\Delta_{k}(\mu)$. For $\mu \neq \mu_{0}$ the corresponding operators $\Delta_{k}(\mu), \Delta_{k}\left(\mu_{0}\right)$ act on different Hilbert spaces $L^{2} \Omega^{k}\left(M, g_{\mu}^{M}\right)$ and $L^{2} \Omega^{k}\left(M, g_{\mu_{0}}^{M}\right)$, respectively. We fix some $\mu_{0} \in \mathscr{I}$ and employ the natural isometry

$$
T_{\mu}: L^{2} \Omega^{k}\left(M, g_{\mu}^{M}\right) \rightarrow L^{2} \Omega^{k}\left(M, g_{\mu_{0}}^{M}\right)
$$

to define self-adjoint operators in the fixed Hilbert space $L^{2} \Omega^{k}\left(M, g_{\mu_{0}}^{M}\right)$

$$
H_{k}(\mu):=T_{\mu} \circ \Delta_{k}(\mu) \circ T_{\mu}^{-1} .
$$


As a simple consequence of the semi-group property we have for any $\mu, \mu_{0} \in \mathscr{I}$

$$
\begin{aligned}
\frac{e^{-t H_{k}(\mu)}-e^{-t H_{k}\left(\mu_{0}\right)}}{\mu-\mu_{0}} & =\int_{0}^{t} \frac{\partial}{\partial s}\left(\frac{e^{-(t-s) H_{k}\left(\mu_{0}\right)} e^{-s H_{k}(\mu)}}{\mu-\mu_{0}}\right) d s \\
& =\int_{0}^{t} e^{-(t-s) H_{k}\left(\mu_{0}\right)}\left(\frac{H_{k}\left(\mu_{0}\right)-H_{k}(\mu)}{\mu-\mu_{0}}\right) e^{-s H_{k}(\mu)} d s .
\end{aligned}
$$

Taking the limit $\mu \rightarrow \mu_{0}$ this leads to

$$
\begin{aligned}
\left.\frac{\partial}{\partial \mu} \operatorname{Tr}\left(e^{-t H_{k}(\mu)}\right)\right|_{\mu=\mu_{0}} & =-\int_{0}^{t} \operatorname{Tr}\left(e^{-(t-s) H_{k}\left(\mu_{0}\right)}\left(\dot{H}_{k}\left(\mu_{0}\right)\right) e^{-s H_{k}\left(\mu_{0}\right)}\right) \\
& =-t \cdot \operatorname{Tr}\left(\left(\dot{H}_{k}\left(\mu_{0}\right)\right) e^{-t H_{k}\left(\mu_{0}\right)}\right)
\end{aligned}
$$

where the upper-dot denotes the derivative with respect to $\mu$. Evaluating $\dot{H}_{k}(\mu)$ explicitly in terms of the isometry $T_{\mu}$, we find

$$
\begin{aligned}
\left.\frac{\partial}{\partial \mu} \operatorname{Tr}\left(e^{-t H_{k}(\mu)}\right)\right|_{\mu=\mu_{0}}= & -t \cdot \operatorname{Tr}\left(\dot{T}_{\mu_{0}} \circ \Delta_{k}\left(\mu_{0}\right) \circ e^{-t \Delta_{k}\left(\mu_{0}\right)} \circ T_{\mu_{0}}^{-1}\right) \\
& -t \cdot \operatorname{Tr}\left(\Delta_{k}\left(\mu_{0}\right) \circ \dot{T}_{\mu_{0}}^{-1} T_{\mu_{0}} \circ e^{-t \Delta_{k}\left(\mu_{0}\right)}\right) \\
& -t \cdot \operatorname{Tr}\left(\dot{\Delta}_{k}\left(\mu_{0}\right) \circ e^{-t \Delta_{k}\left(\mu_{0}\right)}\right) .
\end{aligned}
$$

For the second summand in (3.7) we employ commutativity of bounded operators under the trace and find

$$
\begin{aligned}
& \operatorname{Tr}\left(\Delta_{k}\left(\mu_{0}\right) \circ \dot{T}_{\mu_{0}}^{-1} T_{\mu_{0}} \circ e^{-t \Delta_{k}\left(\mu_{0}\right)}\right)=\operatorname{Tr}\left(e^{-t / 2 \Delta_{k}\left(\mu_{0}\right)} \Delta_{k}\left(\mu_{0}\right) \circ \dot{T}_{\mu_{0}}^{-1} T_{\mu_{0}} \circ e^{-t / 2 \Delta_{k}\left(\mu_{0}\right)}\right) \\
= & \operatorname{Tr}\left(\dot{T}_{\mu_{0}}^{-1} T_{\mu_{0}} \circ \Delta_{k}\left(\mu_{0}\right) \circ e^{-t \Delta_{k}\left(\mu_{0}\right)}\right)=-\operatorname{Tr}\left(T_{\mu_{0}}^{-1} \dot{T}_{\mu_{0}} \circ \Delta_{k}\left(\mu_{0}\right) \circ e^{-t \Delta_{k}\left(\mu_{0}\right)}\right) .
\end{aligned}
$$

Consequently the first and the second summands in (3.7) cancel and we get

$$
\left.\frac{\partial}{\partial \mu} \operatorname{Tr}\left(e^{-t \Delta_{k}(\mu)}\right)\right|_{\mu=\mu_{0}}=-t \cdot \operatorname{Tr}\left(\dot{\Delta}_{k}\left(\mu_{0}\right) \circ e^{-t \Delta_{k}\left(\mu_{0}\right)}\right) .
$$

Let $P_{k}(\mu), \mu \in \mathscr{I}$ denote the orthogonal projection onto the kernel of $\Delta_{k}(\mu)$. Let $*_{\mu}$ denote the Hodge-star operator associated to $g_{\mu}^{M}, \mu \in \mathscr{I}$, and put $\alpha_{\mu}^{k}:=*_{\mu}^{-1} \dot{*}_{\mu}$, with the upper-index $k$ denoting the restriction to forms of degree $k$. Repeating the arguments of Ray-Singer in [RASi71, p. 152-153] we get

$$
\begin{aligned}
& \frac{\partial}{\partial \mu} \sum_{k=0}^{\operatorname{dim} M}(-1)^{k} \cdot k \cdot \operatorname{Tr}\left(e^{-t \Delta_{k}(\mu)}-P_{k}(\mu)\right) \\
= & t \frac{\partial}{\partial t} \sum_{k=0}^{\operatorname{dim} M}(-1)^{k} \operatorname{Tr}\left(\alpha_{\mu}^{k}\left(e^{-t \Delta_{k}(\mu)}-P_{k}(\mu)\right)\right) d t .
\end{aligned}
$$

Put

$$
f(\mu, s):=\frac{1}{2} \sum_{k=0}^{\operatorname{dim} M}(-1)^{k} \cdot k \cdot \frac{1}{\Gamma(s)} \int_{0}^{\infty} t^{s-1} \operatorname{Tr}\left(e^{-t \Delta_{k}(\mu)}-P_{k}(\mu)\right) d t .
$$


Then by definition we have

$$
\log T\left(M, E, g_{\mu}^{M}\right)=\left.\frac{\partial}{\partial s}\right|_{s=0} f(\mu, s) .
$$

Since the heat trace is exponentially decaying, we can differentiate $f(\mu, s)$ with respect to $\mu \in I$ in the half-plane $\operatorname{Re}(s) \gg 0$, by differentiating under the integral sign. Choosing $\operatorname{Re}(s)$ large enough so that boundary terms vanish and using (3.9) we get

$$
\begin{aligned}
\frac{\partial}{\partial \mu} f(\mu, s) & =\frac{1}{2} \sum_{k=0}^{\operatorname{dim} M}(-1)^{k} \frac{1}{\Gamma(s)} \int_{0}^{\infty} t^{s} \frac{d}{d t} \operatorname{Tr}\left(\alpha_{\mu}^{k}\left(e^{-t \Delta_{k}(\mu)}-P_{k}(\mu)\right)\right) d t \\
& =\frac{1}{2} s \sum_{k=0}^{\operatorname{dim} M}(-1)^{k+1} \frac{1}{\Gamma(s)} \int_{0}^{\infty} t^{s-1} \operatorname{Tr}\left(\alpha_{\mu}^{k}\left(e^{-t \Delta_{k}(\mu)}-P_{k}(\mu)\right)\right) d t .
\end{aligned}
$$

Let us henceforth assume that the metric family $g_{\mu}^{M}$ differs only in its higher order terms on the singular neighborhood $\mathscr{U}$. More precisely, assume that for all $\mu \in \mathscr{I}$

$$
g^{N}(0, \mu) \equiv g^{N}(0)
$$

Under the transformation (3.1), $\left.\alpha_{\mu}^{k}\right|_{\mathscr{U}} \in C^{\infty}\left([0,1]\right.$, End $\left.\left(\Omega^{k-1}\left(N, E_{N}\right) \oplus \Omega^{k}\left(N, E_{N}\right)\right)\right)$ for any $\mu \in \mathscr{I}$, and by (3.12), it follows that

$$
\alpha_{\mu}^{k}(x)=O(x), \quad x \rightarrow 0 .
$$

Let $\pi: \mathbb{R}^{+} \times \bar{M}^{2} \rightarrow \bar{M}$ be the projection onto a copy of $\bar{M}$. Then, employing the projective coordinates on the heat space $\mathscr{M}_{h}^{2}$, the lift $(\pi \circ \beta)^{*} \alpha_{\mu}^{k}$ is a polyhomogeneous function on $\mathscr{M}_{h}^{2}$ with

$$
(\pi \circ \beta)^{*} \alpha_{\mu}^{k}=\rho_{\mathrm{ff}} \rho_{\mathrm{rf}} \cdot \mathscr{B}
$$

where $\rho_{\text {ff }}$ and $\rho_{\text {rf }}$ are boundary defining functions of the front and the right boundary face, respectively; $\mathscr{B}$ is a polyhomogeneous bounded function on $\mathscr{M}_{h}^{2}$. If we compose $(\pi \circ \beta)^{*} \alpha_{\mu}^{k}$ pointwise with $\beta^{*} e^{-t \Delta_{k}(\mu)}$ and use Theorem 3.4, we obtain the following lemma.

Lemma 3.5. The lift $\beta^{*}\left(\alpha_{\mu}^{k} e^{-t \Delta_{k}(\mu)}\right)$ is a polyhomogeneous conormal distribution on $\mathscr{M}_{h}^{2}$ of zero leading order at the front face ff, leading order $(-\operatorname{dim} M)$ at the temporal diagonal $t d$, and of leading order $1 / 2$ and $3 / 2$ at the left and right boundary faces, respectively. $\beta^{*}\left(\alpha_{\mu}^{k} e^{-t \Delta_{k}(\mu)}\right)$ vanishes to infinite order at the temporal face $t f$ and does not admit logarithmic terms in its asymptotic expansion at ff and $t$.

For any fixed $t>0$

$$
\alpha_{\mu}^{k} e^{-t \Delta_{k}(\mu)} \in \wedge^{k} T^{*} M \otimes \wedge^{k} T^{*} M \cong\left(\wedge^{k} T^{*} M\right)^{*} \otimes \wedge^{k} T^{*} M
$$

The right hand side of (3.14) admits on the diagonal an invariantly defined pointwise trace tr, and $\operatorname{tr}\left(\alpha_{\mu}^{k} e^{-t \Delta_{k}(\mu)}\right)$ defines a polyhomogeneous conormal distribution on the lift $\mathscr{D}$ of the diagonal in $\bar{M}^{2}$ to the heat space $\mathscr{M}_{h}^{2}$. The lifted diagonal $\mathscr{D}$ is a resolution of $\mathbb{R}^{+} \times \bar{M}$, obtained by blowing up $\{0\} \times \partial \bar{M}$, with the $\mathbb{R}^{+}$-direction scaled parabolically; $\mathscr{D}$ may be visualized as in Figure 3.2. 


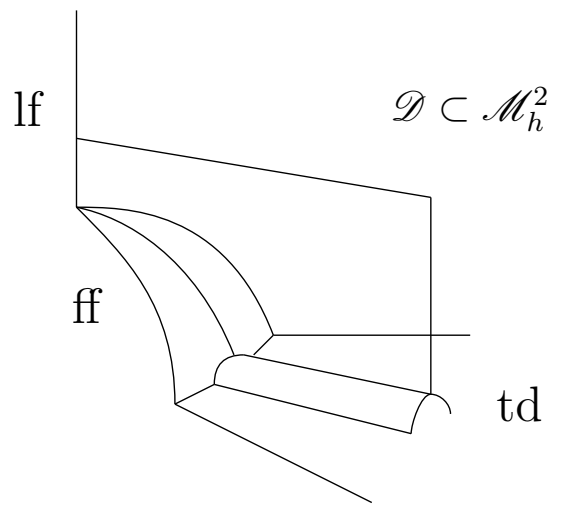

Figure 2. The lifted diagonal $\mathscr{D}$ of the heat-space $\mathscr{M}_{h}^{2}$.

By a small abuse of notation, we refer to the boundary faces of the lifted diagonal $\mathscr{D} \subset \mathscr{M}_{h}^{2}$ again as temporal diagonal td, front face ff and left face lf, respectively. The projective coordinates (3.3) and (3.5) on $\mathscr{M}_{h}^{2}$ yield projective coordinates on $\mathscr{D}$. Near the top corner of $\mathscr{D}$ the projective coordinates are given by

$$
\rho=\sqrt{t}, \xi=x / \rho, z,
$$

where in these coordinates $\rho$ and $\xi$ are the defining functions of the front and the left boundary face, respectively. Near the lower corner of $\mathscr{D}$ the projective coordinates are given by

$$
\eta=\sqrt{t} / x, x, z,
$$

where in these coordinates $x$ and $\eta$ are the defining functions of the front and the temporal diagonal boundary face, respectively. $\mathscr{D}$ comes with a canonical blowdown map $\beta_{\mathscr{D}}: \mathscr{D} \rightarrow \mathbb{R}^{+} \times \bar{M}$, which in local coordinates is simply the coordinate change back to $(t, x, z)$.

The identification of the exterior bundle $\wedge^{k} T^{*} M$ with its dual $\left(\wedge^{k} T^{*} M\right)^{*}$ via $g^{M}$ in fact does not induce any shift in the polyhomogeneous expansion of $\alpha_{\mu}^{k} e^{-t \Delta_{k}(\mu)}$, since we consider the setup after unitary transformation in Proposition 3.2 to a product $L^{2}$-space. By Lemma 3.5 we arrive at the following

Proposition 3.6. The lift $\beta_{\mathscr{D}}^{*} \operatorname{tr}\left(\alpha_{\mu}^{k} e^{-t \Delta_{k}(\mu)}\right)$ is a polyhomogeneous conormal distribution on $\mathscr{D}$ of zero leading order at the front face ff, leading order $(-\operatorname{dim} M)$ at the temporal diagonal $t d$, and of leading order 2 at the left boundary face lf. Moreover the asymptotic expansion at ff does not admit logarithmic terms and the expansion at $t d$ ( $\rho_{\mathrm{td}}$ denotes the defining function of $t d$ ) is of the form

$$
\beta_{\mathscr{D}}^{*} \operatorname{tr}\left(\alpha_{\mu}^{k} e^{-t \Delta_{k}(\mu)}\right) \sim \rho_{\mathrm{td}}^{-\operatorname{dim} M}\left(\sum_{k=0}^{\infty} a_{2 k} \rho_{\mathrm{td}}^{2 k}\right), \text { as } \rho_{\mathrm{td}} \rightarrow 0 .
$$

Proof. The polyhomogeneity and the leading orders in the asymptotic expansions of $\beta_{\mathscr{D}}^{*} \operatorname{tr}\left(\alpha_{\mu}^{k} e^{-t \Delta_{k}(\mu)}\right)$ at the boundary faces of $\mathscr{D}$ follow by Lemma 3.5. Moreover, at the 
temporal diagonal Lemma 3.5 implies

$$
\beta_{\mathscr{D}}^{*} \operatorname{tr}\left(\alpha_{\mu}^{k} e^{-t \Delta_{k}(\mu)}\right) \sim \rho_{\mathrm{td}}^{-\operatorname{dim} M}\left(\sum_{k=0}^{\infty} a_{k} \rho_{\mathrm{td}}^{k}\right), \text { as } \rho_{\mathrm{td}} \rightarrow 0 .
$$

We have the following classical pointwise trace expansion on Riemannian manifolds

$$
\operatorname{tr}\left(\alpha_{\mu}^{k} e^{-t \Delta_{k}(\mu)}\right) \sim(\sqrt{t})^{-\operatorname{dim} M} \sum_{k=0}^{\infty} \alpha_{2 k}(\sqrt{t})^{2 k}, \text { as } t \rightarrow 0 .
$$

Employing the projective coordinates (3.15) and (3.16) we find $\beta_{\mathscr{D}}^{*} t=\rho_{\mathrm{ff}}^{2} \rho_{\mathrm{td}}^{2}$. Hence, lifting (3.19) to $\mathscr{D}$, we deduce for (3.18) that $a_{k} \equiv 0$ for $k$ odd.

Suppose $\mu$ is a density on $\mathscr{D}$, which is smooth up to all boundary faces and nowhere vanishing. A smooth $b$-density $\mu_{b}$ is by definition any density of the form $\left(\rho_{\mathrm{ff}} \rho_{\mathrm{lf}} \rho_{\mathrm{td}}\right)^{-1} \mu$. Let $\pi_{\mathscr{D}}: \mathbb{R}^{+} \times \bar{M} \rightarrow \mathbb{R}^{+}$is the projection onto the first factor. We can push forward densities in a natural way and a straightforward computation in local coordinates shows

$$
\left(\pi_{\mathscr{D}} \circ \beta_{\mathscr{D}}\right)_{*} \beta_{\mathscr{D}}^{*}\left(x \cdot \operatorname{tr}\left(\alpha_{\mu}^{k} e^{-t \Delta_{k}(\mu)}\right)\right)=\operatorname{Tr}\left(\alpha_{\mu}^{k} e^{-t \Delta_{k}(\mu)}\right) f(t) \frac{d t}{t},
$$

where $f$ is some smooth function on $\mathbb{R}^{+} \cup\{0\}$. We derive the short-time asymptotics of $\operatorname{Tr}\left(\alpha_{\mu}^{k} e^{-t \Delta_{k}(\mu)}\right)$ employing the Push-forward theorem of Melrose [MEL92]. We also refer to [MEL92] for the exact definition of b-fibrations and b-densities.

Theorem 3.7. (Melrose, [MeL92]) Let $\mathfrak{W}_{1}, \mathfrak{W}_{2}$ be manifolds with corners, and let $\left\{\left(H_{i}, \rho_{i}\right)\right\}_{i \in I}$ and $\left\{\left(K_{j}, \delta_{j}\right)\right\}_{j \in J}$ be the respective indexing of the boundaries and the corresponding defining functions. Let $f: \mathfrak{W}_{1} \rightarrow \mathfrak{W}_{2}$ be a b-fibration with

$$
f^{*} \delta_{j}=h \cdot \prod_{i \in I} \rho_{j}^{e(j, i)},
$$

where $h \in C^{\infty}\left(\mathfrak{W}_{1}\right)$ is strictly positive and the exponents $e(j, i) \in \mathbb{N}_{0}$ are such that for any $j \in J$ there is at most one $i \in I$ with $e(j, i) \neq 0$. If $\omega \in \mathscr{A}_{\mathrm{phg}}^{E}\left(\mathfrak{W}_{1}\right)$ for an index set $E=\left(E_{i}\right)_{i \in I}$ such that $f_{*} \omega$ exists, then $f_{*} \omega \in \mathscr{A}_{\mathrm{phg}}^{f_{*} E}\left(\mathfrak{W}_{2}\right)$, with the index family $f_{*} E=\left(f_{*} E\right)_{j \in J}$, where the index sets $\left(f_{*} E\right)_{j}=\{(\gamma, p)\} \in \mathbb{C} \times \mathbb{N}_{0}$ are comprised of all pairs $(\gamma, p)$ such that

(i) $(\gamma, p)=(s / e(j, i), l)$, for $(s, l) \in E_{i}$ with $e(j, i) \neq 0$,

(ii) $(\gamma, p)=\left(s / e(j, i), l+l^{\prime}+1\right)$, for $(s, l) \in E_{i}$ and $\left(s^{\prime}, l^{\prime}\right) \in E_{i^{\prime}}$ with $e(j, i), e\left(j, i^{\prime}\right) \neq 0$ and $s / e(j, i)=s^{\prime} / e\left(j, i^{\prime}\right)$.

In the projective coordinates (3.15) and (3.16) we directly verify that the b-fibration $\left(\pi_{\mathscr{D}} \circ \beta_{\mathscr{D}}\right)$ satisfies $\left(\pi_{\mathscr{D}} \circ \beta_{\mathscr{D}}\right)^{*} t=\rho_{\mathrm{ff}}^{2} \rho_{\mathrm{td}}^{2}$. Hence, by Push-forward Theorem 3.7 we infer from (3.20) and Proposition 3.6

$$
\operatorname{Tr}\left(\alpha_{\mu}^{k} e^{-t \Delta_{k}(\mu)}\right) \sim \sum_{j=0}^{\infty} A_{j} t^{j-\frac{m}{2}}+\sum_{j=1}^{\infty} C_{j} t^{\frac{j}{2}}+\sum_{j=1}^{\infty} G_{j} t^{\frac{j}{2}} \log t, \text { as } t \rightarrow 0 .
$$

Consequently

$$
\operatorname{Res}_{s=0} \int_{0}^{\infty} t^{s-1} \operatorname{Tr}\left(\alpha_{\mu}^{k}\left(e^{-t \Delta_{k}(\mu)}-P_{k}(\mu)\right)\right) d t=-\operatorname{Tr}\left(\alpha_{\mu}^{k} P_{k}(\mu)\right) .
$$


In view of the additional $s$-factor in (3.11), we find by (3.10)

$$
\begin{aligned}
\frac{d}{d \mu} \log T\left(M, E, g_{\mu}^{M}\right) & =\frac{1}{2} \sum_{k=0}^{\operatorname{dim} M}(-1)^{k} \operatorname{Tr}\left(\alpha_{\mu}^{k} P_{k}(\mu)\right) \\
& =\frac{d}{d \mu} \log \|\cdot\|_{\operatorname{det} H^{*}(M, E), g_{\mu}^{M}}^{-1}
\end{aligned}
$$

We have proved the following main result (Theorem 2.5) of this section.

Theorem 3.8. Let $\left(M, g_{\mu}^{M}\right), \mu \in \mathscr{I}$ be a compact odd-dimensional Riemannian manifold with an isolated conical singularity and a singular neighborhood $\mathscr{U} \cong(0,1) \times N$, endowed with a family of conical metrics, parametrized over an open $\mathscr{I} \subset \mathbb{R}$, such that

$$
g_{\mu}^{M} \uparrow \mathscr{U}=d x^{2} \oplus x^{2} g^{N}(x, \mu), g^{N} \in C^{\infty}\left([0,1] \times \mathscr{I}, \operatorname{Sym}^{2}\left(T^{*} N\right)\right) .
$$

Let $\left(E, \nabla, h^{E}\right)$ be a flat Hermitian vector bundle over $M$ and let $\left(E_{N}, \nabla_{N}, h_{N}\right)$ be the

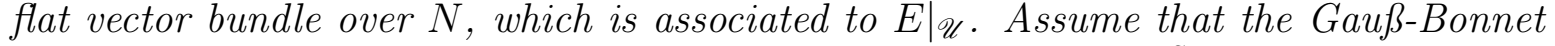
operator is essential self-adjoint. Then the Ray-Singer norm $\|\cdot\|_{\left(M, E ; g_{\mu}^{M}\right)}^{R S}$ is independent of $\mu$, i.e.

$$
\frac{d}{d \mu}\|\cdot\|_{\left(M, E ; g_{\mu}^{M}\right)}^{R S}=0
$$

\section{Scaling Invariance of the Metric Anomaly for Analytic Torsion}

To begin with we need to introduce some notation. Let $\mathscr{A}, \mathscr{B}$ be two $\mathbb{Z}_{2}$-graded algebras with identity and let $\mathscr{A} \widehat{\otimes} \mathscr{B}$ denote their $\mathbb{Z}_{2}$-graded tensor product. Identify $\mathscr{A}$ with $\mathscr{A} \widehat{\otimes} I$ and let $\widehat{\mathscr{B}}:=I \widehat{\otimes} \mathscr{B}$. Write $\wedge:=\widehat{\otimes}$ so that $\mathscr{A} \widehat{\otimes} \mathscr{B}=\mathscr{A} \wedge \mathscr{B}$.

Let $\left(X, g^{X}\right)$ be a compact oriented Riemannian manifold of odd dimension $m$ with boundary $\partial X$. In [BrMA06, (1.15)] elements

$$
\dot{R}^{T \partial X} \in \Lambda T^{*} \partial X \widehat{\otimes} \widehat{\Lambda T^{*} \partial X} \quad \text { and } \quad \dot{S} \in \Lambda T^{*} \partial X \widehat{\otimes} \widehat{\Lambda T^{*} \partial X}
$$

have been introduced. They are defined as follows. Let $R^{T \partial X}$ be the curvature tensor of $\left(\partial X, g^{\partial X}\right)$ and denote by $\left\{e_{k}\right\}_{k=1}^{m}$ a local orthonormal frame field on $\left(T X, g^{\partial X}\right)$. We assume that near the boundary $e_{m}$ is the inward-pointing unit normal vector at every boundary point. Let $\left\{e_{k}^{*}\right\}_{k=1}^{m}$ be the dual orthonormal frame field of $T^{*} X$ and let $\widehat{e_{k}^{*}}$ be the canonical identification with an element of $\widehat{\Lambda T^{*} X}$. Let $j: Y \hookrightarrow X$ be the canonical embedding. Then

$$
\dot{R}^{T \partial X}:=\frac{1}{2} \sum_{1 \leq k, j \leq m-1}\left\langle e_{k}, R^{T \partial X} e_{j}\right\rangle \widehat{e_{k}^{*}} \wedge \widehat{e_{j}^{*}} \quad \text { and } \quad \dot{S}:=\frac{1}{2} j^{*} \nabla^{T X} \widehat{e_{m}^{*}}
$$

$\dot{R}^{T \partial X}$ is defined in terms of the curvature of the Levi-Civita connection, induced by $g^{\partial X}$. $\dot{S}$ measures the deviation from a metric product structure near the boundary. $\dot{R}^{T \partial X}$ and $\dot{S}^{2}$ are both homogeneous of degree two. Let

$$
\int^{B_{\partial X}}: \Lambda T^{*} \partial X \widehat{\otimes} \widehat{\Lambda T^{*} \partial X} \rightarrow \Lambda T^{*} \partial X
$$


be the Berezin integral (see ([BRMA06, Section 1.1]). It is non-trivial only on elements which are homogeneous of degree $m$. Then the secondary class $B\left(\nabla^{T X}\right)$, introduced in $[$ BRMA06, (1.17)] is defined as follows

$$
B\left(\nabla^{T X}\right)=\int^{B \partial X} \exp \left(-\frac{1}{2} \dot{R}^{T \partial X}\right) \sum_{k=1}^{\infty} \frac{\left(-\dot{S}^{2}\right)^{k}}{4 k \Gamma(k+1)} .
$$

By([BRMA06, Theorem 0.1] the metric anomaly of analytic torsion is expressed in terms of $B\left(\nabla^{T X}\right)$. For our purpose we will need that $B\left(\nabla^{T X}\right)$ is scaling invariant. This is verified in the next proposition.

Proposition 4.1. Let $g_{1}^{X}$ be a Riemannian metrics on a compact manifold $(X, \partial X)$ with boundary. Let $s>0$ and put $g_{2}^{X}=s \cdot g_{1}^{X}$. Let $\nabla_{1}^{T X}, \nabla_{2}^{T X}$ denote the corresponding Levi-Civita connections. Then

$$
B\left(\nabla_{1}^{T X}\right)=B\left(\nabla_{2}^{T X}\right)
$$

Proof. Denote by $\left(\dot{R}_{i}^{T \partial X}\right.$ and $\dot{S}_{i}$ the elements (4.2) with respect to the metric $g_{i}^{T X}$, $i=1,2$. It follows from (4.2) that

$$
\dot{R}^{\partial T}\left(g_{2}^{X}\right)=s \dot{R}^{\partial T}\left(g_{1}^{X}\right), \quad \dot{S}\left(g_{2}^{X}\right)=\sqrt{s} \dot{S}\left(g_{1}^{X}\right) .
$$

The Berezin integral also depends on the metric. We denote it by $\int_{j}^{B_{\partial x}}, j=1,2$. Using the definition of the Berezin integral [BRMA06, (1.1)] we obtain

$$
\int_{2}^{B_{\partial X}}=s^{-\operatorname{dim} \partial X / 2} \int_{1}^{B_{\partial X}}
$$

Since $\dot{R}^{T \partial X}$ and $\dot{S}^{2}$ are both of degree two and the Berezin integral is non-trivial only on terms homogeneous of degree $\operatorname{dim} \partial X$, it follows from (4.6) that

$$
\begin{aligned}
B\left(\nabla_{2}^{T X}\right) & =\int_{2}^{B_{\partial X}} \exp \left(-\frac{1}{2} \dot{R}_{2}^{T \partial X}\right) \sum_{k=1}^{\infty} \frac{\left(-\dot{S}_{2}^{2}\right)^{k}}{4 k \Gamma(k+1)} \\
& =\int_{1}^{B_{\partial X}} \exp \left(-\frac{1}{2} \dot{R}_{2}^{T \partial X}\right) \sum_{k=1}^{\infty} \frac{\left(-\dot{S}_{2}^{2}\right)^{k}}{4 k \Gamma(k+1)} \cdot s^{-\operatorname{dim} \partial X / 2} \\
& =\int_{1}^{B_{\partial X}} \exp \left(-\frac{1}{2} \dot{R}_{1}^{T \partial X}\right) \sum_{k=1}^{\infty} \frac{\left(-\dot{S}_{1}^{2}\right)^{k}}{4 k \Gamma(k+1)}=B\left(\nabla_{1}^{T X}\right) .
\end{aligned}
$$

In the special case of a cone with a flat cross-section $N$ the scaling invariance of the metric anomaly follows also from [BRMA06, (4.43)].

\section{Analytic Torsion of the Truncated Cone}

5.1. Decomposition of the de Rham Complex of the Truncated Cone. The Analytic torsion of an exact cone can be evaluated by decomposing the de Rham complex into short subcomplexes. This decomposition has been used in the computation of analytic torsion of cones in [VER09A]. The same decomposition and its symmetry 
are in fact also valid for truncated cones. Let $I$ be either the open interval $(0,1)$ or the closed interval $[\varepsilon, 1], \varepsilon>0$. Let $\left(N^{n}, g^{N}\right)$ be an even-dimensional closed Riemannian manifold and let $C_{I}(N):=I \times N$, equipped with the metric

$$
g=d x^{2} \oplus x^{2} g^{N}, x \in I .
$$

Let $\left(E, \nabla, h^{E}\right)$ be a flat complex Hermitian vector bundle over $C_{I}(N)$ and $\left(E_{N}, \nabla_{N}, h_{N}\right)$ its restriction to the cross-section $N$. Let $\left(\Omega_{c}^{*}\left(C_{I}(N), E\right), d_{*}\right)$ be the associated twisted de Rham complex, where $\Omega_{c}^{*}\left(C_{I}(N), E\right)$ are the $E$-valued differential forms on $C_{I}(N)$ with compact support and $d_{*}$ denotes the differential. As in Section 3 we consider the map given by separation of variables

$$
\begin{array}{r}
\Psi_{k}: C_{c}^{\infty}\left(I, \Omega^{k-1}\left(N, E_{N}\right) \oplus \Omega^{k}\left(N, E_{N}\right)\right) \rightarrow \Omega_{c}^{k}\left(C_{I}(N), E\right) \\
\left(\omega_{k-1}, \omega_{k}\right) \mapsto x^{k-1-n / 2} \omega_{k-1} \wedge d x+x^{k-n / 2} \omega_{k},
\end{array}
$$

where $\omega_{k}, \omega_{k-1}$ are identified with their pullback to $C_{I}(N)$ under the projection $\pi$ : $I \times N \rightarrow N$ onto the second factor, and $x \in I \subset \mathbb{R}^{+}$. As in Proposition 3.2, the map $\Psi_{k}$ extends to an isometry

$$
\Psi_{k}: L^{2}\left(I, L^{2}\left(\Omega^{k-1}\left(N, E_{N}\right) \oplus \Omega^{k}\left(N, E_{N}\right), g^{N}, h_{N}\right), d x\right) \rightarrow L^{2}\left(\Omega_{c}^{k}\left(C_{I}(N), E\right), g, h^{E}\right) .
$$

Let

As in $[\mathrm{BrSE} 88,(5.5)]$ we obtain

$$
c_{k}:=(-1)^{k}\left(k-\frac{n}{2}\right), \quad k=0, \ldots, m .
$$

$$
\begin{array}{r}
\Psi_{k+1}^{-1} d_{k} \Psi_{k}=\left(\begin{array}{cc}
0 & (-1)^{k} \partial_{x} \\
0 & 0
\end{array}\right)+\frac{1}{x}\left(\begin{array}{cc}
d_{k-1, N} & c_{k} \\
0 & d_{k, N}
\end{array}\right), \\
\Psi_{k}^{-1} d_{k}^{*} \Psi_{k+1}=\left(\begin{array}{cc}
0 & 0 \\
(-1)^{k+1} \partial_{x} & 0
\end{array}\right)+\frac{1}{x}\left(\begin{array}{cc}
d_{k-1, N}^{*} & 0 \\
c_{k} & d_{k, N}^{*}
\end{array}\right),
\end{array}
$$

where $d_{k, N}$ is the exterior differential on $\Omega^{k}\left(N, E_{N}\right)$. Following a suggestion of M. Lesch, we decompose the de Rham complex $\left(\Omega_{c}^{*}\left(C_{I}(N), E\right), d_{*}\right)$ into a direct sum of subcomplexes of two types. The first type of the subcomplexes is given as follows. Let $\psi \in \Omega^{k}\left(N, E_{N}\right)$ be a coclosed eigenform of the Laplacian $\Delta_{k, N}$ on $\Omega^{k}\left(N, E_{N}\right)$ with eigenvalue $\eta>0$. We consider the following four associated pairs

$$
\begin{aligned}
& \xi_{1}:=(0, \psi) \in \Omega^{k-1}\left(N, E_{N}\right) \oplus \Omega^{k}\left(N, E_{N}\right), \\
& \xi_{2}:=(\psi, 0) \in \Omega^{k}\left(N, E_{N}\right) \oplus \Omega^{k+1}\left(N, E_{N}\right), \\
& \xi_{3}:=\left(0, d_{N} \psi / \sqrt{\eta}\right) \in \Omega^{k}\left(N, E_{N}\right) \oplus \Omega^{k+1}\left(N, E_{N}\right), \\
& \xi_{4}:=\left(d_{N} \psi / \sqrt{\eta}, 0\right) \in \Omega^{k+1}\left(N, E_{N}\right) \oplus \Omega^{k+2}\left(N, E_{N}\right) .
\end{aligned}
$$

Denote by $\left\langle\xi_{1}, \xi_{2}, \xi_{3}, \xi_{4}\right\rangle$ the span of $\xi_{1}, \ldots, \xi_{4}$. Then $C_{c}^{\infty}\left(I,\left\langle\xi_{1}, \xi_{2}, \xi_{3}, \xi_{4}\right\rangle\right)$ is invariant under $d, d^{*}$ and we obtain a subcomplex

$$
0 \rightarrow C_{c}^{\infty}\left(I,\left\langle\xi_{1}\right\rangle\right) \stackrel{d_{0}}{\rightarrow} C_{c}^{\infty}\left(I,\left\langle\xi_{2}, \xi_{3}\right\rangle\right) \stackrel{d_{1}}{\rightarrow} C_{c}^{\infty}\left(I,\left\langle\xi_{4}\right\rangle\right) \rightarrow 0
$$

where $d_{0}, d_{1}$ take the following form with respect to the chosen basis:

$$
d_{0}=\left(\begin{array}{c}
(-1)^{k} \partial_{x}+\frac{c_{k}}{x} \\
x^{-1} \sqrt{\eta}
\end{array}\right), \quad d_{1}=\left(x^{-1} \sqrt{\eta},(-1)^{k+1} \partial_{x}+\frac{c_{k+1}}{x}\right) .
$$


The associated Laplacians are of the following form

$$
\Delta_{0, \eta}:=d_{0}^{*} d_{0}=-\partial_{x}^{2}+\frac{1}{x^{2}}\left[\eta+\left(k+\frac{1}{2}-\frac{n}{2}\right)^{2}-\frac{1}{4}\right]=d_{1} d_{1}^{*}=: \Delta_{1, \eta} .
$$

with respect to the identification of $\phi=f \cdot \xi_{i} \in C_{c}^{\infty}\left(I,\left\langle\xi_{i}\right\rangle\right), i=1, \ldots, 4$, with its scalar part $f \in C_{c}^{\infty}(I)$. The subcomplexes (5.4) always come in pairs on oriented cones. The twin subcomplex is constructed by considering $\phi:=*_{N} \psi \in \Omega^{n-k}\left(N, E_{N}\right)$. Then $d_{N}^{*} \phi / \sqrt{\eta}$ is again a coclosed eigenform of the Laplacian on $\Omega^{n-k-1}\left(N, E_{N}\right)$ with eigenvalue $\eta$, and we put

$$
\begin{aligned}
& \widetilde{\xi}_{1}:=\left(0, d_{N}^{*} \phi / \sqrt{\eta}\right) \in \Omega^{n-k-2}\left(N, E_{N}\right) \oplus \Omega^{n-k-1}\left(N, E_{N}\right), \\
& \widetilde{\xi}_{2}:=\left(d_{N}^{*} \phi / \sqrt{\eta}, 0\right) \in \Omega^{n-k-1}\left(N, E_{N}\right) \oplus \Omega^{n-k}\left(N, E_{N}\right), \\
& \widetilde{\xi}_{3}:=(0, \phi) \in \Omega^{n-k-1}\left(N, E_{N}\right) \oplus \Omega^{n-k}\left(N, E_{N}\right), \\
& \widetilde{\xi}_{4}:=(\phi, 0) \in \Omega^{n-k}\left(N, E_{N}\right) \oplus \Omega^{n-k+1}\left(N, E_{N}\right) .
\end{aligned}
$$

Denote by $\left\langle\widetilde{\xi}_{1}, \widetilde{\xi}_{2}, \widetilde{\xi}_{3}, \widetilde{\xi}_{4}\right\rangle$ the vector space, spanned by these vectors. $C_{c}^{\infty}\left(I,\left\langle\widetilde{\xi}_{1}, \widetilde{\xi}_{2}, \widetilde{\xi}_{3}, \widetilde{\xi}_{4}\right\rangle\right)$ is invariant under the action of $d, d^{*}$ and we obtain a subcomplex

$$
0 \rightarrow C_{c}^{\infty}\left(I,\left\langle\widetilde{\xi_{1}}\right\rangle\right) \stackrel{\widetilde{d_{0}}}{\rightarrow} C_{c}^{\infty}\left(I,\left\langle\widetilde{\xi_{2}}, \widetilde{\xi_{3}}\right\rangle\right) \stackrel{\widetilde{d_{1}}}{\rightarrow} C_{c}^{\infty}\left(I,\left\langle\widetilde{\xi_{4}}\right\rangle\right) \rightarrow 0
$$

Computing explicitly the action of the exterior derivative (5.2) on the basis elements $\widetilde{\xi}_{i}$ we find

$$
\widetilde{d}_{0}=\left(\begin{array}{c}
(-1)^{n-k-1} \partial_{x}+\frac{c_{n-k-1}}{x} \\
x^{-1} \sqrt{\eta}
\end{array}\right), \quad \widetilde{d}_{1}=\left(x^{-1} \sqrt{\eta},(-1)^{n-k} \partial_{x}+\frac{c_{n-k}}{x}\right) .
$$

As before we compute the corresponding Laplacians and find

$$
\widetilde{\Delta}_{0, \eta}=\widetilde{\Delta}_{1, \eta}=-\partial_{x}^{2}+\frac{1}{x^{2}}\left[\eta+\left(k+\frac{1}{2}-\frac{n}{2}\right)^{2}-\frac{1}{4}\right]=\Delta_{0, \eta}=\Delta_{1, \eta},
$$

where the operators are again identified with their scalar actions.

The second type of the subcomplexes comes from the harmonic forms $\mathcal{H}^{k}\left(N, E_{N}\right)$ on $N$. Fix an orthonormal basis $\left\{u_{i}\right\}$ of $\mathcal{H}^{k}\left(N, E_{N}\right)$ and observe that any subspace $C_{c}^{\infty}\left(I,\left\langle 0 \oplus u_{i}, u_{i} \oplus 0\right\rangle\right)$ is invariant under $d, d^{*}$. Consequently we obtain a subcomplex of the de Rham complex

$$
\begin{gathered}
0 \rightarrow C_{c}^{\infty}\left(I,\left\langle 0 \oplus u_{i}^{k}\right\rangle\right) \stackrel{d_{k}^{H}}{\longrightarrow} C_{c}^{\infty}\left(I,\left\langle u_{i}^{k} \oplus 0\right\rangle\right) \rightarrow 0, \\
d_{k}^{H}=(-1)^{k} \partial_{x}+\frac{c_{k}}{x}
\end{gathered}
$$


where the action of $d_{k}^{H}$ is identified with its scalar action, as before. The Laplacians of the complex are given by

$$
\begin{aligned}
& H_{0}^{k}:=\left(d_{k}^{H}\right)^{*} d_{k}^{H}=-\partial_{x}^{2}+\frac{1}{x^{2}}\left(\left(\frac{(n-1)}{2}-k\right)^{2}-\frac{1}{4}\right), \\
& H_{1}^{k}:=d_{k}^{H}\left(d_{k}^{H}\right)^{*}=-\partial_{x}^{2}+\frac{1}{x^{2}}\left(\left(\frac{(n+1)}{2}-k\right)^{2}-\frac{1}{4}\right) .
\end{aligned}
$$

5.2. The Relative Ideal Boundary Conditions. By (2.9) the analytic torsion with respect to absolute or relative boundary conditions coincide. We will work with relative boundary conditions.

Let $d_{k, \max }$ denote the maximal extension of $d_{k}$ in $L^{2}\left(\Omega_{c}^{*}\left(C_{I}(N), E\right), g, h^{E}\right)$. Furthermore, let $d_{k, \text { min }}$ denote the graph closure of $d_{k}$ in $L^{2}\left(\Omega_{c}^{*}\left(C_{I}(N), E\right), g, h^{E}\right)$. Then we have $\mathscr{D}\left(d_{k, \text { min }}\right) \subset \mathscr{D}\left(d_{k, \text { max }}\right)$, where both spaces are the Hilbert spaces equipped with the graph-norm. Despite the fact that the differential $d_{k}$ is not elliptic, there is still a well-defined trace on its maximal domain by the trace theorem of Paquet [PAQ82].

Theorem 5.1. [PAQ82, Theorem 1.9] Let $\left(X, g^{X}\right)$ be a compact oriented Riemannian manifold with isolated conical singularities and with smooth boundary $\partial X$. Let $\iota: \partial X \hookrightarrow X$ be the natural inclusion. Let $\left(E, \nabla, h^{E}\right)$ be a flat complex Hermitian vector bundle over $X$ and $\left(E_{\partial X}, \nabla_{\partial X}, h_{\partial X}\right)$ its restriction to the boundary. Then the pullback $\iota^{*}: \Omega^{k}(X, E) \rightarrow \Omega^{k}\left(\partial X, E_{\partial X}\right)$ with $\Omega^{k}\left(\partial X, E_{\partial X}\right)=\{0\}$ for $k=\operatorname{dim} X$, extends continuously to a linear surjective map

$$
\iota^{*}: \mathscr{D}\left(d_{k, \max }\right) \rightarrow H^{-1 / 2}\left(d_{k, \partial X}\right),
$$

where $d_{k, \partial X}$ is the differential on $\Omega^{k}\left(\partial X, E_{\partial X}\right), H^{-1 / 2}\left(\Omega^{k}\left(\partial X, E_{\partial X}\right)\right)$ the $(-1 / 2)-t h$ Sobolev space on $\partial X$ and

$$
H^{-1 / 2}\left(d_{k, \partial X}\right):=\left\{\omega \in H^{-1 / 2}\left(\Omega^{k}\left(\partial X, E_{\partial X}\right)\right) \mid d_{k, \partial X} \omega \in H^{-1 / 2}\left(\Omega^{k+1}\left(\partial X, E_{\partial X}\right)\right)\right\},
$$

is a Hilbert space with respect to the graph-norm.

Remark 5.2. The trace theorem [PAQ82, Theorem 1.9] is stated for the untwisted case on compact (non-singular) Riemannian manifolds. Extension to flat Hermitian vector bundles is straightforward. Moreover, the analysis localizes to an open neighborhood of the boundary $\partial X$, so the trace theorem carries over to compact Riemannian manifolds with singular structure away from $\partial X$.

Fix the relative extension of the Laplacian, induced by $d_{k, \min }^{*}$

$$
\Delta_{k}^{\mathrm{rel}}:=d_{k, \min }^{*} d_{k, \min }+d_{k-1, \min } d_{k-1, \min }^{*}
$$

The minimal extension $d_{k, \text { min }}$ is defined as the graph closure of the de Rham differential $d_{k}$ on $\Omega_{c}^{*}\left(C_{I}(N), E\right)$ in $L^{2}\left(\Omega^{*}\left(C_{I}(N), E\right), g, h^{E}\right)$. Hence, Theorem 5.1 implies

$$
\begin{aligned}
& \mathscr{D}\left(d_{k, \text { min }}\right) \subseteq\left\{\omega \in \mathscr{D}\left(d_{k, \text { max }}\right) \mid \iota^{*} \omega=0\right\}, \\
& \mathscr{D}\left(\Delta_{k}^{\mathrm{rel}}\right) \subseteq\left\{\omega \in \mathscr{D}\left(\Delta_{k, \text { max }}\right) \mid \iota^{*} \omega=0, \iota^{*}\left(d_{k-1}^{*} \omega\right)=0\right\} .
\end{aligned}
$$


By the Hodge decomposition of $\Omega^{*}\left(N, E_{N}\right)$, the de Rham complex $\left(\Omega_{c}^{*}\left(C_{I}(N)\right)\right.$, d) decomposes completely into subcomplexes of the three types (5.4), (5.7) and (5.9). It has been observed in [VER09A, Theorem 3.5] that in each degree $k$ this induces a compatible decomposition for the relative extension of the Laplacian. In the classical language of [WEI80] we have a decomposition into reducing subspaces of the Laplacians. Hence the Laplacians $\Delta_{k}^{\text {rel }}$ induce self-adjoint relative extensions of the Laplacians $\Delta_{j}^{\psi}, \Delta_{j}^{\phi}$, $j=0,1$, and $H_{0}^{k}, H_{1}^{k}$.

In order to discuss the corresponding relative boundary conditions explicitly, note that by the classical theory of linear differential equations for any element $f$ of $\mathscr{D}\left(\Delta_{j, \text { max }}^{\psi}\right), \mathscr{D}\left(\Delta_{j, \text { max }}^{\phi}\right), j=0,2$, or $\mathscr{D}\left(H_{i, \text { max }}^{k}\right), i=0,1, f$ and its derivative $f^{\prime}$ are both locally absolutely continuous in $I$ with well-defined values at $x \in \partial I$. More precisely at $x=1$ in case $I=(0,1]$, and $x \in\{\epsilon, 1\}$ in case $I=[\epsilon, 1]$. Hence the following boundary conditions are well-defined

$$
B_{N}^{k}(x) f:=f^{\prime}(x)+(-1)^{k+1} c_{k} f(x), \quad B_{D}(x) f:=f(x), \quad x \in \partial I .
$$

In case of $I=(0,1]$ boundary conditions at $x=0$ need to be imposed. By the wellknown analysis, compare [BRSE88], [CHE79B] and the basic discussion of the second author [VER09B], any solution $f \in L^{2}(0,1)$ to

$$
-\frac{d^{2} f}{d x^{2}}+\frac{1}{x^{2}}\left(\nu^{2}-\frac{1}{4}\right) f=g \in L^{2}(0,1)
$$

admits an asymptotic expansion of the form

$$
f(x) \sim \begin{cases}c_{1}(f) \sqrt{x}+c_{2}(f) \sqrt{x} \log (x)+O\left(x^{3 / 2}\right), & \nu=0, \\ c_{1}(f) x^{\nu+1 / 2}+c_{2}(f) x^{-\nu+1 / 2}+O\left(x^{3 / 2}\right), & \nu \in(0,1), \quad x \rightarrow 0, \\ O\left(x^{3 / 2}\right), & \nu \geq 1,\end{cases}
$$

where the coefficients $c_{1}(f)$ and $c_{2}(f)$ depend only on $f$. Consequently the following boundary conditions at $x=0$ are well-defined

$$
B_{N}(0) f:=\left\{\begin{array}{ll}
c_{1}(f), & \nu \in[0,1), \\
0, & \nu \geq 1,
\end{array} \quad B_{D}(0) f:= \begin{cases}c_{2}(f), & \nu \in[0,1) \\
0, & \nu \geq 1\end{cases}\right.
$$

Proposition 5.3. Let $\Delta_{j, \eta}, \widetilde{\Delta}_{j, \eta}, j=0,1$, be the Laplacians of the pair of subcomplexes (5.4) and (5.7), and let $H_{0}^{k}, H_{1}^{k}$, be the Laplacians of the subcomplex (5.9). The domains of their relative self-adjoint extensions are given as follows. For $I=[\epsilon, 1]$

$$
\begin{aligned}
& \mathscr{D}\left(\Delta_{0, \eta}^{\mathrm{rel}}\right)=\left\{f \in \mathscr{D}\left(\Delta_{0, \eta}^{\max }\right) \mid B_{D}(\epsilon) f=0, B_{D}(1) f=0\right\}=\mathscr{D}\left(\widetilde{\Delta}_{0, \eta}^{\mathrm{rel}}\right), \\
& \mathscr{D}\left(\Delta_{1, \eta}^{\mathrm{rel}}\right)=\left\{f \in \mathscr{D}\left(\Delta_{1, \eta}^{\max }\right) \mid B_{N}^{k+1}(\epsilon) f=0, B_{N}^{k+1}(1) f=0\right\}, \\
& \mathscr{D}\left(\widetilde{\Delta}_{1, \eta}^{\mathrm{rel}}\right)=\left\{f \in \mathscr{D}\left(\widetilde{\Delta}_{1, \eta}^{\max }\right) \mid B_{N}^{n-k}(\epsilon) f=0, B_{N}^{n-k}(1) f=0\right\}, \\
& \mathscr{D}\left(H_{1, \mathrm{rel}}^{k}\right)=\left\{f \in \mathscr{D}\left(H_{1, \text { max }}^{k}\right) \mid B_{N}^{k}(\epsilon) f=0, B_{N}^{k}(1) f=0\right\}, \\
& \mathscr{D}\left(H_{0, \mathrm{rel}}^{k}\right)=\left\{f \in \mathscr{D}\left(H_{0, \text { max }}^{k}\right) \mid B_{D}(\epsilon) f=0, B_{D}(1) f=0\right\} .
\end{aligned}
$$


For $I=(0,1]$ the domains are given by

$$
\begin{aligned}
& \mathscr{D}\left(\Delta_{0, \eta}^{\mathrm{rel}}\right)=\left\{f \in \mathscr{D}\left(\Delta_{0, \eta}^{\max }\right) \mid B_{D}(0) f=0, B_{D}(1) f=0\right\}=\mathscr{D}\left(\widetilde{\Delta}_{0, \eta}^{\mathrm{rel}}\right), \\
& \mathscr{D}\left(\Delta_{1, \eta}^{\mathrm{rel}}\right)=\left\{f \in \mathscr{D}\left(\left(\Delta_{1, \eta}^{\max }\right) \mid B_{D}(0) f=0, B_{N}^{k+1}(1) f=0\right\},\right. \\
& \mathscr{D}\left(\widetilde{\Delta}_{1, \eta}^{\mathrm{rel}}\right)=\left\{f \in \mathscr{D}\left(\widetilde{\Delta}_{1, \eta}^{\max }\right) \mid B_{D}(0) f=0, B_{N}^{n-k}(1) f=0\right\}, \\
& \mathscr{D}\left(H_{1, \mathrm{rel}}^{k}\right)=\left\{f \in \mathscr{D}\left(H_{1, \text { max }}^{k}\right) \mid B_{N}(0) f=0, B_{N}^{k}(1) f=0\right\}, \\
& \mathscr{D}\left(H_{0, \mathrm{rel}}^{k}\right)=\left\{f \in \mathscr{D}\left(H_{0, \text { max }}^{k}\right) \mid B_{D}(0) f=0, B_{D}(1) f=0\right\} .
\end{aligned}
$$

Proof. The choice of the boundary conditions at $x=1$ in case $I=(0,1]$, and at $x \in\{\epsilon, 1\}$ in case $I=[\epsilon, 1]$, follows for the individual relative self-adjoint extensions from (5.13), the explicit form of the exterior differentials (5.2) and the fact that for any $x \in \partial I$ and the inclusion $\iota_{x}:\{x\} \times N \hookrightarrow C_{I}(N)$, we have $\iota_{x}^{*}\left(f_{k-1}, f_{k}\right)=f_{k}(x)$ for any $\left(f_{k-1}, f_{k}\right) \in \mathscr{D}\left(\Delta_{\max }\right)$ with $f_{k}$ continuous at $x$. The boundary conditions at $x=0$ in case $I=(0,1]$ have been determined in [VER09B, Corollary 2.14] and [VER09A, Proposition 3.6 and 3.7].

5.3. The Difference of Analytic Torsion for the Truncated and the Full Cone. Let $\left(N^{n}, g^{N}\right)$ be a closed Riemannian manifold of even dimension. Let $C(N):=(0,1] \times$ $N$, equipped with the metric $g=d x^{2} \oplus x^{2} g^{N}$ and for $\varepsilon>0$ let $C_{\varepsilon}(N):=[\varepsilon, 1] \times N$, equipped with the same metric. Let $\Delta_{k}^{\text {rel }}$ and $\Delta_{k, \epsilon}^{\text {rel }}$ denote the Laplacians with relative boundary conditions on $k$-forms associated to $(C(N), g)$ and $\left(C_{\epsilon}(N), g\right)$, respectively. Put

$$
T(\epsilon, s):=\frac{1}{2} \sum_{k=1}^{\operatorname{dim} C(N)}(-1)^{k} \cdot k \cdot\left(\zeta\left(s, \Delta_{k, \epsilon}^{\mathrm{rel}}\right)-\zeta\left(s, \Delta_{k}^{\mathrm{rel}}\right)\right) .
$$

$T(\epsilon, s)$ is related to the scalar analytic torsions of $(C(N), g)$ and $\left(C_{\epsilon}(N), g\right)$ by

$$
T^{\prime}(\epsilon, 0)=\log T\left(C_{\epsilon}(N), E, g\right)-\log T(C(N), E, g) .
$$

Consider the decomposition of the de Rham complex as described in Section 5.1. For each fixed degree $k$, the subcomplexes (5.4) and (5.7) are determined by a coclosed eigenform $\psi \in \Omega^{k}\left(N, E_{N}\right)$ of the Laplacian $\Delta_{k, N}$ with eigenvalue $\eta>0$. For $k=$ $0, \ldots, n+1$ let

$$
E_{k}:=\operatorname{Spec}\left(\Delta_{k, c c l, N}\right) \backslash\{0\}
$$

For $\eta \in E_{k}$ the relative boundary conditions for the Laplacians $\Delta_{j, \eta}, \widetilde{\Delta}_{j, \eta}, j=0$, 1 , of the subcomplex-pair (5.4) and (5.7), and the Laplacians $H_{0}^{k}, H_{1}^{k}$, of the subcomplex (5.9) are discussed in Proposition 5.3. Here we distinguish operators on $\left(C_{\epsilon}(N), g\right)$ by an additional $\epsilon$-subscript.

Definition 5.4. Denote by $\mathrm{m}(\eta)$ the multiplicity of $\eta \in E_{k}$. For $\operatorname{Re}(s) \gg 0$ put

$$
\begin{aligned}
& \zeta_{k, H}(s, \epsilon):=\operatorname{dim} H^{k}\left(N, E_{N}\right)\left(\zeta\left(s, H_{0, \epsilon, \text { rel }}^{k}\right)-\zeta\left(s, H_{0, \text { rel }}^{k}\right)\right), \\
& \zeta_{k}(s, \epsilon):=\sum_{\eta \in E_{k}} \mathrm{~m}(\eta)\left(\zeta\left(s, \Delta_{1, \eta, \epsilon}^{\text {rel }}\right)-\zeta\left(s, \widetilde{\Delta}_{1, \eta, \epsilon}^{\text {rel }}\right)\right)-\left(\zeta\left(s, \Delta_{1, \eta}^{\text {rel }}\right)-\zeta\left(s, \widetilde{\Delta}_{1, \eta}^{\text {rel }}\right)\right) .
\end{aligned}
$$


We note that $T(\epsilon, s)$ can be expressed in terms of $\zeta_{k, H}(s, \epsilon)$ and $\zeta_{k}(s, \epsilon)$ as follows

$$
T(\epsilon, s)=\frac{1}{2} \sum_{k=0}^{n / 2-1}(-1)^{k} \zeta_{k}(s, \epsilon)+\frac{1}{2} \sum_{k=0}^{n}(-1)^{k+1} \zeta_{k, H}(s, \epsilon), \operatorname{Re}(s) \gg 0
$$

(see [Ver09A, (4.3), (4.4)]). Evaluation of $\zeta_{k}^{\prime}(0, \epsilon)$ requires application of the double summation method, which has been introduced by Spreafico in [Spr05], [Spr06] and was applied by the second named author in [VER09A], see Theorem 2.6, to derive the general formula for analytic torsion of a bounded cone in. Evaluation of $\zeta_{k, H}^{\prime}(0, \epsilon)$ reduces to an explicit computation of finitely many zeta-determinants and application of [LES98]. We begin with the evaluation of $\zeta_{k}^{\prime}(0, \epsilon)$ for each fixed degree $k$ along the lines of [Ver09A, Section 6].

Proposition 5.5. For $c>0$ let

$$
\Lambda_{c}:=\{\lambda \in \mathbb{C}:|\arg (\lambda-c)|=\pi / 4\}
$$

and assume that $\Lambda_{c}$ is oriented counter-clockwise. Put

$$
\alpha_{k}:=\frac{(n-1)}{2}-k, \quad \nu(\eta):=\sqrt{\eta+\alpha_{k}^{2}}, \eta \in \text { Spec. } \Delta_{k, c c l, N} \backslash\{0\}
$$

Let $c(\eta)=c_{0} /\left(2 \nu(\eta)^{2}\right)$, where $c_{0}>0$ is a fixed positive number, smaller than the lowest non-zero eigenvalue of $\Delta_{*}^{\mathrm{rel}}$ and $\Delta_{*, \epsilon}^{\mathrm{rel}}$, such that $c(\eta)<1$ for all $\eta \in E_{k}$. Then $\zeta_{k}(s, \epsilon)$ admits the following integral representation for $\operatorname{Re}(s) \gg 0$

$$
\zeta_{k}(s, \epsilon)=\sum_{\eta \in E_{k}} \mathrm{~m}(\eta) \nu(\eta)^{-2 s} \frac{s^{2}}{\Gamma(s+1)} \int_{0}^{\infty} \frac{t^{s-1}}{2 \pi i} \int_{\wedge_{c(\eta)}} \frac{e^{-\lambda t}}{-\lambda} t_{\eta, \epsilon}^{k}(\lambda) d \lambda d t
$$

where $t_{\eta, \epsilon}^{k}(\lambda)$ is defined in terms of zeta-determinants by

$$
\begin{aligned}
t_{\eta, \epsilon}^{k}(\lambda)=- & \log \frac{\operatorname{det}_{\zeta}\left(\Delta_{1, \eta, \epsilon}^{\mathrm{rel}}-\nu(\eta)^{2} \lambda\right)}{\operatorname{det}\left(\Delta_{1, \eta, \epsilon}^{\mathrm{rel}}\right)}+\log \frac{\operatorname{det}_{\zeta}\left(\widetilde{\Delta}_{1, \eta, \epsilon}^{\mathrm{rel}}-\nu(\eta)^{2} \lambda\right)}{\operatorname{det}\left(\widetilde{\Delta}_{1, \eta, \epsilon}^{\mathrm{rel}}\right)} \\
& +\log \frac{\operatorname{det}_{\zeta}\left(\Delta_{1, \eta}^{\mathrm{rel}}-\nu(\eta)^{2} \lambda\right)}{\operatorname{det}\left(\Delta_{1, \eta}^{\mathrm{rel}}\right)}-\log \frac{\operatorname{det}_{\zeta}\left(\widetilde{\Delta}_{1, \eta}^{\mathrm{rel}}-\nu(\eta)^{2} \lambda\right)}{\operatorname{det}\left(\widetilde{\Delta}_{1, \eta}^{\mathrm{rel}}\right)} .
\end{aligned}
$$

and $\log$ denotes the main branch of the logarithm.

Proof. Recall that the spectrum used to define $\zeta_{k}(s, \epsilon)$ is the union of the spectra for the Laplacians $\Delta_{1, \eta, \epsilon}^{\text {rel }}, \Delta_{1, \eta}^{\text {rel }}$ and $\widetilde{\Delta}_{1, \eta, \epsilon}^{\text {rel }}, \widetilde{\Delta}_{1, \eta}^{\text {rel }}$, where $\eta$ runs over $E_{k}$. For any choice

$$
L(\eta) \in\left\{\Delta_{1, \eta, \epsilon}^{\mathrm{rel}}, \Delta_{1, \eta}^{\mathrm{rel}}, \widetilde{\Delta}_{1, \eta, \epsilon}^{\mathrm{rel}}, \widetilde{\Delta}_{1, \eta}^{\mathrm{rel}}\right\}, \eta \in E_{k},
$$

the spectrum $\operatorname{Spec} L(\eta) \subset \mathbb{R}^{+}$is strictly positive. Indeed, Spec $L(\eta)$ is contained in the spectrum of the non-negative Laplace operator on the truncated or full cone, and its zero eigenvalues arise in both cases only from harmonic forms $H^{*}\left(N, E_{N}\right)$. We note 
that the resolvent of $L(\eta)$ is a trace class operator [LES98], and from Definition 5.4 we infer for $\operatorname{Re}(s) \gg 0$

$$
\zeta_{k}(s, \epsilon)=\sum_{\eta \in E_{k}} \mathrm{~m}(\eta) \nu(\eta)^{-2 s} \frac{1}{\Gamma(s)} \int_{0}^{\infty} t^{s-1} \frac{1}{2 \pi i} \int_{\wedge_{c(\eta)}} e^{-\lambda t} h_{\eta, \epsilon}^{k}(\lambda) d \lambda d t
$$

where

$$
\begin{aligned}
h_{\eta, \epsilon}^{k}(\lambda) & =\operatorname{Tr}\left(\lambda-\nu(\eta)^{-2} \Delta_{2, \epsilon, \mathrm{rel}}^{\psi(\eta)}\right)^{-1}-\operatorname{Tr}\left(\lambda-\nu(\eta)^{-2} \Delta_{2, \epsilon, \mathrm{rel}}^{\phi(\eta)}\right)^{-1} \\
& -\operatorname{Tr}\left(\lambda-\nu(\eta)^{-2} \Delta_{1, \eta}^{\mathrm{rel}}\right)^{-1}+\operatorname{Tr}\left(\lambda-\nu(\eta)^{-2} \widetilde{\Delta}_{1, \eta}^{\mathrm{rel}}\right)^{-1} .
\end{aligned}
$$

For any choice of

$$
L(\eta) \in\left\{\Delta_{1, \eta, \epsilon}^{\mathrm{rel}}, \Delta_{1, \eta}^{\mathrm{rel}}, \widetilde{\Delta}_{1, \eta, \epsilon}^{\mathrm{rel}}, \widetilde{\Delta}_{1, \eta}^{\mathrm{rel}}\right\}, \eta \in E_{k},
$$

we find by [LEs98, Proposition 4.6] that, enumerating Spec $L(\eta)=\left\{\lambda_{i}\right\}_{i=1}^{\infty}$ in increasing order, the series

$$
\log \frac{\operatorname{det}_{\zeta}\left(L(\eta)-\nu(\eta)^{2} \lambda\right)}{\operatorname{det}_{\zeta} L(\eta)}=\sum_{i=1}^{\infty} \log \left(1-\frac{\nu(\eta)^{2} \lambda}{\lambda_{i}}\right) .
$$

converges and by the choice of the logarithm branch is holomorphic in $\lambda \in \mathbb{C} \backslash\{x \in \mathbb{R} \mid$ $x>c(\eta)\}$. Moreover,

$$
\operatorname{Tr}\left(\frac{L(\eta)}{\nu(\eta)^{2}}-\lambda\right)^{-1}=-\frac{d}{d \lambda} \log \frac{\operatorname{det}_{\zeta}\left(L(\eta)-\nu(\eta)^{2} \lambda\right)}{\operatorname{det}_{\zeta} L(\eta)} .
$$

By the definition of $c(\eta)>0,(5.23)$ is holomorphic in an open neighborhood of the contour $\Lambda_{c(\eta)}$, and so we may integrate (5.22) by parts first in $\lambda$ then in $t$, and obtain

$$
\begin{aligned}
\zeta_{k}(s, \epsilon) & =\sum_{\eta \in E_{k}} \mathrm{~m}(\eta) \nu(\eta)^{-2 s} \frac{1}{\Gamma(s)} \int_{0}^{\infty} t^{s-1} \frac{1}{2 \pi i} \int_{\wedge_{c(\eta)}} e^{-\lambda t} h_{\eta, \epsilon}^{k}(\lambda) d \lambda d t \\
& =\sum_{\eta \in E_{k}} \mathrm{~m}(\eta) \nu(\eta)^{-2 s} \frac{s^{2}}{\Gamma(s+1)} \int_{0}^{\infty} t^{s-1} \frac{1}{2 \pi i} \int_{\wedge_{c(\eta)}} \frac{e^{-\lambda t}}{-\lambda} t_{\eta, \epsilon}^{k}(\lambda) d \lambda d t
\end{aligned}
$$

where

$$
\begin{aligned}
t_{\eta, \epsilon}^{k}(\lambda)=- & \log \frac{\operatorname{det}_{\zeta}\left(\Delta_{1, \eta, \epsilon}^{\mathrm{rel}}-\nu(\eta)^{2} \lambda\right)}{\operatorname{det}\left(\Delta_{1, \eta, \epsilon}^{\mathrm{rel}}\right)}+\log \frac{\operatorname{det}_{\zeta}\left(\widetilde{\Delta}_{1, \eta, \epsilon}^{\mathrm{rel}}-\nu(\eta)^{2} \lambda\right)}{\operatorname{det}\left(\widetilde{\Delta}_{1, \eta, \epsilon}^{\mathrm{rel}}\right)} \\
& +\log \frac{\operatorname{det}_{\zeta}\left(\Delta_{1, \eta}^{\mathrm{rel}}-\nu(\eta)^{2} \lambda\right)}{\operatorname{det}\left(\Delta_{1, \eta}^{\mathrm{rel}}\right)}-\log \frac{\operatorname{det}_{\zeta}\left(\widetilde{\Delta}_{1, \eta}^{\mathrm{rel}}-\nu(\eta)^{2} \lambda\right)}{\operatorname{det}\left(\widetilde{\Delta}_{1, \eta}^{\mathrm{rel}}\right)}
\end{aligned}
$$


Lemma 5.6. For any $\nu>0$ and $z \in \mathbb{C}$ we have

$$
\begin{aligned}
& \frac{\operatorname{det}_{\zeta}\left(\Delta_{1, \eta}^{\mathrm{rel}}+\nu^{2} z^{2}\right)}{\operatorname{det}\left(\Delta_{1, \eta}^{\mathrm{rel}}\right)}=\frac{2^{\nu} \Gamma(\nu)}{(\nu z)^{\nu}\left(1+\alpha_{k} / \nu\right)}\left(\nu z I_{\nu}^{\prime}(\nu z)+\alpha_{k} I_{\nu}(\nu z)\right) \\
& \frac{\operatorname{det}_{\zeta}\left(\widetilde{\Delta}_{1, \eta}^{\mathrm{rel}}+\nu^{2} z^{2}\right)}{\operatorname{det}\left(\widetilde{\Delta}_{1, \eta}^{\mathrm{rel}}\right)}=\frac{2^{\nu} \Gamma(\nu)}{(\nu z)^{\nu}\left(1-\alpha_{k} / \nu\right)}\left(\nu z I_{\nu}^{\prime}(\nu z)-\alpha_{k} I_{\nu}(\nu z)\right) . \\
& \frac{\operatorname{det}_{\zeta}\left(\Delta_{1, \eta, \epsilon}^{\mathrm{rel}}+\nu^{2} z^{2}\right)}{\operatorname{det}\left(\Delta_{1, \eta, \epsilon}^{\mathrm{rel}}\right)}=\frac{\left(\nu z I_{\nu}^{\prime}(\nu z)+\alpha_{k} I_{\nu}(\nu z)\right)\left(\nu z \epsilon K_{\nu}^{\prime}(\nu z \epsilon)+\alpha_{k} K_{\nu}(\nu z \epsilon)\right)}{\left(\nu^{2}-\alpha_{k}^{2}\right)\left(\epsilon^{\nu}-\epsilon^{-\nu}\right)} \\
& \times 2 \nu \sqrt{\epsilon}\left(1-\frac{\nu z K_{\nu}^{\prime}(\nu z)+\alpha_{k} K_{\nu}(\nu z)}{\nu z I_{\nu}^{\prime}(\nu z)+\alpha_{k} I_{\nu}(\nu z)} \cdot \frac{\nu z \epsilon I_{\nu}^{\prime}(\nu z \epsilon)+\alpha_{k} I_{\nu}(\nu z \epsilon)}{\nu z \epsilon K_{\nu}^{\prime}(\nu z \epsilon)+\alpha_{k} K_{\nu}(\nu z \epsilon)}\right), \\
& \frac{\operatorname{det}_{\zeta}\left(\widetilde{\Delta}_{1, \eta, \epsilon}^{\mathrm{rel}}+\nu^{2} z^{2}\right)}{\operatorname{det}\left(\widetilde{\Delta}_{1, \eta, \epsilon}^{\mathrm{rel}}\right)}=\frac{\left(\nu z I_{\nu}^{\prime}(\nu z)-\alpha_{k} I_{\nu}(\nu z)\right)\left(\nu z \epsilon K_{\nu}^{\prime}(\nu z \epsilon)-\alpha_{k} K_{\nu}(\nu z \epsilon)\right)}{\left(\nu^{2}-\alpha_{k}^{2}\right)\left(\epsilon^{\nu}-\epsilon^{-\nu}\right)} \\
& \times 2 \nu \sqrt{\epsilon}\left(1-\frac{\nu z K_{\nu}^{\prime}(\nu z)-\alpha_{k} K_{\nu}(\nu z)}{\nu z I_{\nu}^{\prime}(\nu z)-\alpha_{k} I_{\nu}(\nu z)} \cdot \frac{\nu z \epsilon I_{\nu}^{\prime}(\nu z \epsilon)-\alpha_{k} I_{\nu}(\nu z \epsilon)}{\nu z \epsilon K_{\nu}^{\prime}(\nu z \epsilon)-\alpha_{k} K_{\nu}(\nu z \epsilon)}\right)
\end{aligned}
$$

Proof. We evaluate the zeta-determinants using their explicit relation with the normalized solutions of the operators, established by Lesch in [LEs98, Theorem 1.2]. The first two equations have been evaluated in [VER09A, Corollary 6.3]:

$$
\begin{aligned}
& \frac{\operatorname{det}_{\zeta}\left(\Delta_{1, \eta}^{\mathrm{rel}}+\nu^{2} z^{2}\right)}{\operatorname{det}\left(\Delta_{1, \eta}^{\mathrm{rel}}\right)}=\frac{2^{\nu} \Gamma(\nu)}{(\nu z)^{\nu}\left(1+\alpha_{k} / \nu\right)}\left(\nu z I_{\nu}^{\prime}(\nu z)+\alpha_{k} I_{\nu}(\nu z)\right), \\
& \frac{\operatorname{det}_{\zeta}\left(\widetilde{\Delta}_{1, \eta}^{\mathrm{rel}}+\nu^{2} z^{2}\right)}{\operatorname{det}\left(\widetilde{\Delta}_{1, \eta}^{\mathrm{rel}}\right)}=\frac{2^{\nu} \Gamma(\nu)}{(\nu z)^{\nu}\left(1-\alpha_{k} / \nu\right)}\left(\nu z I_{\nu}^{\prime}(\nu z)-\alpha_{k} I_{\nu}(\nu z)\right) .
\end{aligned}
$$

In order to evaluate zeta determinants of $\Delta_{1, \eta, \epsilon}^{\text {rel }}$ and $\widetilde{\Delta}_{1, \eta, \epsilon}^{\text {rel }}$, consider solutions $f_{\psi, \nu}(\cdot, z)$ and $f_{\phi, \nu}(\cdot, z)$ of $\left(\Delta_{1, \eta, \epsilon}^{\text {rel }}+z^{2}\right) u=0$ and $\left(\widetilde{\Delta}_{1, \eta, \epsilon}^{\text {rel }}+z^{2}\right) v=0$, respectively, normalized at $x=1$. By definition (see [LES98, (1.38a), (1.38b)] these are solutions of the respective operators, satisfying relative boundary conditions at $x=1$ and are normalized by $f_{\psi, \nu}(1, z)=1$ and $f_{\phi, \nu}(1, z)=1$, i.e.

$$
\begin{aligned}
& \left(\Delta_{1, \eta, \epsilon}+z^{2}\right) f_{\psi, \nu}(\cdot, z)=0, \quad f_{\psi, \nu}^{\prime}(1, z)+(-1)^{k} c_{k+1} f_{\psi, \nu}(1, z)=0, \quad f_{\psi, \nu}(\cdot, z)=1, \\
& \left(\widetilde{\Delta}_{1, \eta, \epsilon}+z^{2}\right) f_{\phi, \nu}(\cdot, z)=0, \quad f_{\phi, \nu}^{\prime}(1, z)+(-1)^{n-k+1} c_{n-k} f_{\phi, \nu}(1, z)=0, \quad f_{\phi, \nu}(\cdot, z)=1 \text {. }
\end{aligned}
$$


Normalized solutions are uniquely determined and explicit computations lead to the following expressions

$$
\begin{aligned}
& f_{\psi, \nu}(x, z)=\left(z I_{\nu}^{\prime}(z)+\alpha_{k} I_{\nu}(z)\right) \sqrt{x} K_{\nu}(z x)-\left(z K_{\nu}^{\prime}(z)+\alpha_{k} K_{\nu}(z)\right) \sqrt{x} I_{\nu}(z x), \\
& f_{\phi, \nu}(x, z)=\left(z I_{\nu}^{\prime}(z)-\alpha_{k} I_{\nu}(z)\right) \sqrt{x} K_{\nu}(z x)-\left(z K_{\nu}^{\prime}(z)-\alpha_{k} K_{\nu}(z)\right) \sqrt{x} I_{\nu}(z x), \\
& f_{\psi, \nu}(x, 0)=\frac{1}{2 \nu}\left(\nu-\alpha_{k}\right) x^{\nu+1 / 2}+\frac{1}{2 \nu}\left(\nu+\alpha_{k}\right) x^{-\nu+1 / 2}, \\
& f_{\phi, \nu}(x, 0)=\frac{1}{2 \nu}\left(\nu+\alpha_{k}\right) x^{\nu+1 / 2}+\frac{1}{2 \nu}\left(\nu-\alpha_{k}\right) x^{-\nu+1 / 2},
\end{aligned}
$$

where we use

$$
K_{\nu}(z) I_{\nu}^{\prime}(z)-K_{\nu}^{\prime}(z) I_{\nu}(z)=\frac{1}{z}
$$

In view of [LES98, Theorem 1.2] we find

$$
\begin{aligned}
& \frac{\operatorname{det}_{\zeta}\left(\Delta_{1, \eta, \epsilon}^{\mathrm{rel}}+\nu^{2} z^{2}\right)}{\operatorname{det}\left(\Delta_{1, \eta, \epsilon}^{\mathrm{rel}}\right)}=\frac{f_{\psi, \nu}^{\prime}(\epsilon, \nu z)+(-1)^{k} c_{k+1} f_{\psi, \nu}(\epsilon, \nu z)}{f_{\psi, \nu}^{\prime}(\epsilon, 0)+(-1)^{k} c_{k+1} f_{\psi, \nu}(\epsilon, 0)}, \\
& \frac{\operatorname{det}_{\zeta}\left(\widetilde{\Delta}_{1, \eta, \epsilon}^{\mathrm{rel}}+\nu^{2} z^{2}\right)}{\operatorname{det}\left(\widetilde{\Delta}_{1, \eta, \epsilon}^{\mathrm{rel}}\right)}=\frac{f_{\phi, \nu}^{\prime}(\epsilon, \nu z)+(-1)^{k} c_{k+1} f_{\phi, \nu}(\epsilon, \nu z)}{f_{\phi, \nu}^{\prime}(\epsilon, 0)+(-1)^{k} c_{k+1} f_{\phi, \nu}(\epsilon, 0)} .
\end{aligned}
$$

We note that in the non-singular case this is due to Burghelea-Friedlander-Kappeler in [BFK95]. Plugging in the expressions (5.29) we obtain the lemma.

In particular, applying Lemma 5.6 several cancellations lead to a representation of $t_{\eta, \epsilon}^{k}(\lambda)$ in terms of Bessel functions with $\nu \equiv \nu(\eta)$ and $z=\sqrt{-\lambda}$, where we use the main branch of logarithm in $\mathbb{C} \backslash \mathbb{R}^{-}$, extended by continuity to one of the of the cut

$$
\begin{aligned}
t_{\eta, \epsilon}^{k}(\lambda)= & -\log \left(\nu z \epsilon K_{\nu}^{\prime}(\nu z \epsilon)+\alpha_{k} K_{\nu}(\nu z \epsilon)\right)-\log \left(1+\frac{\alpha_{k}}{\nu}\right) \\
& +\log \left(\nu z \epsilon K_{\nu}^{\prime}(\nu z \epsilon)-\alpha_{k} K_{\nu}(\nu z \epsilon)\right)+\log \left(1-\frac{\alpha_{k}}{\nu}\right) \\
& -\log \left(1-\frac{\nu z K_{\nu}^{\prime}(\nu z)+\alpha_{k} K_{\nu}(\nu z)}{\nu z I_{\nu}^{\prime}(\nu z)+\alpha_{k} I_{\nu}(\nu z)} \cdot \frac{\nu z \epsilon I_{\nu}^{\prime}(\nu z \epsilon)+\alpha_{k} I_{\nu}(\nu z \epsilon)}{\nu z \epsilon K_{\nu}^{\prime}(\nu z \epsilon)+\alpha_{k} K_{\nu}(\nu z \epsilon)}\right) \\
& +\log \left(1-\frac{\nu z K_{\nu}^{\prime}(\nu z)-\alpha_{k} K_{\nu}(\nu z)}{\nu z I_{\nu}^{\prime}(\nu z)-\alpha_{k} I_{\nu}(\nu z)} \cdot \frac{\nu z \epsilon I_{\nu}^{\prime}(\nu z \epsilon)-\alpha_{k} I_{\nu}(\nu z \epsilon)}{\nu z \epsilon K_{\nu}^{\prime}(\nu z \epsilon)-\alpha_{k} K_{\nu}(\nu z \epsilon)}\right) .
\end{aligned}
$$

For the arguments below we need to summarize some facts about Bessel functions. We consider expansions of Bessel-functions for large arguments and fixed order, (see $\left[\mathrm{ABST}_{9}\right.$ 2, p.377]). For the modified Bessel functions of first kind we have

$$
\begin{aligned}
& I_{\nu}(z)=\frac{e^{z}}{\sqrt{2 \pi z}}\left(1+O\left(\frac{1}{z}\right)\right), \\
& I_{\nu}^{\prime}(z)=\frac{e^{z}}{\sqrt{2 \pi z}}\left(1+O\left(\frac{1}{z}\right)\right),
\end{aligned}
$$


Expansions for modified Bessel functions of second kind are

$$
\begin{gathered}
K_{\nu}(z)=\sqrt{\frac{\pi}{2 z}} e^{-z}\left(1+O\left(\frac{1}{z}\right)\right), \\
K_{\nu}^{\prime}(z)=-\sqrt{\frac{\pi}{2 z}} e^{-z}\left(1+O\left(\frac{1}{z}\right)\right),
\end{gathered}
$$

The expansions (5.33) and (5.34) hold in $|\arg (z)|<\pi / 2$, in particular they hold for $z=\sqrt{-\lambda}$ with $\lambda \in \Lambda_{c}$ large. For small arguments and positive orders $\nu>0$ we have the following expansions

$$
\begin{gathered}
I_{\nu}(z) \sim \frac{z^{\nu}}{2^{\nu} \Gamma(\nu+1)}, \quad K_{\nu}(z) \sim 2^{\nu-1} \frac{\Gamma(\nu)}{z^{\nu}}, \quad \text { as }|z| \rightarrow 0 . \\
I_{\nu}^{\prime}(z) \sim \frac{z^{\nu-1}}{2^{\nu} \Gamma(\nu)}, \quad K_{\nu}^{\prime}(z) \sim-2^{\nu-1} \frac{\Gamma(\nu+1)}{z^{\nu+1}},
\end{gathered}
$$

Next recall the expansions of Bessel-functions for large order $\nu>0$ (see [OLV97, Section $7])$. For any $z \in\{w \in \mathbb{C}:|\arg (w)|<\pi / 2\} \cup\{i x \mid x \in(-1,1)\}$, put

$$
t:=\left(1+z^{2}\right)^{-1 / 2} \quad \text { and } \quad \xi:=1 / t+\log (z /(1+1 / t)) .
$$

For the modified Bessel functions of first kind we then have

$$
\begin{gathered}
I_{\nu}(\nu z)=\frac{1}{\sqrt{2 \pi \nu}} \frac{e^{\nu \xi}}{\left(1+z^{2}\right)^{1 / 4}}\left[1+\sum_{r=1}^{N-1} \frac{u_{r}(t)}{\nu^{r}}+\frac{\eta_{N, 1}(\nu, z)}{\nu^{N}}\right], \\
I_{\nu}^{\prime}(\nu z)=\frac{1}{\sqrt{2 \pi \nu}} \frac{e^{\nu \xi}}{z\left(1+z^{2}\right)^{-1 / 4}}\left[1+\sum_{r=1}^{N-1} \frac{v_{r}(t)}{\nu^{r}}+\frac{\eta_{N, 2}(\nu, z)}{\nu^{N}}\right] .
\end{gathered}
$$

Expansions for modified Bessel functions of second kind are

$$
\begin{array}{r}
K_{\nu}(\nu z)=\sqrt{\frac{\pi}{2 \nu}} \frac{e^{-\nu \xi}}{\left(1+z^{2}\right)^{1 / 4}}\left[1+\sum_{r=1}^{N-1} \frac{u_{r}(t)}{(-\nu)^{r}}+\frac{\eta_{N, 3}(\nu, z)}{(-\nu)^{N}}\right], \\
K_{\nu}^{\prime}(\nu z)=-\sqrt{\frac{\pi}{2 \nu}} \frac{e^{-\nu \xi}}{z\left(1+z^{2}\right)^{-1 / 4}}\left[1+\sum_{r=1}^{N-1} \frac{v_{r}(t)}{(-\nu)^{r}}+\frac{\eta_{N, 4}(\nu, z)}{(-\nu)^{N}}\right] .
\end{array}
$$

The error terms $\eta_{N, i}(\nu, z)$ are bounded for large $\nu$ uniformly in any compact subset of $\{z \in \mathbb{C}:|\arg (z)|<\pi / 2\} \cup\{i x \mid x \in(-1,1)\}$. For this fact see the analysis of the validity regions for the expansions (5.36) and (5.37) in [OLv97, Section 8]. For $\lambda \in \Lambda_{c}$ with $0<c<1$, the induced $z=\sqrt{-\lambda}$ is contained in that region of validity, where we use the main branch of logarithm in $\mathbb{C} \backslash \mathbb{R}^{-}$, extended by continuity to one of the sides of the cut. The coefficients $u_{r}(t), v_{r}(t)$ are polynomial in $t$ and defined via a recursive relation (see [OLv97, (7.10)]). 
As in [BKD96, (3.15)] we have for any fixed $\alpha \in \mathbb{R}$ the following expansion as $\nu \rightarrow \infty$

$$
\begin{aligned}
& \log \left(1+\sum_{r=1}^{N} \frac{u_{r}(t)}{( \pm \nu)^{r}}+O\left(\nu^{-N-1}\right)\right) \sim \sum_{r=1}^{\infty} \frac{D_{r}(t)}{( \pm \nu)^{r}}+O\left(\nu^{-N-1}\right), \\
& \log \left[\left(1+\sum_{k=1}^{N} \frac{v_{r}(t)}{( \pm \nu)^{r}}\right)+\frac{\alpha}{( \pm \nu)} t\left(1+\sum_{r=1}^{N-1} \frac{u_{r}(t)}{( \pm \nu)^{r}}\right)+O\left(\nu^{-N-1}\right)\right] \\
& \sim \sum_{r=1}^{N} \frac{M_{r}(t, \alpha)}{( \pm \nu)^{r}}+O\left(\nu^{-N-1}\right) .
\end{aligned}
$$

The coefficients $D_{r}(t)$ and $M_{r}(t, \alpha)$ are polynomial in $t$ of the form

$$
D_{r}(t)=\sum_{b=0}^{r} x_{r, b} t^{r+2 b}, \quad M_{r}(t, \alpha)=\sum_{b=0}^{r} z_{r, b}(\alpha) t^{r+2 b} .
$$

This follows from the fact that the $u_{r}(t)$ 's and $v_{r}(t)$ 's are polynomials. See also [BKD96, $(3.7),(3.16)]$. As a consequence of [BGKE96, (4.24)] we have

$$
M_{r}(1, \alpha)=D_{r}(1)-\frac{(-\alpha)^{r}}{r} \text {. }
$$

Proposition 5.7. There exist $\epsilon, c>0$ such that for $\operatorname{Re}(s) \gg 0$ we have

$$
\begin{aligned}
\zeta_{k}(s, \epsilon) & =\frac{s^{2}}{\Gamma(s+1)} \int_{0}^{\infty} \frac{t^{s-1}}{2 \pi i} \int_{\wedge_{c}} \frac{e^{-\lambda t}}{-\lambda} T_{\epsilon}^{k}(s, \lambda) d \lambda d t, \\
T_{\epsilon}^{k}(s, \lambda) & =\sum_{\eta \in E_{k}} \mathrm{~m}(\eta) t_{\eta, \epsilon}^{k}(\lambda) \nu(\eta)^{-2 s}
\end{aligned}
$$

Proof. Consider the expression of $t_{\eta, \epsilon}^{k}(\lambda)$ in (5.32) in terms of Bessel functions. We need to investigate its behavior for large $\eta$, or equivalently for large $\nu(\eta)$. Let $z \in\{w \in$ $\mathbb{C}:|\arg (w)|<\pi / 2\} \cup\{i x \mid x \in(-1,1)\}$ and $t_{\epsilon}:=\left(1+(\epsilon z)^{2}\right)^{-1 / 2}$. By (5.37) we find

$$
\begin{aligned}
-\log \left(\nu z \epsilon K_{\nu}^{\prime}(\nu z \epsilon)+\alpha_{k} K_{\nu}(\nu z \epsilon)\right)+\log \left(\nu z \epsilon K_{\nu}^{\prime}(\nu z \epsilon)-\alpha_{k} K_{\nu}(\nu z \epsilon)\right) \\
=-\log \left[\left(1+\sum_{k=1}^{N-1} \frac{v_{r}\left(t_{\epsilon}\right)}{(-\nu)^{r}}\right)+\frac{\alpha_{k}}{(-\nu)} t_{\epsilon}\left(1+\sum_{r=1}^{N-2} \frac{u_{r}\left(t_{\epsilon}\right)}{(-\nu)^{r}}\right)+\frac{\kappa_{N, 1}(\nu, z \epsilon)}{(-\nu)^{N}}\right] \\
+\log \left[\left(1+\sum_{k=1}^{N-1} \frac{v_{r}\left(t_{\epsilon}\right)}{(-\nu)^{r}}\right)-\frac{\alpha_{k}}{(-\nu)} t_{\epsilon}\left(1+\sum_{r=1}^{N-2} \frac{u_{r}\left(t_{\epsilon}\right)}{(-\nu)^{r}}\right)+\frac{\kappa_{N, 2}(\nu, z \epsilon)}{(-\nu)^{N}}\right],
\end{aligned}
$$

where the error terms

$$
\begin{aligned}
& \kappa_{N, 1}(\nu, z \epsilon)=\eta_{N, 4}(\nu, z \epsilon)+\left(\alpha_{k} t_{\epsilon}\right) \eta_{N-1,3}(\nu, z \epsilon) \\
& \kappa_{N, 2}(\nu, z \epsilon)=\eta_{N, 4}(\nu, z \epsilon)-\left(\alpha_{k} t_{\epsilon}\right) \eta_{N-1,3}(\nu, z \epsilon)
\end{aligned}
$$

are bounded for large $\nu$ uniformly in any compact subset of $\{z \in \mathbb{C}:|\arg (z)|<\pi / 2\} \cup$ $\{i x \mid x \in(-1,1)\}$. Employing (5.36) and (5.37) we find with $\xi:=1 / t+\log (z /(1+1 / t))$ 
and $\xi_{\epsilon}:=1 / t_{\epsilon}+\log \left(\epsilon z /\left(1+1 / t_{\epsilon}\right)\right)$

$$
\begin{aligned}
& \log \left(1-\frac{\nu z K_{\nu}^{\prime}(\nu z) \pm \alpha_{k} K_{\nu}(\nu z)}{\nu z I_{\nu}^{\prime}(\nu z) \pm \alpha_{k} I_{\nu}(\nu z)} \cdot \frac{\nu z \epsilon I_{\nu}^{\prime}(\nu z \epsilon) \pm \alpha_{k} I_{\nu}(\nu z \epsilon)}{\nu z \epsilon K_{\nu}^{\prime}(\nu z \epsilon) \pm \alpha_{k} K_{\nu}(\nu z \epsilon)}\right)= \\
& \log \left(1-e^{2 \nu\left(\xi_{\epsilon}-\xi\right)}(1+\kappa(\nu, z))\right)
\end{aligned}
$$

where the error term $\kappa(\nu, z)$ is again bounded for large $\nu$ uniformly in any compact subset of $\{z \in \mathbb{C}:|\arg (z)|<\pi / 2\} \cup\{i x \mid x \in(-1,1)\}$. We need to consider the difference $\left(\xi_{\epsilon}-\xi\right)$ in detail.

$$
\begin{aligned}
& \xi_{\epsilon}-\xi=\sqrt{1+(\epsilon z)^{2}}-\sqrt{1+z^{2}}+\log \left(\frac{\epsilon z}{1+\sqrt{1+(\epsilon z)^{2}}}\right)-\log \left(\frac{z}{1+\sqrt{1+z^{2}}}\right) \\
= & \sqrt{1+(\epsilon z)^{2}}\left[1-\frac{1}{\epsilon} \sqrt{\frac{\epsilon^{2}+(\epsilon z)^{2}}{1+(\epsilon z)^{2}}}\right]+\log \left(\frac{\epsilon z}{1+\sqrt{1+(\epsilon z)^{2}}}\right)-\log \left(\frac{z}{1+\sqrt{1+z^{2}}}\right) .
\end{aligned}
$$

We are interested in the asymptotic behavior of $\left(\xi_{\epsilon}-\xi\right)$ as $\epsilon \rightarrow 0$, which is possibly non-uniform in $z$. Hence, we consider $\left(\xi_{\epsilon}-\xi\right)$ under three asymptotic regimes, $|\epsilon z| \rightarrow$ $\infty,|\epsilon z| \rightarrow 0$ and $|\epsilon z| \sim$ const. We find by straightforward estimates

$$
\begin{aligned}
& \operatorname{Re}\left(\xi_{\epsilon}-\xi\right) \sim \epsilon \operatorname{Re}(z)(1-1 / \epsilon)=\operatorname{Re}(z)(\epsilon-1), \text { as }|\epsilon z| \rightarrow \infty, \epsilon \rightarrow 0, \\
& \operatorname{Re}\left(\xi_{\epsilon}-\xi\right) \sim \log |\epsilon z|-\operatorname{Re} \sqrt{1+z^{2}}, \text { as }|\epsilon z| \rightarrow 0, \epsilon \rightarrow 0, \\
& \operatorname{Re}\left(\xi_{\epsilon}-\xi\right) \sim-C \epsilon^{-1}, \text { as }|\epsilon z| \sim \text { const, } \epsilon \rightarrow 0,
\end{aligned}
$$

for some constant $C>0$. For $\{z \in \mathbb{C}:|\arg (z)|<\pi / 2\} \cup\{z=i x \mid x \in(-1,1)\}$, we have $\operatorname{Re} \sqrt{1+z^{2}}>0$, and $\operatorname{Re}(z)>0$ as $|z| \rightarrow \infty$. Consequently, for $\epsilon>0$ sufficiently small $\operatorname{Re}\left(\xi_{\epsilon}-\xi\right)<\delta<0$ for some fixed $\delta<0$ and hence $\exp \left(2 \nu\left(\xi_{\epsilon}-\xi\right)\right)$ vanishes as $\nu \rightarrow \infty$, uniformly in any compact subset of $\{z \in \mathbb{C}:|\arg (z)|<\pi / 2\} \cup\{i x \mid x \in(-1,1)\}$.

The uniform expansions above show that in (5.41) and (5.43) the arguments of the logarithms stay away from the branch cut $\mathbb{C} \backslash \mathbb{R}^{-}$for $\nu$ large enough and $\epsilon>0$ sufficiently small, uniformly in any compact subset of $\{z \in \mathbb{C}:|\arg (z)|<\pi / 2\} \cup\{i x \mid x \in(-1,1)\}$. Consequently, in view of the expression (5.32), $t_{\eta, \epsilon}^{k}(\lambda)$ is in particular holomorphic in an open neighborhood of $\left\{\lambda \in\left[0, c^{\prime}\right]\right\} \subset \mathbb{C}$ for some $0<c^{\prime}<1$ and $\nu(\eta)>\nu_{0}$. Moreover, for any $\eta \in E_{k}, t_{\eta, \epsilon}^{k}(\lambda)$ is holomorphic in $\lambda \in \mathbb{C} \backslash\{x \in \mathbb{R} \mid x>c(\eta)\}$. Thus, setting $c:=\min \left\{c^{\prime}, c(\eta) \mid \eta \in E_{k}, \nu(\eta) \leq \nu_{0}\right\}$, we deduce for $\epsilon>0$ sufficiently small

$$
\zeta_{k}(s, \epsilon)=\sum_{\eta \in E_{k}} \mathrm{~m}(\eta) \nu(\eta)^{-2 s} \frac{s^{2}}{\Gamma(s+1)} \int_{0}^{\infty} \frac{t^{s-1}}{2 \pi i} \int_{\wedge_{c}} \frac{e^{-\lambda t}}{-\lambda} t_{\eta, \epsilon}^{k}(\lambda) d \lambda d t,
$$

The deforming of the contour of integration from $\Lambda_{c(\eta)}$ to $\Lambda_{c}$ is permissible, as the deformation is performed within the region of regularity for each $t_{\eta, \epsilon}^{k}(\lambda), \eta \in E_{k}$. Employing again the expansions (5.36) and (5.37) we find that

$$
\sum_{\eta \in E_{k}} \mathrm{~m}(\eta) t_{\eta, \epsilon}^{k}(\lambda) \nu(\eta)^{-2 s}, \operatorname{Re}(s) \gg 0,
$$

converges uniformly in $\lambda \in \Lambda_{c}$ and hence by the uniform convergence of the integrals and series we arrive at the statement of the proposition. 
Proposition 5.8. Let the notation be as in Proposition 5.5 and 5.7. Let $\lambda \in \Lambda_{c}$ and $t_{\epsilon}(\lambda):=\left(1-\left(\epsilon^{2} \lambda\right)\right)^{-1 / 2}$. Then for $\epsilon>0$ sufficiently small we have the following asymptotic expansion for large $\nu(\eta), \eta \in E_{k}$

$$
t_{\eta, \epsilon}^{k}(\lambda) \sim \sum_{r=1}^{\infty} \frac{1}{(-\nu(\eta))^{r}}\left(M_{r}\left(t_{\epsilon}(\lambda),-\alpha_{k}\right)-M_{r}\left(t_{\epsilon}(\lambda), \alpha_{k}\right)+\left(\frac{\alpha_{k}^{r}-\left(-\alpha_{k}\right)^{r}}{r}\right)\right) .
$$

Proof. Consider expansions of the individual terms in the expression (5.32) for $t_{\eta, \epsilon}^{k}(\lambda)$. For $\lambda \in \Lambda_{c}, z=\sqrt{-\lambda}$ lies in the region of validity of the expansions (5.36) and (5.37). Combining (5.37) and (5.38) we compute for large $\nu \equiv \nu(\eta)$

$$
\begin{array}{r}
-\log \left(\nu z \epsilon K_{\nu}^{\prime}(\nu z \epsilon)+\alpha_{k} K_{\nu}(\nu z \epsilon)\right)+\log \left(\nu z \epsilon K_{\nu}^{\prime}(\nu z \epsilon)-\alpha_{k} K_{\nu}(\nu z \epsilon)\right) \\
\sim-\log \left[\left(1+\sum_{k=1}^{\infty} \frac{v_{r}\left(t_{\epsilon}(\lambda)\right)}{(-\nu)^{r}}\right)+\frac{\alpha_{k}}{(-\nu)} t_{\epsilon}(\lambda)\left(1+\sum_{r=1}^{\infty} \frac{u_{r}\left(t_{\epsilon}(\lambda)\right)}{\left.(-\nu)^{r}\right)}\right]\right. \\
+\log \left[\left(1+\sum_{k=1}^{\infty} \frac{v_{r}\left(t_{\epsilon}(\lambda)\right)}{(-\nu)^{r}}\right)-\frac{\alpha_{k}}{(-\nu)} t_{\epsilon}(\lambda)\left(1+\sum_{r=1}^{\infty} \frac{u_{r}\left(t_{\epsilon}(\lambda)\right)}{(-\nu)^{r}}\right)\right] \\
\sim \sum_{r=1}^{\infty} \frac{1}{(-\nu)^{r}}\left(-M_{r}\left(t_{\epsilon}(\lambda), \alpha_{k}\right)+M_{r}\left(t_{\epsilon}(\lambda),-\alpha_{k}\right)\right), \text { as } \nu \rightarrow \infty .
\end{array}
$$

The standard expansion of the logarithm yields for large $\nu$

$$
-\log \left(1+\frac{\alpha_{k}}{\nu}\right)+\log \left(1-\frac{\alpha_{k}}{\nu}\right)=\sum_{r=1}^{\infty} \frac{1}{(-\nu)^{r}}\left(\frac{\alpha_{k}^{r}-\left(-\alpha_{k}\right)^{r}}{r}\right) .
$$

This already gives all the terms in the stated asymptotic expansion of $t_{\eta, \epsilon}^{k}\left(-z^{2}\right)$. Thus we need to check that the remaining terms indeed have no asymptotic contribution. Using (5.36), (5.37), and putting $\xi_{\epsilon}:=1 / t_{\epsilon}(\lambda)+\log \left(\epsilon z /\left(1+1 / t_{\epsilon}(\lambda)\right)\right)$, the remaining terms are estimated as follows

$$
\frac{\nu z K_{\nu}^{\prime}(\nu z) \pm \alpha_{k} K_{\nu}(\nu z)}{\nu z I_{\nu}^{\prime}(\nu z) \pm \alpha_{k} I_{\nu}(\nu z)} \cdot \frac{\nu z \epsilon I_{\nu}^{\prime}(\nu z \epsilon) \pm \alpha_{k} I_{\nu}(\nu z \epsilon)}{\nu z \epsilon K_{\nu}^{\prime}(\nu z \epsilon) \pm \alpha_{k} K_{\nu}(\nu z \epsilon)} \sim O\left(e^{2 \nu\left(\xi_{\epsilon}-\xi\right)}\right), \nu \rightarrow \infty
$$

The difference $\left(\xi_{\epsilon}-\xi\right)$ has been considered in detail in Proposition 5.7. For $\epsilon$ sufficiently small, $\operatorname{Re}\left(\xi_{\epsilon}-\xi\right)<0$ and hence the remainder term $O\left(e^{2 \nu\left(\xi_{\epsilon}-\xi\right)}\right)$ in (5.46) does not contribute to the asymptotic expansion for large $\nu$.

Next we introduce a (shifted) zeta-function by

$$
\zeta_{k, N}(s):=\sum_{\eta \in E_{k}} \mathrm{~m}(\eta) \nu(\eta)^{-s}=\zeta\left(\frac{s}{2}, \Delta_{k, c c l, N}+\alpha_{k}^{2}\right), \operatorname{Re}(s)>n,
$$

where as before $\mathrm{m}(\eta)$ denotes the multiplicity of $\eta \in E_{k}$. The heat trace expansions for $\left(\Delta_{k, c c l, N}+\alpha_{k}^{2}\right)$ and $\Delta_{k, c c l, N}$ have the same exponents, and hence $\zeta_{k, N}(s)$ extends meromorphically to $\mathbb{C}$ with simple poles at $\{(n-2 k) \mid k \in \mathbb{N}\}$. Consequently, the terms $\nu(\eta)^{-r}$ in the asymptotic expansion of $t_{\eta, \epsilon}^{k}(\lambda)$ with $r=n-2 k, k \in \mathbb{N}$, may lead to 
singular behavior of $T_{\epsilon}^{k}(s, \lambda)$ at $s=0$. We regularize $T_{\epsilon}^{k}(s, \lambda)$ by subtracting off these terms from $t_{\eta, \epsilon}^{k}(\lambda)$, and define

$$
\begin{aligned}
& f_{r, \epsilon}^{k}(\lambda):=(-1)^{r}\left(M_{r}\left(t_{\epsilon}(\lambda),-\alpha_{k}\right)-M_{r}\left(t_{\epsilon}(\lambda), \alpha_{k}\right)+\left(\frac{\alpha_{k}^{r}-\left(-\alpha_{k}\right)^{r}}{r}\right)\right), \\
& p_{\eta, \epsilon}^{k}(\lambda):=t_{\eta, \epsilon}^{k}(\lambda)-\sum_{r=1}^{n} \nu(\eta)^{-r} f_{r, \epsilon}^{k}(\lambda), \quad P_{\epsilon}^{k}(s, \lambda):=\sum_{\eta \in E_{k}} \mathrm{~m}(\eta) p_{\eta, \epsilon}^{k}(\lambda) \nu(\eta)^{-2 s} .
\end{aligned}
$$

By construction, $P_{\epsilon}^{k}(s, \lambda)$ is regular at $s=0$. The contribution of the terms $f_{r, \epsilon}^{k}(\lambda)$ is computed in terms of the polynomials $M_{r}(t, \alpha)$ in (5.39). The computation uses special integrals evaluated already by Spreafico [SPR06].

\section{Proposition 5.9.}

$$
\int_{0}^{\infty} t^{s-1} \frac{1}{2 \pi i} \int_{\Lambda_{c}} \frac{e^{-\lambda t}}{-\lambda} f_{r, \epsilon}^{k}(\lambda) d \lambda d t=(-1)^{r} \sum_{b=0}^{r}\left(z_{r, b}\left(-\alpha_{k}\right)-z_{r, b}\left(\alpha_{k}\right)\right) \frac{\Gamma(s+b+r / 2)}{s \Gamma(b+r / 2)} \epsilon^{2 s} .
$$

Proof. The $\lambda$-independent part of $f_{r, \epsilon}^{k}(\lambda)$ vanishes after integration in $\lambda$. The coefficients $M_{r}\left(t_{\epsilon}(\lambda), \pm \alpha_{k}\right)$ in the definition of $f_{r, \epsilon}^{k}(\lambda)$ are polynomial in $t_{\epsilon}(\lambda)=\left(1-\epsilon^{2} \lambda\right)^{-1 / 2}$. Hence we compute, by substituting first $\mu=\epsilon^{2} \lambda$, and then $\tau=t / \epsilon^{2}$

$$
\int_{0}^{\infty} t^{s-1} \frac{1}{2 \pi i} \int_{\wedge_{c}} \frac{e^{-\lambda t}}{-\lambda} \frac{1}{\left(1-\epsilon^{2} \lambda\right)^{a}} d \lambda d t=\epsilon^{2 s} \int_{0}^{\infty} \tau^{s-1} \frac{1}{2 \pi i} \int_{\wedge_{\epsilon^{2} c}} \frac{e^{-\mu \tau}}{-\mu} \frac{1}{(1-\mu)^{a}} d \mu d \tau
$$

For the inner integral we obtain by substituting $z=\tau(\mu-1)$

$$
\frac{1}{2 \pi i} \int_{\wedge_{\epsilon^{2} c}} \frac{e^{-\mu \tau}}{-\mu} \frac{1}{(1-\mu)^{a}} d \mu=-\frac{1}{2 \pi i} e^{-\tau} \tau^{a} \int_{\wedge} \frac{e^{-z}}{z+\tau}(-z)^{-a} d z,
$$

where $\wedge \equiv \wedge_{\tau\left(\epsilon^{2} c-1\right)}$. The contour of integration encircles a pole singularity $z=0$ of the integrand and the second pole at $z=-\tau$ lies outside the contour of integration. Hence we can deform the contour to start at infinity of the real axis, continue along real axis to some $\delta>0$, continue along the circle of radius $\delta$ around the origin counter-clockwise, and then continue from $\delta$ back to infinity along the real axis.

The deformation does not change the value of the integral, and the deformed contour shall be denoted by $\mathscr{C}_{\delta}$, with its three components $\mathscr{C}_{\delta}^{j}, j=1,2,3$, as in Figure 5.3 below. We can now evaluate the integral along each of these three components.

Note that the many-valued function $(-z)^{-a}$ is made definite by the convention

$$
(-z)^{-a}=e^{-a \log (-z)},
$$

with the main branch of the logarithm. Along $\mathscr{C}_{\delta}^{1}$ we have $\arg (-z)=-\pi$, and along $\mathscr{C}_{\delta}^{3}$ we have $\arg (-z)=\pi$. Consequently, we find

$$
-\frac{1}{2 \pi i} e^{-\tau} \tau^{a} \int_{\mathscr{C}_{\delta}^{1} \cup \mathscr{C}_{\delta}^{2}} \frac{e^{-z}}{z+\tau}(-z)^{-a} d z=\frac{\sin (a \pi)}{\pi} e^{-\tau} \tau^{a} \int_{\delta}^{\infty} \frac{e^{-z}}{z+\tau} z^{-a} d z
$$




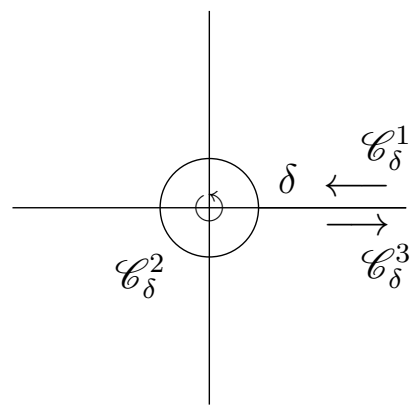

Figure 3. The deformed integration contour $\mathscr{C}_{\delta}$.

Assuming $\operatorname{Re}(a)<1$, the limits as $\delta \rightarrow 0$ for the integrals along each of the three components $\mathscr{C}_{\delta}^{j}, j=1,2,3$, are well defined and in fact the integral along $\mathscr{C}_{\delta}^{2}$ vanishes in the limit. Consequently we obtain using [GRRY94, 8.353.3] and assuming $\operatorname{Re}(a)<1$

$$
\begin{aligned}
-\frac{1}{2 \pi i} e^{-\tau} \tau^{a} \int_{\wedge} \frac{e^{-z}}{z+\tau}(-z)^{-a} d z & =\frac{\sin (a \pi)}{\pi} e^{-\tau} \tau^{a} \int_{0}^{\infty} \frac{e^{-z}}{z+\tau} z^{-a} d z \\
& =\frac{\sin (a \pi)}{\pi} \Gamma(a, \tau) \Gamma(1-a) .
\end{aligned}
$$

Since the left integral in (5.49) and the expression (5.50) are both analytic in $a \in \mathbb{C}$, the equality between the two in fact holds for any $a \in \mathbb{C}$ and the statement follows finally from the relation between the incomplete Gamma function and the probability integral

$$
\int_{0}^{\infty} \tau^{s-1} \frac{\Gamma(a, \tau)}{\Gamma(a)} d \tau=\frac{\Gamma(s+a)}{s \Gamma(a)} .
$$

Consequently we arrive at the intermediate representation of $\zeta_{k}(s, \epsilon)$ for $\operatorname{Re}(s) \gg 0$

$$
\begin{aligned}
\zeta_{k}(s, \epsilon) & =\frac{s^{2}}{\Gamma(s+1)} \int_{0}^{\infty} \frac{t^{s-1}}{2 \pi i} \int_{\wedge_{c}} \frac{e^{-\lambda t}}{-\lambda} P_{\epsilon}^{k}(s, \lambda) d \lambda d t \\
& +\sum_{r=1}^{n} \zeta_{k, N}(2 s+r) \frac{(-1)^{r} s}{\Gamma(s+1)} \sum_{b=0}^{r}\left(z_{r, b}\left(-\alpha_{k}\right)-z_{r, b}\left(\alpha_{k}\right)\right) \frac{\Gamma(s+b+r / 2)}{\Gamma(b+r / 2)} \epsilon^{2 s}
\end{aligned}
$$

While the second summand in (5.52) extends meromorphically to $\mathbb{C}$, it still remains to derive an analytic extension to $s=0$ for the first summand.

Proposition 5.10. Let the notation be as in Proposition 5.5 and (5.48). Then for large arguments $\lambda \in \Lambda_{c}$ and fixed order $\eta \in E_{k}$ we have the following asymptotics

$$
p_{\eta, \epsilon}^{k}(\lambda)=b_{\eta, \epsilon}^{k}+O\left((-\lambda)^{-1 / 2}\right),
$$

where

$$
b_{\eta, \epsilon}^{k}=\log \left(1-\frac{\alpha_{k}}{\nu(\eta)}\right)-\log \left(1+\frac{\alpha_{k}}{\nu(\eta)}\right)-\sum_{r=1}^{n} \frac{1}{(-\nu(\eta))^{r}}\left(\frac{\alpha_{k}^{r}-\left(-\alpha_{k}\right)^{r}}{r}\right) .
$$


Proof. The function $p_{\eta, \epsilon}^{k}(\lambda)$ is given by the following expression

$$
p_{\eta, \epsilon}^{k}(\lambda)=t_{\eta, \epsilon}^{k}(\lambda)-\sum_{r=1}^{n} \frac{1}{(-\nu(\eta))^{r}}\left(M_{r}\left(t_{\epsilon}(\lambda),-\alpha_{k}\right)-M_{r}\left(t_{\epsilon}(\lambda), \alpha_{k}\right)+\left(\frac{\alpha_{k}^{r}-\left(-\alpha_{k}\right)^{r}}{r}\right)\right) .
$$

The polynomials $M_{2 r}\left(t_{\epsilon}(\lambda), \pm \alpha_{k}\right)$ have no constant terms, and hence $M_{2 r}\left(t_{\epsilon}(\lambda), \pm \alpha_{k}\right) \sim$ $O\left((-\lambda)^{-1 / 2}\right), \lambda \rightarrow \infty$, since

$$
t_{\epsilon}(\lambda)=\frac{1}{\sqrt{1-\epsilon^{2} \lambda}}=O\left((-\lambda)^{-1 / 2}\right), \quad \lambda \rightarrow \infty .
$$

By (5.33) and (5.34), setting $\nu \equiv \nu(\eta)$

$$
\begin{aligned}
& -\log \left(\nu z \epsilon K_{\nu}^{\prime}(\nu z \epsilon)+\alpha_{k} K_{\nu}(\nu z \epsilon)\right)+\log \left(\nu z \epsilon K_{\nu}^{\prime}(\nu z \epsilon)-\alpha_{k} K_{\nu}(\nu z \epsilon)\right) \\
& \sim \log \left(1+\frac{\alpha_{k}}{\nu z \epsilon}\right)-\log \left(1-\frac{\alpha_{k}}{\nu z \epsilon}\right)+O\left((-\lambda)^{-1 / 2}\right) \sim O\left((-\lambda)^{-1 / 2}\right), \quad \lambda \rightarrow \infty
\end{aligned}
$$

Moreover

$$
\frac{\nu z K_{\nu}^{\prime}(\nu z) \pm \alpha_{k} K_{\nu}(\nu z)}{\nu z I_{\nu}^{\prime}(\nu z) \pm \alpha_{k} I_{\nu}(\nu z)} \cdot \frac{\nu z \epsilon I_{\nu}^{\prime}(\nu z \epsilon) \pm \alpha_{k} I_{\nu}(\nu z \epsilon)}{\nu z \epsilon K_{\nu}^{\prime}(\nu z \epsilon) \pm \alpha_{k} K_{\nu}(\nu z \epsilon)} \sim O\left(e^{2 \nu z(\epsilon-1)}\right), \lambda \rightarrow \infty
$$

$(\epsilon-1)<0$ and $\operatorname{Re}(z)>0$ for large $z=\sqrt{-\lambda}, \lambda \in \Lambda_{c}$. Consequently $O\left(e^{2 \nu z(\epsilon-1)}\right)$ is in particular of $O\left((-\lambda)^{-1 / 2}\right)$ asymptotics for $\lambda \rightarrow \infty, \lambda \in \Lambda_{c}$. By the explicit expression for $t_{\eta, \epsilon}^{k}(\lambda)$ in (5.32) the statement follows.

Definition 5.11. Define for $\operatorname{Re}(s)>n$ in notation of Proposition 5.10

$$
B_{\epsilon}^{k}(s):=\sum_{\eta \in E_{k}} \mathrm{~m}(\eta) b_{\eta, \epsilon}^{k} \nu(\eta)^{-2 s} .
$$

$B_{\epsilon}^{k}(s)$ converges at $s=0$ by construction, since $\zeta_{k, N}(s)$ converges for $\operatorname{Re}(s)>n$.

Proposition 5.12. Let $P_{\epsilon}^{k}(s, \lambda)$ be defined by (5.48). Then

$$
P_{\epsilon}^{k}(s, 0)=0 \text {. }
$$

Proof. By (5.40)

$$
M_{r}\left(1,-\alpha_{k}\right)-M_{r}\left(1, \alpha_{k}\right)=\frac{\left(-\alpha_{k}\right)^{r}-\alpha_{k}^{r}}{r}
$$

For any fixed $\epsilon>0$ clearly $\lambda \rightarrow 0$ implies that $t=\left(1-\epsilon^{2} \lambda\right)^{-1 / 2}$ tends to 1 . Hence

$$
f_{r, \epsilon}^{k}(\lambda)=(-1)^{r}\left(M_{r}\left(1,-\alpha_{k}\right)-M_{r}\left(1, \alpha_{k}\right)+\left(\frac{\alpha_{k}^{r}-\left(-\alpha_{k}\right)^{r}}{r}\right)\right)=0 .
$$

Moreover, by (5.35)

$$
\begin{array}{r}
-\log \left(\nu z \epsilon K_{\nu}^{\prime}(\nu z \epsilon)+\alpha_{k} K_{\nu}(\nu z \epsilon)\right)+\log \left(\nu z \epsilon K_{\nu}^{\prime}(\nu z \epsilon)-\alpha_{k} K_{\nu}(\nu z \epsilon)\right) \\
\sim \log \left(1+\frac{\alpha_{k}}{\nu}\right)-\log \left(1-\frac{\alpha_{k}}{\nu}\right), \text { as } \lambda \rightarrow 0 .
\end{array}
$$

Moreover we have

$$
\frac{\nu z K_{\nu}^{\prime}(\nu z) \pm \alpha_{k} K_{\nu}(\nu z)}{\nu z I_{\nu}^{\prime}(\nu z) \pm \alpha_{k} I_{\nu}(\nu z)} \cdot \frac{\nu z \epsilon I_{\nu}^{\prime}(\nu z \epsilon) \pm \alpha_{k} I_{\nu}(\nu z \epsilon)}{\nu z \epsilon K_{\nu}^{\prime}(\nu z \epsilon) \pm \alpha_{k} K_{\nu}(\nu z \epsilon)} \sim \epsilon^{2 \nu}, \text { as } \lambda \rightarrow 0
$$


By the explicit expression for $t_{\eta, \epsilon}^{k}(\lambda)$ in (5.32) the statement follows. Note that the $\epsilon$-dependence cancels.

We can now put everything together and write down the meromorphic continuation to $s=0$ of the zeta-function $\zeta_{k}(s, \epsilon)$, introduced in Proposition 5.5. By the arguments of [Spr05, Section 4.1] we have

$$
\begin{aligned}
\zeta_{k}(s, \epsilon) & =\frac{s}{\Gamma(s+1)}\left(P_{\epsilon}^{k}(s, 0)-B_{\epsilon}^{k}(s)\right) \\
& +\sum_{r=1}^{n} \zeta_{k, N}(2 s+r) \frac{(-1)^{r} s}{\Gamma(s+1)} \sum_{b=0}^{r}\left(z_{r, b}\left(-\alpha_{k}\right)-z_{r, b}\left(\alpha_{k}\right)\right) \frac{\Gamma(s+b+r / 2)}{\Gamma(b+r / 2)} \epsilon^{2 s} \\
& +\frac{s^{2}}{\Gamma(s+1)} h(s, \epsilon),
\end{aligned}
$$

where $h(s, \epsilon)$ vanishes with its derivative at $s=0$. Note that all the terms are regular at $s=0$. Inserting the results of Proposition 5.9, Proposition 5.10, Proposition 5.12 together with Definition 5.11 into the expression (5.56) we obtain the following

Proposition 5.13. Let $\left(E_{N}, \nabla_{N}, h_{N}\right)$ be a flat Hermitian vector bundle over an evendimensional oriented closed Riemannian manifold $\left(N^{n}, g^{N}\right)$. Denote by $\Delta_{k, c c l, N}$ the Laplacian on coclosed $k$-differential forms $\Omega_{\mathrm{ccl}}^{k}\left(N, E_{N}\right)$. Let the notation be as in (5.39) and (5.47). Put

$$
\alpha_{k}:=\frac{(n-1)}{2}-k, \quad \nu(\eta)=\sqrt{\eta+\alpha_{k}^{2}}, \text { for } \eta \in E_{k}=\operatorname{Spec} \Delta_{k, c c l, N} \backslash\{0\} .
$$

Let $\mathrm{m}(\eta)$ denote the multiplicity of $\eta \in E_{k}$. Then for $\epsilon>0$ sufficiently small, $\zeta_{k}(s, \epsilon)$ defined in Definition 5.4 admits an analytic continuation to $s=0$ of the form

$$
\begin{aligned}
\zeta_{k}(s, \epsilon) & =\frac{s}{\Gamma(s+1)}\left(\sum_{\eta \in E_{k}} \mathrm{~m}(\eta) \nu(\eta)^{-2 s}\left(\log \left(1+\frac{\alpha_{k}}{\nu(\eta)}\right)+\sum_{r=1}^{n} \frac{\left(-\alpha_{k}\right)^{r}}{r \nu(\eta)^{r}}\right)\right) \\
& -\frac{s}{\Gamma(s+1)}\left(\sum_{\eta \in E_{k}} \mathrm{~m}(\eta) \nu(\eta)^{-2 s}\left(\log \left(1-\frac{\alpha_{k}}{\nu(\eta)}\right)+\sum_{r=1}^{n} \frac{\alpha_{k}^{r}}{r \nu(\eta)^{r}}\right)\right) \\
& +\sum_{r=1}^{n} \zeta_{k, N}(2 s+r) \frac{(-1)^{r} s}{\Gamma(s+1)} \sum_{b=0}^{r}\left(z_{r, b}\left(-\alpha_{k}\right)-z_{r, b}\left(\alpha_{k}\right)\right) \\
& \times \frac{\Gamma(s+b+r / 2)}{\Gamma(b+r / 2)} \epsilon^{2 s}+\frac{s^{2}}{\Gamma(s+1)} h(s),
\end{aligned}
$$

where $h(s)$ vanishes with its derivative at $s=0$.

Note the full analogy (up to computationally irrelevant, but geometrically crucial sign differences) to the corresponding result in [VER09A, Proposition 6.10]. An ad verbatim repetition of the arguments in the proof of [VER09A, Corollary 6.1] leads to the final formula. 
Corollary 5.14. Let $\left(E_{N}, \nabla_{N}, h_{N}\right)$ be a flat Hermitian vector bundle over an evendimensional oriented closed Riemannian manifold $\left(N^{n}, g^{N}\right)$. Denote by $\Delta_{k, c c l, N}$ the Laplacian on coclosed $k$-differential forms $\Omega_{\mathrm{ccl}}^{k}\left(N, E_{N}\right)$ and put

$$
\begin{aligned}
& \alpha_{k}:=\frac{(n-1)}{2}-k, \quad \nu(\eta)=\sqrt{\eta+\alpha_{k}^{2}}, \text { for } \eta \in E_{k}=\operatorname{Spec} \Delta_{k, c c l, N} \backslash\{0\}, \\
& \zeta_{k, N}(s)=\sum_{\eta \in E_{k}} \mathrm{~m}(\eta) \nu(\eta)^{-s}, \quad \zeta_{k, N}\left(s, \pm \alpha_{k}\right):=\sum_{\eta \in E_{k}} \mathrm{~m}(\eta)\left(\nu(\eta) \pm \alpha_{k}\right)^{-s}, \quad \operatorname{Re}(s) \gg 0
\end{aligned}
$$

where $\mathrm{m}(\eta)$ denotes the multiplicity of $\eta \in E_{k}$. Then we find in notation of (5.39) for $\epsilon>0$ sufficiently small

$$
\begin{aligned}
\zeta_{k}^{\prime}(0, \epsilon) & =\frac{1}{2} \sum_{r=1}^{n / 2} \operatorname{Res}_{s=2 r} \zeta_{k, N}(s) \sum_{b=0}^{2 r}\left(z_{2 r, b}\left(-\alpha_{k}\right)-z_{2 r, b}\left(\alpha_{k}\right)\right) \frac{\Gamma^{\prime}(b+r)}{\Gamma(b+r)} \\
& +\zeta_{k, N}^{\prime}\left(0,-\alpha_{k}\right)-\zeta_{k, N}^{\prime}\left(0, \alpha_{k}\right) .
\end{aligned}
$$

Proof. We follow the approach of [BKD96, Section 11]. Define

$$
K\left(s, \pm \alpha_{k}\right):=\sum_{\eta \in E_{k}} \mathrm{~m}(\eta) \nu(\eta)^{-2 s}\left(-\log \left(1 \pm \frac{\alpha_{k}}{\nu(\eta)}\right)-\sum_{r=1}^{n} \frac{\left(\mp \alpha_{k}\right)^{r}}{r \nu(\eta)^{r}}\right) .
$$

The series $K\left(0, \pm \alpha_{k}\right)$ converges absolutely, since $\zeta_{k, N}(s)$ converges absolutely for $R e(s) \geq n$. In order to evaluate $K\left(0, \pm \alpha_{k}\right)$, define

$$
\begin{aligned}
K_{0}\left(s, \pm \alpha_{k}\right) & :=\sum_{\eta \in E_{k}} \mathrm{~m}(\eta) \int_{0}^{\infty} t^{s-1} e^{-\nu(\eta) t}\left(e^{\mp \alpha_{k} t}-\sum_{r=0}^{n} \frac{\left(\mp \alpha_{k} t\right)^{r}}{r !}\right) d t \\
& =\Gamma(s) \zeta_{k, N}\left(s, \pm \alpha_{k}\right)-\sum_{r=0}^{n} \frac{\left(\mp \alpha_{k}\right)^{r}}{r !} \Gamma(s+r) \zeta_{k, N}(s+r) .
\end{aligned}
$$

$K_{0}\left(s, \pm \alpha_{k}\right)$ is an absolutely convergent sum at $s=0$, since $\zeta_{k, N}(s)$ converges absolutely for $\operatorname{Re}(s) \geq n$. By construction

$$
K\left(0, \pm \alpha_{k}\right)=K_{0}\left(0, \pm \alpha_{k}\right) .
$$

Furthermore we find from (5.58) and regularity of $K_{0}\left(s, \pm \alpha_{k}\right)$ at $s=0$

$$
\zeta_{k, N}\left(0, \pm \alpha_{k}\right)=\zeta_{k, N}(0)+\sum_{r=1}^{n} \frac{\left(\mp \alpha_{k}\right)^{r}}{r} \operatorname{Res}_{s=r} \zeta_{k, N}(s)
$$

Using (5.60) we obtain following expansion at $s=0$

$$
\begin{aligned}
\Gamma(s) \zeta_{k, N}\left(s, \pm \alpha_{k}\right) & \sim\left(\frac{1}{s}-\gamma+O(s)\right)\left(\zeta_{k, N}\left(s, \pm \alpha_{k}\right)-\zeta_{k, N}\left(0, \pm \alpha_{k}\right)\right) \\
& +\left(\frac{1}{s}-\gamma+O(s)\right) \zeta_{k, N}\left(0, \pm \alpha_{k}\right) \sim \zeta_{k, N}^{\prime}\left(0, \pm \alpha_{k}\right) \\
& +\left(\frac{1}{s}-\gamma\right)\left(\zeta_{k, N}(0)+\sum_{r=1}^{n} \frac{\left(\mp \alpha_{k}\right)^{r}}{r} \operatorname{Res}_{s=r} \zeta_{k, N}(s)\right)+O(s) .
\end{aligned}
$$


Similarly we find

$$
\Gamma(s) \zeta_{k, N}(s) \sim \zeta_{k, N}^{\prime}(0)+\left(\frac{1}{s}-\gamma\right) \zeta_{k, N}(0)+O(s), \text { as } s \rightarrow 0 .
$$

Moreover, denoting by $\mathrm{PP} \zeta_{k, N}(r)$ the constant term in the asymptotics of $\zeta_{k, N}(s)$ near the pole singularity $s=r$, we compute

$$
\begin{aligned}
\sum_{r=1}^{n} \frac{\left(\mp \alpha_{k}\right)^{r}}{r !} \Gamma(s+r) \zeta_{k, N}(s+r) & \sim \sum_{r=1}^{n} \frac{\left(\mp \alpha_{k}\right)^{r}}{r !} \frac{\Gamma(s+r)}{s} \operatorname{Res}_{s=r} \zeta_{k, N}(s) \\
& +\sum_{r=1}^{n} \frac{\left(\mp \alpha_{k}\right)^{r}}{r} \operatorname{PP} \zeta_{k, N}(r)+O(s), \text { as } s \rightarrow 0 .
\end{aligned}
$$

Plugging (5.61), (5.62) and (5.63) into (5.58) we arrive at the following

$$
\begin{aligned}
K\left(0, \pm \alpha_{k}\right) & =K_{0}\left(0, \pm \alpha_{k}\right)=\zeta_{k, N}^{\prime}(0, \alpha)-\zeta_{k, N}^{\prime}(0) \\
& -\sum_{r=1}^{n} \frac{\left(\mp \alpha_{k}\right)^{r}}{r}\left(\operatorname{Res}_{s=r} \zeta_{k, N}(s)\left(\gamma+\frac{\Gamma^{\prime}(r)}{\Gamma(r)}\right)+\operatorname{PP} \zeta_{k, N}(r)\right),
\end{aligned}
$$

This result corresponds to the result obtained in [BKD96, p. 388], up to certain factors due to a different notation. Furthermore, we compute straightforwardly

$$
\left.\frac{d}{d s}\right|_{0} \zeta_{k, N}(2 s+r) \frac{s}{\Gamma(s+1)} \frac{\Gamma\left(s+b+\frac{r}{2}\right)}{\Gamma\left(b+\frac{r}{2}\right)}=\frac{1}{2} \underset{s=r}{\operatorname{Res} \zeta_{k, N}(s)}\left[\frac{\Gamma^{\prime}\left(b+\frac{r}{2}\right)}{\Gamma\left(b+\frac{r}{2}\right)}+\gamma\right]+\operatorname{PP} \zeta_{k, N}(r) .
$$

Finally, note by (5.39) and (5.40)

$$
\sum_{b=0}^{r}\left(z_{r, b}\left(-\alpha_{k}\right)-z_{r, b}\left(\alpha_{k}\right)\right)=M_{r}\left(1,-\alpha_{k}\right)-M_{r}\left(1, \alpha_{k}\right)=\frac{\left(-\alpha_{k}\right)^{r}-\alpha_{k}^{r}}{r} .
$$

Differentiating (5.57), we arrive at the result; note that $\epsilon$-dependence cancels, since (5.65) vanishes for $r$ even, whereas on the even dimensional closed Riemannian manifold $\left(N^{n}, g^{N}\right)$ the residue $\operatorname{Res}_{s=r} \zeta_{k, N}(s)$ vanishes for $r$ odd.

Proposition 5.15. Let $\left(E_{N}, \nabla_{N}, h_{N}\right)$ be a flat Hermitian vector bundle over an evendimensional oriented closed Riemannian manifold $\left(N^{n}, g^{N}\right)$. Denote the Euler characteristic of $\left(N, E_{N}\right)$ by $\chi\left(N, E_{N}\right)$ and the Betti numbers by $b_{k}:=\operatorname{dim} H^{k}\left(N, E_{N}\right)$. Then in notation of Definition 5.4 we find

$$
\begin{aligned}
\sum_{k=0}^{n} \frac{(-1)^{k+1}}{2} \zeta_{k, H}^{\prime}(0, \epsilon) & =\sum_{k=0}^{n} \frac{(-1)^{k}}{2} b_{k} \log \left(\frac{1-\epsilon^{n-2 k+1}}{n-2 k+1}\right) \\
& +\sum_{k=0}^{n / 2-1}(-1)^{k} b_{k} \sum_{l=0}^{n / 2-k-1} \log (2 l+1) \\
& +\sum_{k=0}^{n / 2-1} \frac{(-1)^{k}}{2} b_{k} \log (n-2 k+1)
\end{aligned}
$$


Proof. By Definition 5.4 we can write

$$
\begin{aligned}
\sum_{k=0}^{n} \frac{(-1)^{k+1}}{2} \zeta_{k, H}(s, \epsilon) & =\sum_{k=0}^{n} \frac{(-1)^{k+1}}{2} b_{k} \zeta\left(s, H_{0, \epsilon, \mathrm{rel}}^{k}\right) \\
& -\sum_{k=0}^{n} \frac{(-1)^{k+1}}{2} b_{k} \zeta\left(s, H_{0, \mathrm{rel}}^{k}\right)=: H(s, \epsilon)-H(s) .
\end{aligned}
$$

$H^{\prime}(0)$ has been evaluated in [VER09A, Theorem 7.8] with

$$
\begin{aligned}
\sum_{k=0}^{n} \frac{(-1)^{k+1}}{2} b_{k} \zeta^{\prime}\left(0, H_{0, \text { rel }}^{k}\right)= & \frac{\log 2}{2} \chi\left(N, E_{N}\right)-\sum_{k=0}^{n / 2-1} \frac{(-1)^{k}}{2} b_{k} \log (n-2 k+1) \\
& -\sum_{k=0}^{n / 2-1}(-1)^{k} b_{k} \sum_{l=0}^{n / 2-k-1} \log (2 l+1)
\end{aligned}
$$

We evaluate $H^{\prime}(0, \epsilon)$ using [LEs98, Theorem 1.2], which relates the zeta determinants to the normalized solutions of the operators, satisfying the corresponding boundary conditions. The boundary conditions for $H_{0, \epsilon, \text { rel }}^{k}$ have been determined in Proposition 5.3, and are given by the Dirichlet boundary conditions. By the formula [LES98, Theorem 1.2] we then find

$$
\operatorname{det}_{\zeta}\left(H_{0, \epsilon, \mathrm{rel}}^{k}\right)=\frac{\sqrt{\epsilon}}{\left|\alpha_{k}\right|}\left(\epsilon^{-\left|\alpha_{k}\right|}-\epsilon^{\left|\alpha_{k}\right|}\right)
$$

Taking logarithms and employing Poincare duality on $\left(N, g^{N}\right)$ we find

$$
\begin{aligned}
H^{\prime}(0, \epsilon) & =\sum_{k=0}^{n} \frac{(-1)^{k}}{2} b_{k} \log \operatorname{det}_{\zeta}\left(H_{0, \epsilon, \mathrm{rel}}^{k}\right) \\
& =\sum_{k=0}^{n} \frac{(-1)^{k}}{2} b_{k} \log \left(\frac{\sqrt{\epsilon}}{\left|\alpha_{k}\right|}\left(\epsilon^{-\left|\alpha_{k}\right|}-\epsilon^{\left|\alpha_{k}\right|}\right)\right) \\
& =\sum_{k=0}^{n} \frac{(-1)^{k}}{2} b_{k} \log \left(\frac{\sqrt{\epsilon}}{\left|\alpha_{k}+1\right|}\left(\epsilon^{-\left|\alpha_{k}+1\right|}-\epsilon^{\left|\alpha_{k}+1\right|}\right)\right) .
\end{aligned}
$$

Obviously, we can replace $\left|\alpha_{k}+1\right|$ by $\left(\alpha_{k}+1\right)$ in the expression above, and find after straightforward cancellations

$$
\begin{aligned}
H^{\prime}(0, \epsilon) & =\sum_{k=0}^{n} \frac{(-1)^{k}}{2} b_{k} \log \left(\frac{\sqrt{\epsilon}}{\left(\alpha_{k}+1\right)}\left(\epsilon^{-\left(\alpha_{k}+1\right)}-\epsilon^{\left(\alpha_{k}+1\right)}\right)\right) \\
& =\sum_{k=0}^{n} \frac{(-1)^{k}}{2} b_{k} \log \left(2 \epsilon^{k-n / 2}\left(\frac{1-\epsilon^{n-2 k+1}}{n-2 k+1}\right)\right) \\
& =\sum_{k=0}^{n} \frac{(-1)^{k}}{2} b_{k} \log \left(\frac{1-\epsilon^{n-2 k+1}}{n-2 k+1}\right)+\frac{\log 2}{2} \chi\left(N, E_{N}\right) .
\end{aligned}
$$

The statement follows by combination of (5.68) and (5.71). 
Summing up the expressions in Corollary 5.14 and Proposition 5.15, we arrive at the following result.

Theorem 5.16. Let $\left(C(N)=(0,1] \times N, g=d x^{2} \oplus x^{2} g^{N}\right)$ be an odd-dimensional bounded cone over a closed oriented Riemannian manifold $\left(N^{n}, g^{N}\right)$. Denote by $\left(C_{\epsilon}(N)=[\epsilon, 1] \times\right.$ $N, g)$ its truncation. Let $\left(E, \nabla, h^{E}\right)$ be a flat complex Hermitian vector bundle over $(C(N), g)$ and $\left(E_{N}, \nabla_{N}, h_{N}\right)$ its restriction to the cross-section $N$. Denote by $\chi\left(N, E_{N}\right)$ the Euler characteristic and by $b_{k}:=\operatorname{dim} H^{k}\left(N, E_{N}\right)$ the Betti numbers of $\left(N, E_{N}\right)$. Denote by $\Delta_{k, c c l, N}$ the Laplacian on coclosed $k$-differential forms $\Omega_{\mathrm{ccl}}^{k}\left(N, E_{N}\right)$ and put

$$
\begin{aligned}
& \alpha_{k}:=\frac{(n-1)}{2}-k, \quad \nu(\eta)=\sqrt{\eta+\alpha_{k}^{2}}, \text { for } \eta \in E_{k}=\operatorname{Spec} \Delta_{k, c c l, N} \backslash\{0\} \\
& \zeta_{k, N}(s)=\sum_{\eta \in E_{k}} \mathrm{~m}(\eta) \nu(\eta)^{-s}, \quad \zeta_{k, N}\left(s, \pm \alpha_{k}\right):=\sum_{\eta \in E_{k}} \mathrm{~m}(\eta)\left(\nu(\eta) \pm \alpha_{k}\right)^{-s}, \quad \operatorname{Re}(s) \gg 0
\end{aligned}
$$

where $\mathrm{m}(\eta)$ denotes the multiplicity of $\eta \in E_{k}$. Then the difference of the scalar analytic torsions for $(C(N), g)$ and $\left(C_{\epsilon}(N), g\right)$ is given by the following explicit expression

$$
\begin{aligned}
& \log T\left(C_{\epsilon}(N), E, g\right)-\log T(C(N), E, g)=\sum_{k=0}^{n} \frac{(-1)^{k}}{2} b_{k} \log \left(\frac{1-\epsilon^{n-2 k+1}}{n-2 k+1}\right) \\
& +\sum_{k=0}^{n / 2-1}(-1)^{k} b_{k} \sum_{l=0}^{n / 2-k-1} \log (2 l+1)+\sum_{k=0}^{n / 2-1} \frac{(-1)^{k}}{2} b_{k} \log (n-2 k+1) \\
& +\sum_{k=0}^{n / 2-1} \frac{(-1)^{k}}{4} \sum_{r=1}^{n / 2} \operatorname{Res}_{s=2 r} \zeta_{k, N}(s) \sum_{b=0}^{2 r}\left(z_{2 r, b}\left(-\alpha_{k}\right)-z_{2 r, b}\left(\alpha_{k}\right)\right) \frac{\Gamma^{\prime}(b+r)}{\Gamma(b+r)} \\
& +\sum_{k=0}^{n / 2-1} \frac{(-1)^{k}}{2}\left(\zeta_{k, N}^{\prime}\left(0,-\alpha_{k}\right)-\zeta_{k, N}^{\prime}\left(0, \alpha_{k}\right)\right) .
\end{aligned}
$$

Comparison of Theorem 5.16 and Theorem 2.6 yields the following

Corollary 5.17. Let $\left(C_{\epsilon}(N)=[\epsilon, 1] \times N, g, g_{0}\right), \epsilon>0$, be an odd-dimensional cylinder over a closed Riemannian manifold $\left(N, g^{N}\right)$, with a pair of Riemannian metrics $g=$ $d x^{2} \oplus x^{2} g^{N}$ and $g_{0}=d x^{2} \oplus g^{N}$. The Riemannian manifold $\left(C_{\epsilon}(N), g\right)$ is a truncated cone, while $\left(C_{\epsilon}(N), g_{0}\right)$ is an exact cylinder. Fix a flat complex Hermitian vector bundle $\left(E, \nabla, h^{E}\right)$. Then

$$
\begin{aligned}
& \log T\left(C_{\epsilon}(N), E, g\right)=\sum_{k=0}^{n} \frac{(-1)^{k}}{2} b_{k} \log \left(\frac{1-\epsilon^{n-2 k+1}}{n-2 k+1}\right)+\frac{\log 2}{2} \chi\left(N, E_{N}\right) \\
& +\sum_{k=0}^{n / 2-1} \frac{(-1)^{k}}{2} \sum_{r=1}^{n / 2} \operatorname{Res}_{s=2 r} \zeta_{k, N}(s) \sum_{b=0}^{2 r}\left(z_{2 r, b}\left(-\alpha_{k}\right)-z_{2 r, b}\left(\alpha_{k}\right)\right) \frac{\Gamma^{\prime}(b+r)}{\Gamma(b+r)},
\end{aligned}
$$


and the analytic torsion norms of $\left(C_{\epsilon}(N), g\right)$ and $\left(C_{\epsilon}(N), g_{0}\right)$ are related as follows

$$
\begin{aligned}
\log \left(\frac{\|\cdot\|_{\left(C_{\epsilon}(N), E, g\right)}^{R S}}{\|\cdot\|_{\left(C_{\epsilon}(N), E, g_{0}\right)}^{R S}}\right) & =\sum_{k=0}^{n / 2-1} \frac{(-1)^{k}}{2} \sum_{r=1}^{n / 2} \operatorname{Res}_{s=2 r} \zeta_{k, N}(s) \\
& \times \sum_{b=0}^{2 r}\left(z_{2 r, b}\left(-\alpha_{k}\right)-z_{2 r, b}\left(\alpha_{k}\right)\right) \frac{\Gamma^{\prime}(b+r)}{\Gamma(b+r)} .
\end{aligned}
$$

Proof. The first relation (5.72) follows by a direct comparison of Theorem 5.16 and Theorem 2.6. For the second relation note, that by definition of analytic torsion norms we have by $(5.72)$

$$
\begin{aligned}
\log \left(\frac{\|\cdot\|_{\left(C_{\epsilon}(N), E ; g\right)}^{R S}}{\|\cdot\|_{\left(C_{\epsilon}(N), E ; g_{0}\right)}^{R S}}\right) & =\sum_{k=0}^{n} \frac{(-1)^{k}}{2} b_{k} \log \left(\frac{1-\epsilon^{n-2 k+1}}{n-2 k+1}\right)-\frac{1}{2} \chi\left(N, E_{N}\right) \log (1-\epsilon) \\
& +\sum_{k=0}^{n / 2-1} \frac{(-1)^{k}}{2} \sum_{r=1}^{n / 2} \operatorname{Res}_{s=2 r} \zeta_{k, N}(s) \sum_{b=0}^{2 r}\left(z_{2 r, b}\left(-\alpha_{k}\right)-z_{2 r, b}\left(\alpha_{k}\right)\right) \frac{\Gamma^{\prime}(b+r)}{\Gamma(b+r)} \\
& +\log \left(\frac{\|\cdot\|_{\operatorname{det} H^{*}\left(C_{\epsilon}(N), E\right), g}}{\|\cdot\|_{\operatorname{det} H^{*}\left(C_{\epsilon}(N), E\right), g_{0}}}\right)
\end{aligned}
$$

The quotient between the norms on $\operatorname{det} H^{*}\left(C_{\epsilon}(N), E\right)$, induced by the $L^{2}\left(g, h^{E}\right)$ and $L^{2}\left(g_{0}, h^{E}\right)$ norms on harmonic forms, amounts by a straightforward computation to $\log \left(\frac{\|\cdot\|_{\operatorname{det} H^{*}\left(C_{\epsilon}(N), E\right), g}}{\|\cdot\|_{\operatorname{det} H^{*}\left(C_{\epsilon}(N), E\right), g_{0}}}\right)=\frac{1}{2} \chi\left(N, E_{N}\right) \log (1-\epsilon)-\sum_{k=0}^{n} \frac{(-1)^{k}}{2} b_{k} \log \left(\frac{1-\epsilon^{n-2 k+1}}{n-2 k+1}\right)$.

Hence overall we arrive at the following

$\log \left(\frac{\|\cdot\|_{\left(C_{\epsilon}(N), E ; g\right)}^{R S}}{\|\cdot\|_{\left(C_{\epsilon}(N), E ; g_{0}\right)}^{R S}}\right)=\sum_{k=0}^{n / 2-1} \frac{(-1)^{k}}{2} \sum_{r=1}^{n / 2} \operatorname{Res}_{s=2 r} \zeta_{k, N}(s) \sum_{b=0}^{2 r}\left(z_{2 r, b}\left(-\alpha_{k}\right)-z_{2 r, b}\left(\alpha_{k}\right)\right) \frac{\Gamma^{\prime}(b+r)}{\Gamma(b+r)}$.

\section{Metric Anomaly at the Regular Boundary of the Cone}

Consider a truncated cone $C_{\epsilon}(N)=[\epsilon, 1] \times N, g=d x^{2} \oplus x^{2} g^{N}$ over a closed oriented Riemannian manifold $\left(N^{n}, g^{N}\right)$. The Levi-Civita connection $\nabla^{T C_{\epsilon}(N)}$, induced by $g$, defines secondary classes $B_{\epsilon}\left(\nabla^{T C_{\epsilon}(N)}\right)$ and $B_{1}\left(\nabla^{T C_{\epsilon}(N)}\right)$ at the left $\{x=\epsilon\} \times N$ and the right $\{x=1\} \times N$ boundary components of $C_{\epsilon}(N)$, respectively.

Introducing new coordinates $y=\log (1 / x)$ near the right boundary component $\{x=$ $1\} \times N$ of $C_{\epsilon}(N)$, and $z=\log (x / \epsilon)$ near the left boundary component $\{x=\epsilon\} \times N$, we can write for $\delta>0$ small the Riemannian metric $g$ as follows

$$
\begin{aligned}
& g=e^{-2 y}\left(d y^{2}+g^{N}\right), y \in[0, \delta), \text { near }\{x=1\} \times N \text { of } C_{\epsilon}(N), \\
& g=\epsilon^{2} e^{2 z}\left(d z^{2}+g^{N}\right), z \in[0, \delta), \text { near }\{x=\epsilon\} \times N \text { of } C_{\epsilon}(N) .
\end{aligned}
$$

By Proposition 4.1 and in view of (4.4) and the explicit formulae in (4.2), we deduce

$$
B_{\epsilon}\left(\nabla^{T C_{\epsilon}(N)}\right)=B_{1}\left(\nabla^{T C_{\epsilon}(N)}\right)=: B_{1}\left(g^{N}\right),
$$


independent of $\epsilon>0$. Note, that the metric anomalies at $x=\epsilon$ and $x=1$ are defined with respect to different inward unit normal vectors at the boundary, which amounts to an additional sign only in case of an odd-dimensional cross section. In our setup of even-dimensional cross-section, both anomalies coincide. Since the corresponding secondary classes induced by the product metric on $C_{\epsilon}(N)$ are zero, we arrive by (2.16) at the following proposition.

Proposition 6.1. Let $\left(C_{\epsilon}(N)=[\epsilon, 1] \times N, g, g_{0}\right), \epsilon>0$, be an odd-dimensional cylinder over a closed Riemannian manifold $\left(N, g^{N}\right)$, with a pair of Riemannian metrics $g=$ $d x^{2} \oplus x^{2} g^{N}$ and $g_{0}=d x^{2} \oplus g^{N}$. The Riemannian manifold $\left(C_{\epsilon}(N), g\right)$ is a truncated cone, while $\left(C_{\epsilon}(N), g_{0}\right)$ is an exact cylinder. Fix a flat complex Hermitian vector bundle $\left(E, \nabla, h^{E}\right)$. Then the analytic torsion norms are related as follows

$$
\log \left(\frac{\|\cdot\|_{\left(C_{\epsilon}(N), E ; g\right)}^{R S}}{\|\cdot\|_{\left(C_{\epsilon}(N), E ; g_{0}\right)}^{R S}}\right)=-\operatorname{rank}(E) \int_{N} B_{1}\left(g^{N}\right) .
$$

Comparing Corollary 5.17 and Proposition 6.1, we arrive at the following result.

Corollary 6.2. Let $\left(C(N) \cong(0,1) \times N, g=d x^{2} \oplus x^{2} g^{N}\right)$ be an odd-dimensional bounded cone over a closed oriented Riemannian manifold $\left(N^{n}, g^{N}\right)$. Let $\left(E, \nabla, h^{E}\right)$ be a flat complex Hermitian vector bundle and $\left(E_{N}, \nabla_{N}, h_{N}\right)$ its restriction to the crosssection $N$ over $C(N)$. Then, in the notation of Section 5, the integral of the secondary class $B_{1}\left(g^{N}\right)$ may be expressed as follows

$\operatorname{rank}(E) \int_{N} B_{1}\left(g^{N}\right)=\sum_{k=0}^{n / 2-1} \frac{(-1)^{k+1}}{2} \sum_{r=1}^{n / 2} \operatorname{Res}_{s=2 r} \zeta_{k, N}(s) \sum_{b=0}^{2 r}\left(z_{2 r, b}\left(-\alpha_{k}\right)-z_{2 r, b}\left(\alpha_{k}\right)\right) \frac{\Gamma^{\prime}(b+r)}{\Gamma(b+r)}$.

Remark 6.3. The quotient of analytic torsion norms in (5.73) is independent of $\epsilon>$ 0 , which also implies scaling invariance of the secondary class $B_{1}\left(\nabla^{T C_{\epsilon}(N)}\right)$ and the statement of Proposition 6.1 follows, independently of the general result in Proposition 4.1.

Example 6.4. The following example might be illuminating for the identity in Corollary 6.2. Consider the special case of $N=T^{2}$ being the two-dimensional flat torus, and $E$ a trivial line bundle. Then one can verify Corollary 6.2 by direct computations. Indeed, from [BGKE96, (3.6), (3.7)] we infer

$$
M_{2}(t, \alpha)=\sum_{b=0}^{1} z_{2, b}(\alpha) t^{2+2 b}=\left(-\frac{3}{16}+\frac{\alpha}{2}-\frac{\alpha^{2}}{2}\right) t^{2}+\left(\frac{5}{8}-\frac{\alpha}{2}\right) t^{4}-\frac{7}{16} t^{6} .
$$

Consequently, with $n=2$ and $\alpha_{0}=1 / 2$, we find

$$
\begin{aligned}
& \left(z_{2,0}(-1 / 2)-z_{2,0}(1 / 2)\right) \frac{\Gamma^{\prime}(1)}{\Gamma(1)}=-\frac{1}{2} \frac{\Gamma^{\prime}(1)}{\Gamma(1)}=\frac{\gamma}{2} \\
& \left(z_{2,1}(-1 / 2)-z_{2,1}(1 / 2)\right) \frac{\Gamma^{\prime}(2)}{\Gamma(2)}=\frac{1}{2} \frac{\Gamma^{\prime}(2)}{\Gamma(2)}=\frac{1}{2}(1-\gamma), \\
& \left(z_{2,2}(-1 / 2)-z_{2,2}(1 / 2)\right) \frac{\Gamma^{\prime}(3)}{\Gamma(3)}=0 .
\end{aligned}
$$


Moreover, the heat trace expansion for the flat two-dimensional torus implies

$$
\operatorname{Res}_{s=2} \zeta_{0, N}(s)=2 \cdot \operatorname{Res}_{s=1} \zeta\left(s, \Delta_{0, N}\right)=2 \cdot(4 \pi)^{-1} \operatorname{Vol}\left(T^{2}\right) .
$$

In total we find for the right hand side of the equality in Corollary 6.2

$$
\begin{gathered}
\sum_{k=0}^{n / 2-1} \frac{(-1)^{k+1}}{2} \sum_{r=1}^{n / 2} \operatorname{Res}_{s=2 r} \zeta_{k, N}(s) \sum_{b=0}^{2 r}\left(z_{2 r, b}\left(-\alpha_{k}\right)-z_{2 r, b}\left(\alpha_{k}\right)\right) \frac{\Gamma^{\prime}(b+r)}{\Gamma(b+r)} \\
=-\frac{1}{8 \pi} \operatorname{Vol}\left(T^{2}\right) .
\end{gathered}
$$

On the other hand, the expression for the integral of $B_{1}\left(g^{N}\right)$ follows from [BRMA06, (4.43)], which in our special case reduces to

$$
\int_{N} B_{1}\left(g^{N}\right)=-\frac{1}{2 \pi}\left(\frac{1}{2}\right)^{2} \operatorname{Vol}\left(T^{2}\right)=-\frac{1}{8 \pi} \operatorname{Vol}\left(T^{2}\right) .
$$

Both, (6.6) and (6.7) agree, as asserted by Corollary 6.2.

Corollary 6.2 together with Theorem 2.6 leads to the main result of this section, announced in Theorem 2.8.

Theorem 6.5. Let $\left(C(N) \cong(0,1) \times N, g=d x^{2} \oplus x^{2} g^{N}\right)$ be an odd-dimensional bounded cone over a closed oriented Riemannian manifold $\left(N^{n}, g^{N}\right)$. Let $\left(E, \nabla, h^{E}\right)$ be a flat complex Hermitian vector bundle and $\left(E_{N}, \nabla_{N}, h_{N}\right)$ its restriction to the cross-section $N$ over $C(N)$. Let $b_{k}=\operatorname{dim} H^{k}\left(N, E_{N}\right)$ be the Betti numbers and $\chi\left(N, E_{N}\right)$ the Euler characteristic of $\left(N, E_{N}\right)$. Denote by $\Delta_{k, c c l, N}$ the Laplacian on coclosed $k$-differential forms $\Omega_{\mathrm{ccl}}^{k}\left(N, E_{N}\right)$. Put $\alpha_{k}=(n-1) / 2-k$ and define

$$
\begin{aligned}
& \nu(\eta)=\sqrt{\eta+\alpha_{k}^{2}}, \text { for } \eta \in E_{k}=\operatorname{Spec} \Delta_{k, c c l, N} \backslash\{0\}, \\
& \zeta_{k, N}\left(s, \pm \alpha_{k}\right):=\sum_{\eta \in E_{k}} \operatorname{m}(\eta)\left(\nu(\eta) \pm \alpha_{k}\right)^{-s}, \quad \operatorname{Re}(s) \gg 0,
\end{aligned}
$$

where $\mathrm{m}(\eta)$ denotes the multiplicity of $\eta \in E_{k}$. Then the logarithm of the scalar analytic torsion of $(C(N), g)$, is given by

$$
\begin{aligned}
\log T(C(N), E, g) & =\sum_{k=0}^{n / 2-1} \frac{(-1)^{k+1}}{2} b_{k}\left(\sum_{l=0}^{n / 2-k-1} \log (2 l+1)^{2}+\log (n-2 k+1)\right) \\
& +\sum_{k=0}^{n / 2-1} \frac{(-1)^{k}}{2}\left(\zeta_{k, N}^{\prime}\left(0, \alpha_{k}\right)-\zeta_{k, N}^{\prime}\left(0,-\alpha_{k}\right)\right) \\
& +\frac{\log 2}{2} \chi\left(N, E_{N}\right)-\frac{1}{2} \operatorname{rank}(E) \int_{N} B_{1}\left(g^{N}\right) .
\end{aligned}
$$

Theorem 6.5 identifies the residual term in the formula for analytic torsion of a bounded cone in Theorem 2.6 in terms of the metric anomaly of analytic torsion at the regular boundary of the cone. This identifies the actual contribution of the conical singularity to the analytic torsion, clearing up the formula in Theorem 2.6 of the 
contributions from the regular boundary. We can rewrite Theorem 6.5 in terms of the analytic torsion norm.

Corollary 6.6. Let $C(N)=(0,1] \times N, g=d x^{2} \oplus x^{2} g^{N}$ be an odd-dimensional bounded cone over a closed oriented Riemannian manifold $\left(N^{n}, g^{N}\right)$. Consider $\left(C(N), g_{0}\right)$, where $g_{\mathrm{pr}}$ coincides with $g$ near the singularity at $x=0$ and is product $d x^{2} \oplus g^{N}$ near the boundary $\{x=1\} \times N$. Let $\left(E, \nabla, h^{E}\right)$ be a flat complex Hermitian vector bundle and $\left(E_{N}, \nabla_{N}, h_{N}\right)$ its restriction to the cross-section $N$ over $C(N)$. Then the quotient of analytic torsion norm for $\left(C(N), g_{\mathrm{pr}}\right)$ and the $L^{2}\left(g, h^{E}\right)$-induced norm on $\operatorname{det} H^{k}(C(N), E)$ is given by

$$
\begin{aligned}
\log \left(\frac{\|\cdot\|_{\left(C(N), E ; g_{\mathrm{pr}}\right)}^{R S}}{\|\cdot\|_{\operatorname{det} H^{k}(C(N), E), g}}\right) & =\sum_{k=0}^{n / 2-1}(-1)^{k+1} \frac{b_{k}}{2}\left(\sum_{l=0}^{n / 2-k-1} \log (2 l+1)^{2}+\log (n-2 k+1)\right) \\
& +\frac{\log 2}{2} \chi\left(N, E_{N}\right)+\sum_{k=0}^{n / 2-1} \frac{(-1)^{k}}{2}\left(\zeta_{k, N}^{\prime}\left(0, \alpha_{k}\right)-\zeta_{k, N}^{\prime}\left(0,-\alpha_{k}\right)\right) .
\end{aligned}
$$

Proof. The metric variation for analytic torsion is local by the gluing formula in [LES10] and hence the metric anomaly formula of Brüning-Ma in[BrMA06] holds also in case of manifolds with isolated conical singularities away from the variation region, so that (2.16) applies to $\left(C(N), g, g_{\mathrm{pr}}\right)$.

$$
\begin{aligned}
\log \left(\frac{\|\cdot\|_{\left(C(N), E ; g_{\mathrm{pr}}\right)}^{R S}}{\|\cdot\|_{\operatorname{det} H^{k}(C(N), E), g}}\right) & =\log \left(\frac{\|\cdot\|_{\left(C(N), E ; g_{\mathrm{pr}}\right)}^{R S}}{\|\cdot\|_{(C(N), E ; g)}^{R S}}\right)+\log T(C(N), E ; g) \\
& =\log T(C(N), E ; g)+\frac{1}{2} \operatorname{rank}(E) \int_{N} B_{1}\left(g^{N}\right) .
\end{aligned}
$$

The claim follows by Theorem 6.5.

\section{Asymptotics of the New Torsion-Like Spectral Invariant}

Analytic torsion defines a topological invariant of an odd-dimensional closed oriented Riemannian manifold with a flat Hermitian vector bundle. In even dimensions, analytic torsion is trivial as a consequence of Poincare duality. Theorem 6.5 identifies the contribution of a conical singularity to analytic torsion in terms of a new torsion-like spectral invariant of the even-dimensional cross-section, which is non-trivial despite Poincare duality and deserves an independent definition.

Definition 7.1. Let $\left(N^{n}, g^{N}\right)$ be an even-dimensional closed oriented Riemannian manifold and $\left(E_{N}, \nabla_{N}, h\right)$ a flat flat complex Hermitian vector bundle. Denote by $\Delta_{k, c c l, N}$ the corresponding Laplacian on coclosed $k$-differential forms $\Omega_{\mathrm{ccl}}^{k}\left(N, E_{N}\right)$. Put $\alpha_{k}=(n-1) / 2-k$ and define

$$
\begin{aligned}
& \nu(\eta)=\sqrt{\eta+\alpha_{k}^{2}}, \text { for } \eta \in E_{k}=\operatorname{Spec} \Delta_{k, c c l, N} \backslash\{0\} \\
& \zeta_{k, N}\left(s, \pm \alpha_{k}\right):=\sum_{\eta \in E_{k}} \operatorname{m}(\eta)\left(\nu(\eta) \pm \alpha_{k}\right)^{-s}, \quad \operatorname{Re}(s) \gg 0
\end{aligned}
$$


where $\mathrm{m}(\eta)$ denotes the multiplicity of $\eta \in E_{k}$. Then the "torsion-like" invariant $\operatorname{Tors}\left(N, E_{N} ; g^{N}\right)$ is defined as

$\operatorname{Tors}\left(N, E_{N} ; g^{N}\right):=\frac{1}{2} \sum_{k=0}^{n / 2-1}(-1)^{k}\left(\zeta_{k, N}^{\prime}\left(0, \alpha_{k}\right)-\zeta_{k, N}^{\prime}\left(0,-\alpha_{k}\right)\right)=\frac{1}{2} \sum_{k=0}^{n-1}(-1)^{k} \zeta_{k, N}^{\prime}\left(0, \alpha_{k}\right)$

We study the asymptotic behavior of $\operatorname{Tors}\left(N, E_{N} ; g^{N}\right)$ under scaling of $g^{N}$, employing the perturbation analysis by Gambia-Mushiest-Solo min in [GSMS83]. Let $A$ be an elliptic non-negative self-adjoint differential operator of second order over a closed Riemannian manifold $\left(N, g^{N}\right)$ acting on sections of a Hermitian vector bundle $E$. Consider the square root $A_{\alpha}:=\sqrt{A+\alpha^{2}}$, which is a self-adjoint pseudo-differential operator of first order. Following the arguments of [GSMS83, Corollary 1] we arrive at the following result.

Theorem 7.2. The zeta-function of $\left(A_{\alpha}+\alpha\right)$ is analytic for $\operatorname{Re}(s)>-(\operatorname{dim} N+2)$ with the following expansion ( $K=\operatorname{dim} N+2$ )

$$
\zeta\left(s, A_{\alpha}+\alpha\right)=\zeta\left(s, A_{\alpha}\right)+\sum_{k=1}^{K-1} \alpha^{k} \mathscr{T}_{k}(s, \alpha)+\alpha^{K} \mathscr{R}_{K}(s, \alpha)
$$

where $\mathscr{R}_{K}(s, \alpha)$ is holomorphic and $\mathscr{R}_{K}, \partial_{s} \mathscr{R}_{K}$ are locally uniformly bounded in $\alpha$, and

$$
\mathscr{T}_{k}(s, \alpha)=\frac{(-1)^{k}}{k !} \zeta\left(s+k, A_{\alpha}\right) \prod_{j=0}^{k-1}(s+j)
$$

We now can prove the main result of this subsection.

Corollary 7.3. The zeta-functions of $\left(A_{\alpha} \pm \alpha\right)$ are regular at $s=0$ and

$$
\left.\frac{d}{d s}\right|_{0} \zeta\left(s, A_{\alpha}+\alpha\right)-\left.\frac{d}{d s}\right|_{0} \zeta\left(s, A_{\alpha}-\alpha\right)=O(\alpha), \quad \alpha \rightarrow 0
$$

Proof. Theorem 7.2 implies for $K=\operatorname{dim} N+2$

$$
\left.\zeta\left(s, A_{\alpha}+\alpha\right)-\zeta\left(s, A_{\alpha}-\alpha\right)=\sum_{k=0, \text { odd }}^{K-1} 2 \alpha^{k} \mathscr{T}_{k}(s, \alpha)+\alpha^{K}\left(\mathscr{R}_{K}(s, \alpha)-\mathscr{R}_{K}(s,-\alpha)\right)\right)
$$

The term $\left.\left(\mathscr{R}_{K}(s, \alpha)-\mathscr{R}_{K}(s,-\alpha)\right)\right)$, and also its $\partial_{s}$-differential, are both regular at $s=0$ and locally uniformly bounded in $\alpha \in \mathbb{R}$. Hence it remains to analyze the terms $\mathscr{T}_{k}(s, \alpha)$. Repeating the arguments of Theorem 7.2 now for $\left(A+\alpha^{2}\right)$ as a perturbation 
of $A$, we find

$$
\begin{aligned}
\mathscr{T}_{k}(s, \alpha) & =\zeta\left(\frac{(s+k)}{2}, A+\alpha^{2}\right)\left(\frac{(-1)^{k}}{k !} \prod_{j=0}^{k-1}(s+j)\right) \\
& =\left(\frac{(-1)^{k}}{k !} \prod_{j=0}^{k-1}(s+j)\right)\left[\zeta\left(\frac{(s+k)}{2}, A\right)+\sum_{p=1}^{F-1} \alpha^{2 p} \zeta\left(\frac{(s+k)}{2}+p, A\right)\right. \\
& \times\left(\frac{(-1)^{p}}{p !} \prod_{j=0}^{p-1}\left(\left(\frac{(s+k)}{2}+j\right)\right)+\widehat{\mathscr{R}}_{F}((s+n) / 2, \alpha)\right]
\end{aligned}
$$

Again, the term $\widehat{\mathscr{R}}_{F}$ and also its $\partial_{s}$-differential, are both regular at $s=0$ and locally uniformly bounded in $\alpha \in \mathbb{R}$. The zeta-functions $\zeta((s+k) / 2+j, A)$ are meromorphic possibly with simple poles at $s=0$, canceled by the additional $s$-factor in (7.1), so that $\mathscr{T}_{k}(s, \alpha)$ is regular at $s=0$ and its derivative at $s=0$ is locally uniformly bounded in $\alpha \in \mathbb{R}$. The statement now follows.

We close the section by deriving the scaling behavior of the torsion-like invariant (Theorem 2.12), combining of Theorem 7.2 and Corollary 7.3.

Theorem 7.4. Let $\left(N^{n}, g^{N}\right)$ be a closed oriented Riemannian manifold of even dimension and $\left(E_{N}, \nabla_{N}, h\right)$ a flat complex Hermitian vector bundle. Let $g^{N}(\mu):=\mu^{-2} g^{N}, \mu \in$ $\mathbb{R}^{+}$and denote by $\Delta_{k, c c l, N}(\mu)$ the corresponding Laplacian on coclosed $k$-differential forms $\Omega_{\mathrm{ccl}}^{k}\left(N, E_{N}\right)$. Put $\alpha_{k}=(n-1) / 2-k$ and define

$$
\begin{aligned}
& \nu(\eta)=\sqrt{\eta+\alpha_{k}^{2}}, \text { for } \eta \in E_{k}=\operatorname{Spec} \Delta_{k, c c l, N} \backslash\{0\} \\
& \zeta_{k, N}\left(s, \pm \alpha_{k}\right):=\sum_{\eta \in E_{k}} \operatorname{m}(\eta)\left(\nu(\eta) \pm \alpha_{k}\right)^{-s}, \quad \operatorname{Re}(s) \gg 0
\end{aligned}
$$

where $\mathrm{m}(\eta)$ denotes the multiplicity of $\eta \in E_{k}$. Then the associated family of "torsionlike" invariants $\operatorname{Tors}\left(N, E_{N} ; g^{N}(\mu)\right)$ admits the following asymptotic behavior as $\mu \rightarrow \infty$

$$
\operatorname{Tors}\left(N, E_{N} ; g^{N}(\mu)\right)=\frac{1}{2} \sum_{k=0}^{n / 2-1}(-1)^{k}\left(\zeta_{k, N}^{\prime}\left(0, \alpha_{k}, \mu\right)-\zeta_{k, N}^{\prime}\left(0,-\alpha_{k}, \mu\right)\right)=O\left(\frac{\log \mu}{\mu}\right)
$$

Proof. Write $A$ for the coclosed Laplacian on $\Omega_{\mathrm{ccl}}^{k}\left(N, E_{N}\right)$ associated to $g^{N}$. Put $\alpha(\mu):=$ $\alpha_{k} \mu^{-1}$ and consider $A_{\alpha(\mu)}$ in the notation as fixed before. By definition, $\Delta_{k, c c l, N}(\mu)=$ $\mu^{2} A$, and consequently, $\operatorname{Spec} \Delta_{k, c c l, N}(\mu)=\mu^{2} \operatorname{Spec} A$. Hence

$$
\zeta_{k, N}\left(s, \pm \alpha_{k}, \mu\right)=\mu^{-s} \zeta\left(s, A_{\alpha(\mu)} \pm \alpha(\mu)\right) .
$$

Taking derivatives at $s=0$ we obtain

$$
\left.\frac{d}{d s}\right|_{s=0} \zeta_{k, N}\left(0, \pm \alpha_{k}, \mu\right)=-\log \mu \cdot \zeta\left(0, A_{\alpha(\mu)} \pm \alpha(\mu)\right)+\zeta^{\prime}\left(0, A_{\alpha(\mu)} \pm \alpha(\mu)\right) .
$$


Theorem 7.2 and Corollary 7.3 assert that as $\mu \rightarrow \infty$

$$
\begin{gathered}
\zeta^{\prime}\left(0, A_{\alpha(\mu)}+\alpha(\mu)\right)-\zeta^{\prime}\left(0, A_{\alpha(\mu)}-\alpha(\mu)\right)=O\left(\frac{1}{\mu}\right), \\
\zeta\left(0, A_{\alpha(\mu)}+\alpha(\mu)\right)-\zeta\left(0, A_{\alpha(\mu)}-\alpha(\mu)\right)=O\left(\frac{1}{\mu}\right) .
\end{gathered}
$$

This proves the statement.

\section{REFERENCES}

[AbSt92] M. Abramowitz and I. A. Stegun (eds.), Handbook of mathematical functions with formulas, graphs, and mathematical tables, Dover Publications Inc., New York, 1992, Reprint of the 1972 edition. MR 1225604 (94b:00012)

[BFK95] D. Burghelea, L. Friedlander, and T. Kappeler, On the determinant of elliptic boundary value problems on a line segment, Proc. Amer. Math. Soc. 123 (1995), no. 10, 3027-3038. MR 1301012 (95m:58131)

[BGKe96] M. Bordag, B. Geyer, K. Kirsten, and E. Elizalde, Zeta function determinant of the Laplace operator on the D-dimensional ball, Comm. Math. Phys. 179 (1996), no. 1, 215-234. MR 1395222 (97e:58226)

[BKD96] M. Bordag, K. Kirsten, and S. Dowker, Heat-kernels and functional determinants on the generalized cone, Comm. Math. Phys. 182 (1996), no. 2, 371-393. MR 1447298 (98d:58193)

[BrLe93] J. Brüning and M. Lesch, Kähler-Hodge theory for conformal complex cones, Geom. Funct. Anal. 3 (1993), no. 5, 439-473. MR 1233862 (94i:58189)

[BRMa06] J. BRÜNING and X. MA, An anomaly formula for Ray-Singer metrics on manifolds with boundary, Geom. Funct. Anal. 16 (2006), no. 4, 767-837. MR 2255381 (2007i:58042)

[BrSe85] J. Brüning and R. Seeley, Regular singular asymptotics, Adv. in Math. 58 (1985), no. 2, 133-148. MR 814748 (87b:41032)

[BRSE88] _ An index theorem for first order regular singular operators, Amer. J. Math. 110 (1988), no. 4, 659-714. MR 955293 (89k:58271)

[Che79A] J. Cheeger, Analytic torsion and the heat equation, Ann. of Math. (2) 109 (1979), no. 2, 259-322. MR 528965 (80j:58065a)

[CHE79B] On the spectral geometry of spaces with cone-like singularities, Proc. Nat. Acad. Sci. U.S.A. 76 (1979), no. 5, 2103-2106. MR 530173 (80k:58098)

[CHE83] _ Spectral geometry of singular Riemannian spaces, J. Differential Geom. 18 (1983), no. 4, 575-657 (1984). MR 730920 (85d:58083)

[DAFA00] X. DAI and H. FAng, Analytic torsion and R-torsion for manifolds with boundary, Asian J. Math. 4 (2000), no. 3, 695-714. MR 1796700 (2001j:58057)

[DAR87] A. DAR, Intersection R-torsion and analytic torsion for pseudomanifolds, Math. Z. 194 (1987), no. 2, 193-216. MR 876230 (88b:58132)

[DMHS09] T. De Melo, L. Hartmann, and M. Spreafico, Reidemeister torsion and analytic torsion of discs, Boll. Unione Mat. Ital. (9) 2 (2009), no. 2, 529-533. MR 2537286 (2010f:58043)

[GrRy94] I. S. Gradshteyn and I. M. Ryzhik, Table of integrals, series, and products, russian ed., Academic Press Inc., Boston, MA, 1994, Translation edited and with a preface by Alan Jeffrey. MR 1243179 (94g:00008)

[GSMS83] R. E. Gamboa Saraví, M. A. Muschietti, and J. E. Solomin, On perturbation theory for regularized determinants of differential operators, Comm. Math. Phys. 89 (1983), no. 3, 363-373. MR 709472 (85i:81046)

[HaSp10] L. Hartmann and M. Spreafico, The analytic torsion of a cone over a sphere, J. Math. Pures Appl. (9) 93 (2010), no. 4, 408-435. MR 2609036 
[Les94] M. Lesch, The Analytic Torsion of the Model Cone, unpublished notes (1994).

[LES97] _ Operators of Fuchs type, conical singularities, and asymptotic methods, TeubnerTexte zur Mathematik [Teubner Texts in Mathematics], vol. 136, B. G. Teubner Verlagsgesellschaft mbH, Stuttgart, 1997. MR 1449639 (98d:58174)

[LEs98] _ Determinants of regular singular Sturm-Liouville operators, Math. Nachr. 194 (1998), 139-170. MR 1653090 (99j:58220)

[LES10] Gluing formula for analytic torsion, in preparation.

[L̈̈C93] W. LüCK, Analytic and topological torsion for manifolds with boundary and symmetry, J. Differential Geom. 37 (1993), no. 2, 263-322. MR 1205447 (94e:57054)

[MaVe10] R. MazzeO and B. Vertman, Analytic torsion on manifolds with edges, arXiv:1103.0448 [math.SP].

[Mel92] R. B. Melrose, Calculus of conormal distributions on manifolds with corners, Internat. Math. Res. Notices (1992), no. 3, 51-61. MR 1154213 (93i:58148)

[Mel93] ㄴ The Atiyah-Patodi-Singer index theorem, Research Notes in Mathematics, vol. 4, A K Peters Ltd., Wellesley, MA, 1993. MR 1348401 (96g:58180)

[Moo99] E. A. Mooers, Heat kernel asymptotics on manifolds with conic singularities, J. Anal. Math. 78 (1999), 1-36. MR 1714065 (2000g:58039)

[Mül78] W. Müller, Analytic torsion and R-torsion of riemannian manifolds, Adv. in Math. 28 (1978), no. 3, 233-305. MR 498252 (80j:58065b)

[Olv97] F. W. J. Olver, Asymptotics and special functions, AKP Classics, A K Peters Ltd., Wellesley, MA, 1997, Reprint of the 1974 original [Academic Press, New York; MR0435697 (55 \#8655)]. MR 1429619 (97i:41001)

[Paq82] L. Paquet, Problèmes mixtes pour le système de Maxwell, Ann. Fac. Sci. Toulouse Math. (5) 4 (1982), no. 2, 103-141. MR 687546 (84e:58075)

[RASi71] D. B. RAY and I. M. SINGER, R-torsion and the Laplacian on Riemannian manifolds, Advances in Math. 7 (1971), 145-210. MR 0295381 (45 \#4447)

[Spr05] M. SpreAfico, Zeta function and regularized determinant on a disc and on a cone, J. Geom. Phys. 54 (2005), no. 3, 355-371. MR 2139088 (2005k:11184)

[Spr06] M. Spreafico, Zeta invariants for Dirichlet series, Pacific J. Math. 224 (2006), no. 1, 185-200. MR 2231657 (2007b:11129)

[Ver09A] B. Vertman, Analytic torsion of a bounded generalized cone, Comm. Math. Phys. 290 (2009), no. 3, 813-860. MR 2525641 (2010d:58032)

[VER09B] Z Z Z Z _ determinants for regular-singular Laplace-type operators, J. Math. Phys. 50 (2009), no. 8, 083515, 23. MR 2554443

[VER11] The metric anomaly of analytic torsion at the boundary of an even dimensional cone, Ann. Global Anal. Geom. published online (2011), 1-30.

[Vis95] S. VishiK, Generalized Ray-Singer conjecture i: a manifold with a smooth boundary, Comm. Math. Phys. 167 (1995), 1-102.

[Wei80] J. Weidmann, Linear operators in Hilbert spaces, Graduate Texts in Mathematics, vol. 68, Springer-Verlag, New York, 1980, Translated from the German by Joseph Szücs. MR 566954 (81e:47001)

\section{List OF FIGURES}

1 Heat-space Blowup $\mathscr{M}_{h}^{2}$. 11

2 The lifted diagonal $\mathscr{D}$ of the heat-space $\mathscr{M}_{h}^{2}$. 15 
3 The deformed integration contour $\mathscr{C}_{\delta}$.

Mathematisches Institut, Universität Bonn, 53115 Bonn, Germany

E-mail address: mueller@math.uni-bonn.de

$U R L$ : www .math.uni-bonn.de/people/mueller

Mathematisches Institut, Universität Bonn, 53115 Bonn, Germany

E-mail address: vertman@math.uni-bonn.de

$U R L$ : www.math.uni-bonn.de/people/vertman 